\title{
Persée
}

http://www.persee.fr

\section{Le cheval du gisement pléistocène moyen de Camp-de-Peyre (Sauveterre-la-Lémance, Lot-et-Garonne)}

\author{
Jean-Luc Guadelli;François Prat \\ Paléo, Année 1995, Volume 7, Numéro 1 \\ p. $85-121$
}

Voir l'article en ligne

La faille ossifère découverte à Camp-de-Peyre en 1976 a livré les vestiges d'une faune froide du Pléistocène moyen, probablement mindélienne, dont l'un des éléments les plus représentatifs, avec le Renne, le Mouflon, le Lemming à collier et le Campagnol des hauteurs est un Equidé à face longue et museau court, aux formes élancées atteignant 1 , 50 à $1,55 \mathrm{~m}$ de haut au niveau du garrot. Cet animal associe des caractères primitifs à des dispositions typiquement caballines prédominantes. L'accent est mis sur les caractères odontologiques et ostéologiques qui distinguent les caballins ayant vécu antérieurement aux derniers épisodes nssiens des chevaux fossiles plus récents.

\begin{abstract}
Avertissement
L'éditeur du site «PERSEE » - le Ministère de la jeunesse, de l'éducation nationale et de la recherche, Direction de l'enseignement supérieur, Sous-direction des bibliothèques et de la documentation - détient la propriété intellectuelle et les droits d'exploitation. A ce titre il est titulaire des droits d'auteur et du droit sui generis du producteur de bases de données sur ce site conformément à la loi n`98-536 du 1 er juillet 1998 relative aux bases de données.
\end{abstract}

Les oeuvres reproduites sur le site «PERSEE » sont protégées par les dispositions générales du Code de la propriété intellectuelle.

Droits et devoirs des utilisateurs

Pour un usage strictement privé, la simple reproduction du contenu de ce site est libre.

Pour un usage scientifique ou pédagogique, à des fins de recherches, d'enseignement ou de communication excluant toute exploitation commerciale, la reproduction et la communication au public du contenu de ce site sont autorisées, sous réserve que celles-ci servent d'illustration, ne soient pas substantielles et ne soient pas expressément limitées (plans ou photographies). La mention Le Ministère de la jeunesse, de l'éducation nationale et de la recherche, Direction de l'enseignement supérieur, Sous-direction des bibliothèques et de la documentation sur chaque reproduction tirée du site est obligatoire ainsi que le nom de la revue et- lorsqu'ils sont indiqués - le nom de l'auteur et la référence du document reproduit.

Toute autre reproduction ou communication au public, intégrale ou substantielle du contenu de ce site, par quelque procédé que ce soit, de l'éditeur original de l'oeuvre, de l'auteur et de ses ayants droit.

La reproduction et l'exploitation des photographies et des plans, y compris à des fins commerciales, doivent être autorisés par l'éditeur du site, Le Ministère de la jeunesse, de l'éducation nationale et de la recherche, Direction de l'enseignement supérieur, Sous-direction des bibliothèques et de la documentation (voir http://www.sup.adc.education.fr/bib/). La source et les crédits devront toujours être mentionnés. 


\title{
LE CHEVAL DU GISEMENT PLEISTOCENE MOYEN DE CAMP-DE-PEYRE (Sauveterre la Lémance, Lot-et-Garonne) : Equus mosbachensis campdepeyri nov.ssp.
}

\author{
J.-L. GUADELLI ${ }^{(1)}$ et F. PRAT (2)
}

Résumé : La faille ossifère découverte à Camp-de-Peyre en 1976 a livré les vestiges d'une faune froide du Pléistocène moyen, probablement mindélienne, dont l'un des éléments les plus représentatifs, avec le Renne, le Mouflon, le Lemming à collier et le Campagnol des hauteurs est un Equidé à face longue et museau court, aux formes élancées atteignant 1,50 à 1,55m de haut au niveau du garrot. Cet animal associe des caractères primitifs à des dispositions typiquement caballines prédominantes. L'accent est mis sur les caractères odontologiques et ostéologiques qui distinguent les caballins ayant vécu antérieurement aux derniers épisodes rissiens des chevaux fossiles plus récents.

Mots-clés : Camp-de-Peyre, Pléistocène moyen, Mindel, Cheval, Equus mosbachensis.

Abstract : The ossiferous rift found at Camp-de-Peyre in 1976 gave remains of a cold fauna from the Middle Pleistocene period, probably Mindelian period. One of the most signifiant data is the presence with Reindeer, Moufflon, Collared Lemming and High mountain Vole of an Equidae with long face and short snout with slenderly measuring between $1 \mathrm{~m} 50$ and $1 \mathrm{~m} 55$ at the withers. This animal associats some primitive characteristics with some typically "caballine" dispositions. Emphasis is on odontologic and osteologic characters that make the distinction between horses which lived before the late rissian periods and more recent fossil horses.

Key-words : Camp-de-Peyre, Middle pleistocene, Mindel, Horse, Equus mosbachensis

\section{I) LE GISEMENT DE CAMP.DE-PEYRE.}

En 1976, la découverte de la faune pléistocène de Camp-de-Peyre pouvait être considérée, dans le domaine de la Préhistoire, comme un petit événement. Pour la première fois, en effet, elle révélait l'existence en France, antérieurement au Riss III, d'une association de mammifères caractérisée par la prédominance du Renne dont les restes, fait également insolite, voisinaient avec ceux d'un Mouflon de grande taille. Certes la présence de ces deux Herbivores avait déjà été signalée dans des dépôts jugés relativement anciens, plus précisément anté-rissiens : celle du Renne dans les alluvions vosgiennes d'Hangenbieten-Achenheim (Wernert, 1957), celle du Mouflon (Ovis antiqua) dans la haute terrasse de l'Allier à Pont-du-Château (Pommerol, 1879 et 1881 ; Rudel, 1953). Cependant, à
Camp-de-Peyre, Renne et Mouflon se trouvaient réunis au sein d'un ensemble comprenant d'autres Ongulés, des Carnivores, des Lagomorphes et des Rongeurs.

On sait maintenant que le gisement lot-etgaronnais ne constitue pas un cas unique puisque le Pléistocène moyen de la Caune de l'Arago à Tautavel (Pyrénées orientales) offre une association comparable (Crégut, 1979).

C'est le remplissage d'une des failles affectant les formations du Crétacé supérieur affleurant à Sauveterre-la-Lémance (Fig.1) qui recelait les ossements fossiles. Mis au jour lors des travaux d'exploitation du calcaire, il ne put malheureusement faire l'objet de fouilles systématiques. Néanmoins, les récoltes de sauvetage, ellesmêmes difficiles, furent effectuées par l'Institut du Quaternaire de l'Université Bordeaux I et la Direction des Antiquités Préhistoriques 
d'Aquitaine, dans la mesure du possible, en relation avec la stratigraphie établie par J.-M. Le Tensorer (Fig.2), auteur de l'étude géologique du site et des dépôts ayant comblé la fissure (Le Tensorer, 1973, 1981).

Ensemble supérieur : A-niveaux concrétionnés argileux, B-éboulis calcaires argileux, Cargiles sableuses rougeâtres, D-éboulis anguleux.

Ensemble moyen : 1-plancher stalagmitique, 2 et 3 -ensemble sableux, localement plus argileux, riche en faune, notamment en restes de chevaux, 4-complexe stalagmitique, 5-niveaux argilo-sableux rubéfiés, riches en faune mais pauvre en vestiges de chevaux, 6-niveau concrétionné, 7-argiles grises, 8-sables argileux roux fossilifères. Les couches 5 à 8 s'amincissent du Sud au Nord et disparaissent dans la petite salle.

Ensemble inférieur: Argiles versicolores avec passées sableuses.

A la publication en 1978 d'une première liste de taxons (Delpech et al) firent suite les précisions apportées par E. Donard (1982) sur les vestiges de Léporidés et les observations de J.-Cl. Marquet (1993) concernant les petits rongeurs.

\section{Genres et espèces identifiés :}

Mammifères à l'exclusion des Rongeurs (Delpech et al, 1978) :

- Couches 1 à 3 au-dessus du plancher 4 : Panthera sp. Oken, 1816

Meles sp. Brisson, 1762

Mustelidae Swainson, 1835 (plus petit que le Putois actuel mais plus grand que l'Hermine). Canis etruscus Forsyth Major, 1877.

“Cervus» s.l. Linné, 1758 (plutôt petit. Plusieurs individus).

Rangifer sp. H.Smith, 1827 (abondant). Bison sp. H.Smith, 1827 (plusieurs individus).

Ovis sp. Linné, 1758 (grande taille. assez abondant).

Equus caballus mosbachensis V. Reichenau, 1901.

Lepus timidus campdepeyri Donard, 1982 (couche 3).

Ochotona pusilla Pallas, 1769 (couche 3 in

Marquet, 1993).

- Couches inférieures sous le plancher 4 : Rangifer sp. H. Smith, 1827; couches 5 et 8 Bison sp. H.Smith, 1827 ; couches 5 et 8 Ovis sp. Linné, 1758. (grande taille); couches 5 et 8

Equus caballus mosbachensis V. Reichenau, 1901 ; couche 5

Lepus timidus campdepeyri Donard, 1982 ; couche 8
- Hors stratigraphie :

Alopex sp. Kaup, 1829.

Vulpes? Oken, 1816.

Ursus sp. Linné, 1758.

Rupicapra Frisch, 1775 / de Blainville, 1816.

Lepus timidus campdepeyri Donard, 1982.

Marmota Blumenbach, 1779.

Rongeurs (Marquet, loc. cit., p.95) :

Dicrostonyx torquatus Pallas, 1778

(couche 3).

Eliomys quercinus Linné, 1766 (couche 3).

Apodemus sylvaticus Linné, 1758

(couche 3).

Pitymys subterraneus de Sélys-Longchamps,

1836

(couches 3 et 8).

Arvicola terrestris Linné, 1758

(couche 3).

Microtus arvalis Pallas, 1778

(couche 3).

Allocricetus bursae Schaub, 1930

(couche 3).

Microtus gregalis Pallas, 1778

(couches 3 et 8 ).

Clethrionomys glareolus Schreber, 1780

(H.Strat.).

Microtus agrestris Linné, 1791 (H.Strat.).

Citellus superciliosus Kaup, 1839 (H.Strat.).

Pliomys lenki Heller, 1930 (H.Strat.).

Les différentes couches fossilifères présentant d'indéniables analogies faunistiques il serait déraisonnable de ne tenir aucun compte des pièces recueillies hors stratigraphie.

La faune de Camp-de-Peyre évoque un milieu ouvert sous la dépendance de conditions climatiques froides. La prédominance du Renne, la présence du Lemming à collier (Dicrostonyx torquatus) et du Campagnol des hauteurs (Microtus gregalis) laisse planer peu de doutes à cet égard.

Ce point de vue s'accorde bien avec les conclusions de l'étude sédimentologique. Pour J.M. Le Tensorer (loc. cit., p.120) la mise en place des couches fossilifères s'est effectuée au cours de périodes de rhexistasie.

- ensemble 6 à 8 : climat instable froid,

- ensemble 2 - 3 : climat d'abord modérément froid devenant de plus en plus rigoureux.

Toutefois J.-Cl. Marquet (1993, p. 96) note que dans ce contexte des espèces forestières ou dites tempérées, comme le Mulot sylvestre (Apodemus sylvaticus) et le Campagnol roussâtre (Clethrionomys glareolus), se sont maintenues ce qui suppose l'existence de lieux bien abrités où croissait une végétation arbustive.

Dans le premier article consacré au gisement ossifère de Camp-de-Peyre (Delpech et al, 1978) l'accent est mis sur la présence d'un Canis moins 
grand que les Loups würmiens et rissiens et le Loup de l'avant-dernier interglaciaire, Canis lupus lunellensis M.-F. Bonifay, 1971. II est hautement probable que le Canidé fossile de Sauveterre-laLémance était proche de celui, mindélien, de la grotte de l'Escale à Saint-Estève-de-Janson, bien connu par les travaux de M.-F. Bonifay (1968, 1971), et de celui de Nauterie à La Romieu (Gers) attribué à Canis etruscus cf. mosbachensis Soergel, 1925 (Prat et Thibault, 1976).

Selon J.-Cl. Marquet (loc. cit. p. 97), Allocricetus bursae duraciensis, déjà signalé à l'Escale, indique un âge Pléistocène moyen ancien tandis que les individus du genre Pitymys militent dans le sens d'une ancienneté du remplissage relativement grande. Nous tenons donc pour envisageable, voire assez vraisemblable, l'hypothèse selon laquelle la faune de Camp-dePeyre témoignerait d'une période froide antérieure à l'interglaciaire Mindel/Riss tout en restant conscients que les rares gisements du Pléistocène moyen connus nous renseignent mal sur la répartition dans le temps et l'espace de nos marqueurs chronologiques.

A Camp-de-Peyre, l'accumulation d'ossements pour la plupart entiers (sauf les plus fragiles), parfois en connexion anatomique, ne doit semble-t-il être imputée à une intervention humaine. Aucun document archéologique n'a d'ailleurs été découvert. II est facile d'imaginer qu'à un moment du Pléistocène moyen la faille, alors béante, joua le rôle de piège naturel. Par exemple, des animaux, presque toujours des Herbivores (qui vivent souvent en groupe), dans leur fuite éperdue devant un prédateur ou tout autre danger, ne purent éviter la chute dans le précipice.

\section{II) Le CheVal de CaMp-De-Peyre.}

II-1) Position stratigraphique des ossements de Chevaux (Fig.2, Tab.26).

Les ossements de Chevaux découverts au cours des fouilles de sauvetage appartenaient pour la plupart à l'ensemble moyen (couches 1 à 8). Ils se trouvaient en forte majorité dans la couche 3 limono-argilo-sableuse et, plus rarement, soit dans la couche argilo-sableuse 2 (pas toujours bien distincte de la précédente) soit à la fois inclus dans cette strate et le plancher stalagmitique 1. Une portion distale de tibia et un grand sésamoïde ont été dégagés des argiles 5 sousjacentes au plancher stalagmitique 4 de la salle du Cheval.

D'autre part, nous considérons comme très probablement contemporains de ceux de l'ensemble moyen plusieurs os et dents difficilement récoltés au pied de la face Nord du cône d'éboulis colmatant la fissure, ainsi que les documents retirés des déblais sur lesquels adhéraient sou- vent des sédiments apparemment identiques aux sédiments des couches 2 et 3 de la salle du Cheval et de la Petite salle situées respectivement au Sud et au Nord du cône d'éboulis.

Un des documents les plus remarquables est sans conteste une tête osseuse presque complète. Le crâne proprement dit auquel reste fixé, de chaque côté et en connexion anatomique, le sommet de la branche montante mandibulaire, gisait au sommet du remplissage de la salle du Cheval qui lui doit son nom. R. Pinéda le découvrit, le palais dirigé vers le haut, légèrement engagé dans la couche 2 et envahi par la calcite déposée lors de la formation du plancher stalagmitique 1. De fortes concrétions recouvrent encore la boîte crânienne tandis que les os de la face avaient été, à peu près, préservés de l'encroûtement. La région rostrale des prémaxillaires avec leurs incisives et l'essentiel de la mâchoire inférieure ont été recueillis plus tard, brisés. Nous dirons ultérieurement comment les relations ont été rétablies entre les trois pièces.

\section{II-2) La tête osseuse.}

II-2-1) Le crâne proprement dit (PI.I).

II-2-1-1) Etat de conservation.

La partie correspondant au museau, trouvée à part et récemment reconstituée, comporte la canine droite et les 6 incisives maintenues en place par les prémaxillaires dont il ne subsiste guère que la face caudale. Elle n'offre néanmoins aucun point de contact avec le calvarium dégagé par $R$. Pineda.

Norma verticalis (Vue frontale)

C'est elle qui met le mieux en évidence les dégâts subis par le calvarium (PI.I et Fig.3).

La majeure partie des os nasaux située en arrière du bord antérieur des orbites a été détruite de même que leur processus rostral. L'angle de l'incisure naso-incisive reste toutefois repérable du côté gauche. A ce niveau un petit bloc de sédiments durcis bouche incomplètement l'ouverture nasale.

Des os frontaux il ne reste que les processus zygomatiques et une zone relativement étroite (surtout du côté droit) longeant le bord supérieur de chaque orbite. Les pariétaux eux-mêmes ont été défoncés, essentiellement dans leur région antéro-médiane, plus faiblement en arrière des arcades zygomatiques de part et d'autre de la ligne de suture (non visible) des deux os.

Vu de dessus le crâne apparaît donc largement ouvert vers le haut et l'on découvre ainsi les vestiges encroûtés de l'ethmoïde.

Norma lateralis (vues latérales droite et gauche)

Les côtés, gauche et droit, ne diffèrent pas sensiblement en ce qui concerne leur état de conservation. Tous deux montrent un museau 
incomplet et un profil fâcheusement interrompu en raison de la détérioration des os nasaux, frontaux et pariétaux (PI.I et Fig.4).

En arrière des $\mathrm{P}^{2}$, la face latérale des maxillaires se révèle intacte, de même que l'os jugal, le processus zygomatique du frontal et celui du temporal. Leur encroûtement quasi inexistant prend, par endroits, la forme d'une fine pellicule qui n'oblitère guère les détails anatomiques.

En revanche, les dépôts de calcite sont particulièrement considérables dans la région temporo-occipitale où, d'autre part, des sédiments bréchifiés maintiennent fermement en connexion avec le calvarium chacune des deux portions dorsales mandibulaires conservées.

Norma basilaris (Vue ventrale)

Le bord caudal de l'ouverture des choanes et toute la zone située en arrière de celle-ci, à l'exclusion des maxillaires supérieurs et des arcades zygomatiques, disparaissent sous une profusion de bourgeons de calcite. La face occlusale des prémolaires et molaires, toutes présentes, libérée d'une gangue coriace autorise maintenant l'examen des replis de l'émail. Enfin, si une forte épaisseur de dépôts bréchifiés recouvre la région palatine des maxillaires supérieurs, les palatins ne sont que très peu encroûtés : le bord rostral de l'ouverture des choanes peut donc être reconstitué avec une précision tout à fait satisfaisante. L'emplacement du basion reste également bien déterminable.

Norma occipitalis (Vue caudale)

Rien à signaler si ce n'est le concrétionnement généralisé de la face caudale du crâne et le fait que le processus jugulaire et le condyle gauche de l'occipital ont été endommagés avant la formation du plancher stalagmitique.

\section{II-2-1-2) Caractères non mesurables remar- quables}

Le travail de reconstitution de la mâchoire inférieure et de la région incisive des prémaxillaires a rendu possible le rétablissement de connexions entre le crâne proprement dit et la mandibule. II devait aboutir à une mise en continuité de la partie de la mâchoire inférieure restée indépendante et de son sommet maintenu en articulation avec l'os temporal. De plus, les sédiments bréchifiés, qui au départ recouvraient la face occlusale de la 2ème prémolaire supérieure droite, portaient encore l'empreinte de la 2ème prémolaire inférieure droite. D'autre part, l'extrémité libre des 12 et I3 supérieures gauches se logeait de façon parfaitement adéquate dans l'intaille constituée par la calcite qui adhérait au sommet des incisives inférieures.

Notons aussi que le degré d'usure de chaque dent s'harmonise avec celui des autres compte tenu des dates d'éruption.

Norma lateralis (PI.I et Fig.4)
Au-dessous de la partie conservée de l'os incisif gauche, à environ 5 à $6 \mathrm{~cm}$ en avant de l'incisure naso-incisive, au-dessus de $\mathrm{P}^{2}$, le maxillaire est déprimé mais cette disposition résulte, du moins en partie, d'un enfoncement accidentel dont témoignent des lignes de fissure. On note aussi l'existence d'une dépression entre l'extrémité caudale du processus nasal de l'os incisif et le point de rencontre de l'os nasal, du maxillaire et du lacrymal.

Comparée à celle des crânes de chevaux dont nous disposons pour comparaison, la crête faciale, surtout bien conservée du côté gauche, n'est que faiblement proéminente mais il convient de rappeler que nous avons affaire à un sujet relativement jeune. Plus surprenant est le renflement grosso modo fusiforme qui affecte sa face ventrale à l'aplomb des arrières-molaires. Nous ignorons encore s'il est ou non de nature pathologique.

Au-dessus de la crête faciale le maxillaire supérieur est relativement peu déprimé tandis que l'os jugal se révèle modérément mais nettement bombé.

Du côté droit l'emplacement du tubercule facial ne peut être déterminé mais celui de son homologue gauche se situe en regard du parastyle de $\mathrm{M}^{1}$.

Entre le point de jonction de l'os nasal, du maxillaire et du lacrymal d'une part et le point de contact du maxillaire, du lacrymal et du jugal d'autre part, on observe une légère proéminence bulbeuse. Plus discrète sur la face latérale droite que sur la gauche, elle apparaît assez bien circonscrite alors que sur les crânes de chevaux actuels, dans la zone correspondante, un renflement est à peine perceptible.

Le processus lacrymal fait défaut ou, plus exactement, là où l'on s'attend à le trouver n'existe qu'un petit cercle rugueux circulaire. L'examen n'a pas permis de déterminer si le dit processus a été brisé

Nous ne possédons aucun élément permettant la reconstitution du processus rostral des os nasaux mais, plus en arrière, ceux-ci apparaissent légèrement concaves tandis qu'au dessus de l'orbite la partie conservée de l'os frontal gauche se dresse vers le plan médian de façon sensible, ce qui n'est pas le cas chez la plupart des chevaux actuels. Nous sommes donc fondés à penser que les os frontaux étaient surélevés et qu'à une ensellure nasale faisait suite caudalement une convexité frontale, caractères assez fréquents chez les Equidés du Pléistocène ancien et certains Zèbres. A cet égard notre spécimen diffère du crâne de Mosbach étudié par W. von Reichenau (1915) et de celui de Lunel-Viel dont M.-F. Bonifay (1980) nous dit qu'il possède un profil pratiquement rectiligne «-sans trace de bombement frontal-». D'autre part sur le crâne de 
Camp-de-Peyre, en avant de la protubérance occipitale externe, le profil de la crête sagittale externe se caractérise par une concavité particulièrement accentuée, ce qui devait contribuer à rendre encore plus sensible le caractère précédemment indiqué.

L'inion n'est pas repérable avec précision en raison de quelques dommages ayant affecté la région nucale mais la ligne joignant son emplacement présumé au tubercule facial passe au-dessous de l'orbite, trait peu commun chez Equus caballus. Nous ne sommes pas autorisés toutefois à conclure à une réelle flexion du crâne cérébral par rapport à la face. Selon V. Gromova $(1949 ;$ p.20) la verticale menée du bord rostral de l'orbite rencontre souvent le maxillaire supérieur à l'aplomb du métastyle de $\mathrm{M}^{3}$ chez Equus caballus et en arrière de cette dent chez Equus stenonis. Cette dernière disposition se retrouve sur le crâne de Camp-de-Peyre et d'après M.-F. Bonifay (loc cit) sur le crâne de Lunel-Viel, ce que montre la figure 5 publiée par cet auteur.

Norma verticalis (PI.I et Fig.3)

L'os nasal gauche dans sa partie rostrale conservée et l'os nasal droit, plus endommagé, présentent, juste en avant du creux de l'ensellure, une convexité modérée mais nette dans le sens transversal. Une légère gouttière parcourait la suture nasale au moins sur une certaine longueur. Une gouttière internasale existe aussi chez l'Equus de Lunel-Viel (Bonifay, 1980).

\section{II-2-1-3) Caractères mesurables}

Leur mise en évidence s'appuie sur des comparaisons effectuées entre le crâne de Camp-dePeyre et celui d'autres Chevaux pléistocènes et holocènes.

Dans le tableau ci-après figurent la provenance et le nombre de spécimens mesurés avec l'indication chronologique correspondante ainsi que le nom des auteurs auxquels nous nous référons. Nous remercions vivement Messieurs P. Caillat et J.-F. Tournepiche qui nous ont permis d'utiliser des données inédites.

Les diagrammes élaborés suivant la méthode de G. Simpson (1941) comportent de larges emprunts à la publication de V. Eisenmann, E. Crégut et A.-M. Moigne (1985) traitant des grands chevaux pléistocènes. L'emplacement des points déterminant le tracé de la courbe correspond à la différence entre le logarithme de chaque mesure prise sur le crâne considéré (ou sa valeur moyenne quand il s'agit d'une série de spécimens) et celui de la dimension homologue chez l'Hémione (Equus hemionus onager), Equidé ayant déjà été choisi comme référence par V. Eisenmann et al (loc. cit.). Les mesures retenues comptent parmi celles qui, d'après les auteurs précités, donnent pour le genre Equus les meilleures indications concernant la structure crânienne et la significa- tion des dissemblances observées entre divers groupes.

Les dimensions retenues sont les suivantes (Fig.5) : 1-Longueur basilaire, 2-Longueur palatine, 4-Longueur Vomer/Basion, 5-Longueur du museau, "2-5"-Longueur du palais, 6-Longueur du diastème, 7-Diamètre mésio-distal $\mathrm{P}^{2}-\mathrm{P}^{4}$, 8Diamètre mésio-distal $\mathrm{P}^{2}-\mathrm{M}^{3}$, 13-Largeur frontale, 15-Largeur maximum de la boîte crânienne, 16Largeur maximum de la protubérance occipitale externe, 17-Largeur du prémaxillaire, 17bLargeur minimum du museau, 21 Diamètre antéro-postérieur de l'orbite, 23-Longueur de la ligne oculaire antérieure, 24-Longueur de la ligne oculaire postérieure, 25-Hauteur de la face en avant de $P^{2}, 28$-Hauteur de la face en arrière des orbites.

\section{Figures 6 et 8}

Comparativement aux crânes de chevaux actuels pris en compte lors de l'élaboration du diagramme correspondant à la figure 8 , le spécimen de Camp-de-Peyre (Fig.6) se caractérise par:

- un museau proportionnellement plus court (mesure 5) et vraisemblablement plus large dans la région de la barre si l'on en juge d'après le rétrécissement correspondant de la mâchoire inférieure.

- un palais plus allongé (mesure 2-5).

- un développement plus grand de la partie située en avant du bord postérieur des orbites comme l'indique la longueur de la ligne oculaire antérieure (mesure 23).

\begin{tabular}{|c|c|c|c|}
\hline & $\mathrm{n}$ & & \\
\hline $\begin{array}{l}\text { Mosbach (Rhénanie. } \\
\text { Allemagne) }\end{array}$ & 2 & $\begin{array}{l}\text { Pléistocène } \\
\text { moyen }\end{array}$ & $\begin{array}{l}\text { von Reichenau. 1915: V } \\
\text { Gromova. 1949. t.1 : V } \\
\text { Eisenmann et al. 1985 }\end{array}$ \\
\hline I.unel-Viel (Hérault) & 1 & $\begin{array}{l}\text { Pléistocène } \\
\text { moyen }\end{array}$ & $\begin{array}{l}\text { M.- I. Bonifay, } 1980: \mathrm{V} \text {. } \\
\text { Eisenmann et al., } 1985\end{array}$ \\
\hline $\begin{array}{l}\text { La Caune de l'Arago } \\
\text { (Pyrénées-orientales) }\end{array}$ & 16 & $\begin{array}{l}\text { Pléistocène } \\
\text { moyen }\end{array}$ & $\begin{array}{l}\text { E. Crégut, 1979; V } \\
\text { lisenmann et al. 1985 }\end{array}$ \\
\hline Siréjol & 2 & Würm récent & $\begin{array}{l}\text { J.-F. Tournepiche (données } \\
\text { inédites) }\end{array}$ \\
\hline I.e (Quéroy (( harentes) & 2 & Würm récent & $\begin{array}{l}\text { J.-F. Tournepiche (donnees } \\
\text { inédites) }\end{array}$ \\
\hline Saintes (Charentes) & 46 & $\begin{array}{l}\text { Holocène } \\
\text { (iallo-Romain }\end{array}$ & $\begin{array}{l}\text { P. Caillat (1994 et donnéce } \\
\text { inédites) }\end{array}$ \\
\hline ( heval de Przeu'alsky & 20 & Holocène & V. Ëisenmann. 1980 \\
\hline (Chevaux Lourds & 29 & Holocène & V. Eisenmann, 1980 \\
\hline Pottock actuels & 4 & Holocène & $\begin{array}{l}\text { Collection de comparaison. } \\
\text { Institut du Quaternaire, }\end{array}$ \\
\hline
\end{tabular}

- une largeur frontale (mesure 13) plutôt faible, comme celles des crânes du site galloromain de Saintes.

- des orbites plus modérément ouvertes dans le sens antéro-postérieur (mesure 21).

Figures 6,7 et 8

Ainsi que l'on pouvait s'y attendre, les crânes würmiens du Quéroy et de Siréjol (Charente) ont une longueur basale inférieure à celle du crâne de Camp-de-Peyre (Fig.6 et 7). Ils possèdent un 
palais relativement plus long que celui des Chevaux holocènes auxquels nous les comparons tandis que leur museau est à la fois plus court et plus large (Fig.7 et 8). En outre si l'on considère la largeur minimum du museau entre les dents frontales et les $\mathrm{P}^{2}$ (mesure 17b) on voit qu'elle surpasse très nettement celle des crânes actuels et sub-actuels. Les mesures 25 et 28 qui expriment respectivement la hauteur du crâne en arrière des orbites et en avant de $\mathrm{P}^{2}$ (non prises sur le crâne de Camp-de-Peyre) fournissent des chiffres supérieurs à ceux relevés chez Equus przewalskii et les chevaux domestiqués galloromains de Saintes.

\section{Figure 6}

Nous sommes naturellement amenés à comparer entre eux les crânes plus ou moins bien conservés découverts dans les gisements du Pléistocène moyen. II y en aurait 4 au total en ce qui concerne l'Europe occidentale dont 2 trouvés en France. II faut cependant y ajouter, pour ce dernier pays, de belles portions crâniennes provenant de la Caune de l'Arago. C'est donc peu de dire que l'effectif est très modeste alors que la période concernée s'évalue en centaines de milliers d'années !! Nous voilà tenus à proscrire ici toute conclusion de portée trop générale.

- le crâne de Camp-de-Peyre, un peu plus grand que celui de Lunel-Viel, n'atteint pas les dimensions de celui de Mosbach (Tab.1).

- En regard de celui de la grotte de Lunel-Viel, le spécimen lot-et-garonnais possède un museau plus court (mais il se rapporte à un sujet encore relativement jeune), plus large et, d'après ce que l'on sait du rétrécissement de la mâchoire inférieure en arrière des incisives, moins étroit dans la région de la barre. Sa face paraît aussi relativement un peu moins longue, ainsi que le donne à penser la comparaison des lignes oculaires antérieures (mesure 23).

Quant aux restes crâniens recueillis à la Caune de l'Arago, ils indiqueraient par rapport à ce que l'on observe sur les crânes de Camp-dePeyre et de Lunel-Viel un palais plus allongé (mesure 2-5) et une cavité orbitaire particulièrement courte (mesure 21).

En ce qui concerne le crâne de Mosbach il diffère de ceux de Camp-de-Peyre, La Caune de l'Arago et Lunel-Viel par sa plus grande largeur frontale (mesure 13).

Enfin ces quelques spécimens du Pléistocène moyen présentent quelques traits communs (hormis leurs fortes dimensions) qui semblent les différencier des chevaux holocènes auxquels ils sont comparés :

- palais relativement plus long (mesure 2-5),

- diamètre transversal du prémaxillaire (mesure 17) plus important mis à part le crâne de Lunel-Viel,
- diamètre antéro-postérieur de l'orbite (mesure 21) plus court.

\section{II-2-2) La mandibule.}

La région incisive et les deux corps ont été presque intégralement reconstitués. La partie de la branche montante qui prolonge le corps mandibulaire droit, réduite ou peu s'en faut à son bord caudal, entre heureusement en contact avec la portion de la mâchoire inférieure restée en connexion anatomique avec le crâne proprement dit. Si le bord inférieur des corps mandibulaires en arrière de la symphyse est détruit entièrement (corps mandibulaire droit) ou partiellement (corps mandibulaire gauche) on constate que la région radiculaire des mâchelières est relativement bien conservée. Toutes les dents (incisives, canines, prémolaires et molaires) sont présentes sauf la $\mathrm{M}^{3}$ gauche.

Sa symphyse, mesurée du côté lingual, est relativement peu allongée : $89,3 \mathrm{~mm}$. En effet, sur les deux spécimens de Mosbach étudiés par Von Reichenau (1915) elle atteint respectivement 96 et $120 \mathrm{~mm}$ et sur la portion mandibulaire de l'Equidé mindélien de Montoussé (HauteGaronne) conservée au Muséum d'histoire naturelle de Bordeaux, 103mm. Parmi 11 mâchoires inférieures réduites à leur région rostrale ayant appartenu à des chevaux du Würm ancien de provenances diverses (Prat, 1968, tab.5) trois seulement ont une symphyse un peu plus courte $(86,87$ et $88,5 \mathrm{~mm})$. Sur sept des huit autres la longueur de la symphyse dépasse $95 \mathrm{~mm}$. Ces pièces qui représentent probablement des individus moins grands que ceux de Camp-dePeyre, se rapportent en majorité à des mâles $(n=8)$.

Notons enfin que la mâchoire inférieure découverte à Lunel-Viel possède une symphyse dont la longueur (92mm selon M.-F. Bonifay, 1980) est du même ordre de grandeur que celle de la mandibule de Camp-de-Peyre.

La largeur au niveau du rétrécissement mandibulaire, juste avant que ne se séparent les deux corps, ne peut être mesurée avec exactitude mais évaluée à $52 \mathrm{~mm}$. Le rétrécissement serait moins accusé que sur la mandibule de Lunel-Viel $(38,8 \mathrm{~mm})$.

L'arc des incisives se révèle plutôt étroit : $71,3 \mathrm{~mm}$. Chez les chevaux würmiens cette valeur est, semble-t-il, dépassée puisque dans 14 cas sur 18 la largeur de la série varie de 73 à $82,5 \mathrm{~mm}$ (Prat, 1968, tab.5).

Néanmoins, une autre série de 6 incisives trouvées également en connexion à Camp-dePeyre, légèrement plus usées que celles de la mandibule $n^{\circ} 234$ à une largeur de $82 \mathrm{~mm}$, ce qui témoigne d'une certaine ampleur des variations individuelles chez notre Equidé mindélien, du moins sous ce rapport. 
La portion $\mathrm{d}$ e mandibule gauche maintenue en contact avec le calvarium par des sédiments concrétionnés (Fig.4 et PI.I) est de dimensions réduites. Pourtant il paraît clair qu'au-dessous du condyle, en vue latérale, le bord caudal de la branche montante se dirige obliquement de haut en bas vers l'avant de telle sorte qu'il devait se situer, sur toute sa hauteur, en avant du plan vertical tangent au bord postérieur du condyle. Ce caractère sans doute assez fréquent chez Equus stenonis a été observé chez le cheval fossile, rissien ou anté-rissien, de Torralba en Espagne (Prat, 1977, p.34, pl.I). Chez les chevaux actuels le dit plan coupe souvent la branche montante mandibulaire (Gromova, 1949, p.19).

\section{II-2-3) Age et sexe de l'individu représenté}

Les incisives, prémolaires et molaires de 2ème dentition, toutes présentes sur l'arcade à l'exception de la M3 inférieure gauche, étaient entrées en activité et les canines dont la région cervicale ne se situe pas encore au niveau du bord alvéolaire n'avaient point encore pris tout à fait leur place définitive. L'existence de ces dents, leur stade d'éruption et leurs dimensions prouvent que nous avons affaire à un sujet mâle mort dans sa 5ème année (prenant 5 ans) si on se fie aux séquences d'éruption dentaire de A. Goubaux et G. Barrier (1884 et 1890).

\section{II-3) Les dents.}

II-3-1) Présence des dents de Loup D1 (P1).

Deux des 4 portions de maxillaires conservées possèdent une dent de loup ( $D^{1}$ ou $\mathrm{P}^{1}$ ). Celle du spécimen $n^{\circ} 150$ (c.3) qui porte une série $D^{1}\left(P^{1}\right)$ $D^{4}$ droite est malheureusement cassée au niveau du collet mais celle appartenant à la série $D^{1}\left(P^{1}\right)$ $D^{4}$ du maxillaire $n^{\circ} 146$ (c.2) est intacte : son diamètre mésio-distal égale $9,8 \mathrm{~mm}$ et son diamètre vestibulo-lingual $5,9 \mathrm{~mm}$ (PI. II)

D'autre part, la mandibule $\mathrm{n}^{\circ} 133$ (c.2) présente une petite $D_{1}$ (ou $P_{1}$ ) caniniforme visible dans son alvéole (PI. III). II semble que les pièces $n^{\circ} 146$ et 133 aient appartenu à un même sujet âgé d'environ un an.

Rappelons que M. Petit (1939) signale que dans une population homogène de Chevaux de race Barbe-arabe la proportion de $D^{1}\left(P^{1}\right)$ supérieures est de 1 sur 10 alors que celle de $D_{1}\left(P_{1}\right)$ inférieures est de 1 sur 1000.

II-3-2) Les dents définitives.

II-3-2-1) Incisives et Canines.

Parmi les 6 I1 sup., 7 I1 inf., 5 I2 sup., 6 I2 inf., 6 I 3 sup. et 4 I 3 inf. seules les 13 inf. qui appartiennent au crâne $n^{\circ} 234$ ont le cornet externe ouvert de même que les 11 sup. droite, I1 inf. droite et gauche de la série $n^{\circ} 252$.

(Fig.9).

II-3-2-2) Prémolaires et Molaires supérieures

\section{P2}

Le mésostyle des 7 spécimens est large et dédoublé. Le flanc vestibulaire de leur paracône est concave dans un cas, plan sur 3 dents et faiblement convexe dans les 3 autres cas. Le flanc vestibulaire du métacône est concave 5 fois sur 7 , plan 2 fois et convexe dans le dernier cas. Le pli caballin, présent 5 fois sur 7 , mesure entre 3 et $4 \mathrm{~mm}$. Quant à la face linguale du protocône elle présente un sillon longitudinal large et peu profond dans 5 cas alors qu'elle n'est affectée que d'une simple ondulation sur les 2 autres dents.

P3

Leur parastyle est large et dédoublé sur les 6 spécimens mais faiblement dédoublé sur deux d'entre eux. Le mésostyle, toujours large, se dédouble dans 4 cas. Notons que sur les $2 \mathrm{P}^{3}$ où ce style est simple il est également peu large. Si la face vestibulaire du paracône est chaque fois concave, celle du métacône se révèle concave dans 4 cas et plan dans le dernier cas observable (le métacône est endommagé sur une dent). Le pli caballin, présent, mesure entre 3 et $4 \mathrm{~mm}$. Le sillon longitudinal lingual du protocône est large et profond.

P4

Leur parastyle est large et dédoublé sur 6 spécimens mais faiblement dédoublé sur deux d'entre-eux. Le mésostyle est toujours large, dédoublé dans 4 cas et simple dans les 2 autres. Sur les $2 \mathrm{P}^{4}$ possédant un mésostyle simple, celui-ci est très modérément large. Le flanc vestibulaire du paracône est chaque fois concave de même que celui du métacône. Le pli caballin, toujours présent, mesure entre 3 et $4 \mathrm{~mm}$. Le flanc lingual du protocône montre un sillon longitudinal étroit et peu profond. Une $\mathrm{P}^{4}$ en est toutefois dépourvue.

M1

Le parastyle, étroit et simple dans 8 cas, est modérément large et faiblement dédoublé sur 2 dents. Le mésostyle, constamment étroit $(n=10)$, apparaît simple dans 7 cas et légèrement dédoublé sur les $4 / 5$ de sa hauteur sur 3 dents. Le flanc vestibulaire du paracône et du métacône est concave. Le pli caballin, absent 3 fois sur 6 , ne mesure que $1 \mathrm{~mm}$ sur les $3 \mathrm{M}^{1}$ où il est observable. Le flanc lingual du protocône présente un sillon longitudinal étroit $(n=4)$ ou large $(n=3)$, peu profond $(n=6)$ ou profond $(n=1)$. II affecte une forme légèrement ondulée dans deux cas et sur un des exemplaires il ne présente aucune inflexion.

M2

Sur 3 des 6 dents le parastyle est étroit et simple, sur deux autres il est modérément large et simple et sur la sixième il est large et dédoublé. Le mésostyle est étroit et simple sur 2 dents, étroit et simple avec une fine rainure dans 3 cas, étroit et dédoublé sur l'autre dent. La face vestibulaire du paracône est concave, celle du métacône aussi, sauf dans un cas où elle se montre plan convexe. Sur les 4 molaires se prêtant à l'ob- 
servation, le pli caballin, présent sur 3 dents, mesure de 1 à $3,5 \mathrm{~mm}$. Le flanc lingual du protocône présente un sillon longitudinal étroit et peu profond $(n=3)$, large et profond $(n=1)$ et réduit à 2 infléchissements de l'émail dans les 2 cas restants.

\section{M3}

6 de ces dents ont un parastyle étroit et simple. Quant au mésostyle il est étroit mais élargi vers le bas de la couronne dans 6 cas, rainuré $(n=1)$, franchement dédoublé $(n=1)$ ou simple $(n=4)$. Le flanc vestibulaire du paracône et celui du métacône sont concaves. Sur les 4 dents dont l'état de conservation autorise cette observation le pli caballin est présent dans 2 cas et mesure de 1 à $2 \mathrm{~mm}$. La postfossette est ouverte vers l'arrière dans un cas, fermée avec, distalement, un îlot d'émail dans 3 cas et fermée sans îlot d'émail dans 1 cas. Le flanc lingual du protocône possède un sillon longitudinal très étroit et peu profond $(n=1)$, étroit et profond $(n=1)$, large et très peu profond $(n=1)$, large et profond $(n=1)$, ondulé $(n=1)$. II n'est absent qu'une seule fois. Le métastyle dépasse systématiquement l'hypocône vers l'arrière. Enfin, en vue linguale ou vestibulaire la couronne apparaît chaque fois faiblement recourbée.

Le tableau 3 regroupent les dimensions de ces dents jugales.

Lorsqu'on compare l'évolution de l'indice protoconique dans la série des dents jugales supérieures des Chevaux du Pléistocène moyen (Camp-de-Peyre, Mosbach, Lunel-Viel, l'Arago, Montoussé, La Micoque, Vergranne) à ce qu'elle est chez les Chevaux plus récents (Riss III de l'abri Suard, Würm ancien de Combe-Grenal, interstade würmien de Camiac, Würm récent de Solutré (Périgordien et Magdalénien), Holocène, on constate qu'il existe une différence fondamentale entre les 2 lots (Fig.10, 11, 12, 13). De manière constante, on observe chez les premiers une progression de l'indice protoconique de $\mathrm{P}^{2}$ à $\mathrm{P}^{4}$ et un indice protoconique de $\mathrm{M}^{1}$ peu différent de celui de la $\mathrm{P}^{4}$ (Fig. 10). V. Eisenmann (1980, p.124) signale même au sujet des spécimens qui proviennent des sables de Mosbach, que "...dans $71 \%$ des cas, l'IP de la $M^{1}$ est plus petit que celui de la $P^{4}$ ». Enfin l'indice protoconique de $\mathrm{M}^{2}$ est le plus souvent inférieur à celui de $\mathrm{M}^{3}$.

$\mathrm{Si}$ on ne fait pas la distinction (parfois malaisée dans le cas de dents isolées) $\mathrm{P}^{3}-\mathrm{P}^{4}$ et $\mathrm{M}^{1}-\mathrm{M}^{2}$, on remarque aussi en ce qui concerne l'indice protoconique que celui des $\mathrm{P}^{3}-\mathrm{P}^{4}$ surpasse très nettement en moyenne celui des $\mathrm{P}^{2}$ et qu'il progresse plus faiblement des prémolaires à $\mathrm{M}^{3}$ (Fig.12). II arrive néanmoins, dans de rares cas, que l'indice protoconique de $\mathrm{M}^{3}$ soit un peu inférieur à celui des $\mathrm{M}^{1}-\mathrm{M}^{2}$. (Fig.12 $\mathrm{n}^{\circ} 2$ ). II n'est pas interdit de penser que ces exceptions à la «règle» sont imputables au fait qu'il est difficile, quand on mesure le diamètre mésio-distal du protocône et de la couronne des $\mathrm{M}^{3}$, d'éliminer les effets de la courbure de ces dents qui détermine l'obliquité accentuée de la face occlusale.

En revanche chez les chevaux ayant vécu en France à partir du Riss III, en l'état actuel de nos connaissances, l'indice protoconique progresse assez régulièrement de $\mathrm{P}^{2}$ à $\mathrm{M}^{2}$ alors qu'il est plus faible sur $\mathrm{M}^{3}$ que sur la deuxième molaire supérieure (Fig.11 et 13). Cette différence entre ces Equidés et les Chevaux plus anciens a d'ailleurs déjà été signalée (Guadelli et Prat, 1983; Guadelli, 1987, 1989). Les séries de Camp-de-Peyre ne font que confirmer cette observation.

Malheureusement, dans l'hypothèse où nos chevaux du Pléistocène supérieur dériveraient de ceux (ou de l'un de ceux) du Pléistocène moyen que nous avons évoqués, nous ignorons à quoi correspondent ces modifications dentaires et les causes qui les ont amenées.

Nous rappellerons cependant ici que l'un de nous (Guadelli, 1987 et 1990) a proposé une explication pour les variations de l'indice protoconique en fonction des changements de l'environnement au cours du Paléolithique. Ceux de ces animaux contraints à se nourrir de végétaux coriaces ou chargés de poussières abrasives (milieux steppiques) usaient plus vite leurs molaires que ceux qui disposaient d'aliments plus tendres. De même qu'une augmentation de la hauteur de la couronne, l'allongement du protocône, élément ayant pu varier dans une certaine mesure indépendamment du reste de la couronne, pouvait peut-être compenser les effets d'une usure particulièrement intense.

\section{II-3-2-2) Prémolaires et Molaires inférieures} (Fig.14)

P2

Sur ces dents, le sillon lingual de la doubleboucle est à peine indiqué, le sillon vestibulaire court et le pli ptychostylide large et long $(2 \mathrm{~mm})$. Le flanc vestibulaire du protoconide est plan $(n=2)$, convexe $(n=2)$ et celui de l'hypoconide concave $(n=6)$

P3

Les $4 \mathrm{P}_{3}$ ont une double boucle d'allure caballine avec un métaconide globuleux et un métastylide triangulaire séparés par un sillon lingual en $\mathrm{V}$ large à fond arrondi. Le sillon vestibulaire est court et le pli ptychostylide égal à $2 \mathrm{~mm}$ sur les 2 dents dont l'état de conservation rend l'observation possible. Le flanc vestibulaire du protoconide et celui de l'hypoconide sont concaves. Sur la $P_{3}$ gauche $n^{\circ} 166$ le préflexide est remplacé par un îlot d'émail.

P4

Elles possèdent toutes une double-boucle caballine avec un métaconide globuleux et un 
métastylide triangulaire. Le sillon lingual est en $U$ large à fond arrondi $(n=2)$ ou en $V$ ouvert à fond arrondi $(n=3)$. Sur les $3 P_{4}$ où l'observation est possible, le sillon vestibulaire est court et le pli ptychostylide mesure $2 \mathrm{~mm}(n=2)$ ou $3 \mathrm{~mm}(n=1)$. Le flanc vestibulaire du protoconide est concave de même que le flanc vestibulaire de l'hypoconide.

\section{M1}

Les 5 spécimens présentent une doubleboucle de type caballin avec un métaconide globuleux et un métastylide triangulaire. Le sillon lingual est en $U$ large à fond plat $(n=1)$, en $V$ ouvert à fond arrondi $(n=3)$ ou en $V$ ouvert à fond aigu $(n=1)$. Sur les $3 M_{1}$ se prêtant à l'observation le sillon vestibulaire est long : il pénètre dans le pédoncule de la double boucle sans entrer en contact avec le sillon lingual ; le pli ptychostylide se réduit à une ondulation dans 2 cas et mesure $1 \mathrm{~mm}$ sur l'autre $M_{1}$. Le flanc vestibulaire du protoconide est soit concave $(n=4)$, soit plan $(n=1)$ et celui de l'hypoconide chaque fois concave.

\section{M2}

Les 6 dents possèdent une double-boucle caballine avec un métaconide globuleux et un métastylide triangulaire. Le sillon lingual est en $U$ large à fond arrondi $(n=1)$ ou en $V$ ouvert à fond arrondi $(n=5)$. Parmi les 5 dents autorisant l'observation, le sillon vestibulaire est long ; il pénètre dans le pédoncule de la double boucle sans entrer en contact avec le sillon lingual. Le pli ptychostylide réduit à une ondulation dans 2 cas, mesure $1 \mathrm{~mm}$ dans 2 autres cas et $2 \mathrm{~mm}$ sur la cinquième dent. Le flanc vestibulaire du protoconide est concave $(n=4)$, plan $(n=1)$ ou légèrement convexe $(n=1)$, tandis que celui de l'hypoconide est concave.

M3

Les $7 M_{3}$ présentent une double-boucle caballine avec un métaconide globuleux et un métastylide triangulaire. Le sillon lingual est en $U$ large à fond arrondi $(n=1)$ ou en $V$ ouvert à fond arrondi $(n=6)$. Sur les $6 M_{3}$ où l'observation est possible le sillon vestibulaire est long ; il pénètre dans le pédoncule de la double boucle, sauf dans un cas, sans entrer en contact avec le sillon lingual. Le pli ptychostylide est réduit à une ondulation $(n=3)$, mesure $1 \mathrm{~mm}(n=1)$ ou $2 \mathrm{~mm}(n=2)$. Le flanc vestibulaire du protoconide est convexe $(n=5)$, concave $(n=1)$ ou plan $(n=1)$. Le flanc vestibulaire de l'hypoconide est plan $(n=4)$, concave $(n=1)$ ou convexe $(n=1)$. De manière générale le fût de la couronne est modérément courbe mais on constate en vue linguale (ou vestibulaire) que le bord distal décrit une forte concavité, surtout dans le cas de la molaire $n^{\circ} 127$ (Fig.14b et $14 c$ ).

Cette dernière disposition, peu fréquente chez les chevaux würmiens, liée à un développement de plus en plus accusé de l'hypoconulide du sommet à la base de la couronne, s'observe dans la plupart des cas chez Equus hydruntinus (Prat, 1968, p.554). Elle est également signalée chez Equus stenonis senezensis (Prat, loc. cit., p.95). S'agit-il de la persistance à Camp-de-Peyre d'un caractère archaïque ?

Les dimensions de ces dents regroupées tableau 7 n'appellent pas de commentaires.

II-4) Taille et silhouette du Cheval de Camp-dePeyre.

La plupart des éléments du squelette, souvent bien conservés, figurent dans la documentation dont nous disposons mais ils y sont inégalement représentés et chaque série de pièces homologues ne comporte, par rapport à quelques unes de celles auxquelles nous la comparons, qu'un nombre modeste de spécimens. Pourtant les chiffres fournis par les mensurations effectuées, notamment sur les os longs, autorisent parfois quelques conclusions.

En ce qui concerne les différences avec les chevaux würmiens, celles relatives à la taille sont évidentes : il suffit de comparer les dimensions des os entiers de Camp-de-Peyre (tableaux 12 à 25) aux dimensions correspondantes figurant dans les tableaux publiés par F. Prat (1968) ou J.L. Guadelli (1986, 1987, 1991). Nous nous bornerons donc à souligner ici que les métacarpiens III, 144 au total, découverts dans les gisements cités ci-après sont plus courts, à une exception près (un spécimen de Pair-non-Pair long de 242mm), que le moins grand des os canons de Camp-dePeyre (long $=238,5 \mathrm{~mm}$ ) (Fig.16 et $17 \mathrm{Tab} .17$ )

- Würm ancien : Combe-Grenal (Dordogne), Pair-non-Pair et Camiac (Gironde).

- Würm récent inférieur : Jaurens (Corrèze) d'après C. Mourer-Chauviré (1980), Solutré pro parte (Saône-et-Loire).

- Würm récent supérieur: Solutré pro parte (Saône-et-Loire) et Saint-Germain-la-Rivière (Gironde).

Les mensurations pratiquées sur les métatarsiens III des mêmes gisements, également très nombreux (112), donnent des résultats parfaitement concordants (Fig.18 et 19 et Tab.24).

Si l'on considère les valeurs de l'indice qui traduit la plus ou moins grande largeur des pattes dans la région des canons (indice de robustesse: largeur au milieu des métacarpiens ou métatarsiens III x 100 / longueur totale de l'os), celle pour laquelle nous possédons le plus de données significatives, les différences se révèlent moins accusées. II convient néanmoins de noter que les métacarpiens III, sauf un, et les métatarsiens III de Camp-de-Peyre sont proportionnellement moins larges en leur milieu que ne le sont en moyenne ceux des chevaux würmiens, plus "graciles" même que ceux d'Equus caballus arcelini Guadelli, 1990 du Würm récent supérieur (Fig.16 à 19 et tableaux 17 et 24). 
En France, les gisements rissiens jusqu'ici exploités n'ont que rarement livré des séries d'os longs d'Equidés aussi riches que celles provenant des sites würmiens que nous venons d'évoquer.

Les os canons recueillis par P. Wernert (1957) dans le loess ancien d'Achenheim (Bas-Rhin) constituent toutefois de bons lots de comparaison. La longueur de 8 métacarpiens III, qui varie de $243 \mathrm{~mm}$ à $264,5 \mathrm{~mm}$, égale en moyenne $250,7 \mathrm{~mm}$ (Prat, 1968, tab.60) tandis que celle des os correspondants de Camp-de-Peyre se situe soit très près $(240,244,248 \mathrm{~mm})$ de la longueur la plus faible enregistrées à Achenheim, soit au-dessous de cette valeur $(238,5 \mathrm{~mm})$. D'autre part la longueur moyenne de 10 métatarsiens III des loess anciens d'Alsace, $(299 \mathrm{~mm}$, loc cit, tab.97) n'est dépassée à Camp-de-Peyre que dans un seul cas (spécimen $n^{\circ} 5$ ) et les 4 radius de ce gisement se placent en deçà de l'intervalle de variation des 4 radius d'Achenheim dont le moins grand mesure plus de $365 \mathrm{~mm}$ (loc cit, tab.50). Tout ceci nous incite à penser que le Cheval du pléistocène moyen de Sauveterre-laLémance était de moindre stature.

D'autres documents d'un grand intérêt ont été trouvés dans la haute terrasse de l'Isère à Châtillon-Saint-Jean (Drôme). En 1962 C. Chauviré a publié, entre autres, 16 os canons postérieurs longs en moyenne de $286,26 \mathrm{~mm} \pm$ 3,86 (262-297mm), donc de même ordre de grandeur que les 6 métatarsiens III de Camp-dePeyre (moyenne $=288,8 \mathrm{~mm}$ ). Nous reviendrons ultérieurement sur ces pièces.

A signaler encore 3 métatarsiens III provenant de la grotte de Coupe-Gorge (couche 3 anté-würmienne) à Montmaurin (Haute-Garonne). Ils ont certainement appartenu à un grand Cheval puisqu'ils mesurent respectivement $286 \mathrm{~mm}, 292 \mathrm{~mm}$ et $306 \mathrm{~mm}$ de long (Guadelli, 1990, tab.55).

Des talus, calcanéums et phalanges, en effectifs réduits, mis au jour dans des stations préhistoriques du Sud-Ouest de la France, La Micoque et Pech de l'Azé (Dordogne), l'abri Suard à la Chaise (Charente) se rapportent à des animaux de stature comparable à celle d'autres Equidés rissiens cités précédemment (Prat, 1968). Malheureusement, comme il est de règle dans les gisements archéologiques, leurs grands os longs ne sont conservés qu'exceptionnellement.

Comparer le Cheval de Camp-de-Peyre aux Chevaux anté-rissiens qui, pense-t-on, lui seraient chronologiquement plus ou moins proches, offre un intérêt particulier. Cela a déjà été réalisé pour le crâne et la denture. Toutefois nous nous heurtons encore au fait qu'à Camp-dePeyre aucune série d'os homologues n'est assez nombreuse pour obtenir une bonne estimation de l'étendue du domaine de variation.

Lorsque nous comparons l'Equidé de LunelViel (Hérault) étudié par M.-F. Bonifay (1980), Equus mosbachensis palustris (3), à celui de Sauveterre-la-Lémance, sans oublier ce que nous avons dit à propos de la relative pauvreté de nos échantillons, nous prendrons en considération les points suivants :

- parmi les 11 radius de Lunel-Viel, 9 ont une longueur inférieure à celle du plus petit des radius de Camp-de-Peyre tandis qu'aucun des deux autres n'atteint la taille du plus grand spécimen de ce dernier gisement (Tab.14).

- la longueur de chaque métacarpien III de Camp-de-Peyre surpasse, parfois très nettement, la longueur moyenne des 22 os canons antérieurs de Lunel-Viel (niveaux inférieur et supérieur), ceux-ci se révélant presque toujours proportionnellement plus larges en leur milieu (Fig.16 et Tab.17).

- la longueur moyenne des métatarsiens III de Lunel n'atteint pas, il s'en faut de beaucoup, la longueur du moins grand métatarsien III de Camp-de-Peyre. Le diamètre antéro-postérieur articulaire proximal des métatarsiens III est plus fort sur le Cheval de Camp-de-Peyre que sur celui des gisements de Mosbach et de Lunel-Viel (Fig.18 et Tab.24).

Enfin on notera, à propos des canons postérieurs de Camp-de-Peyre, le fort développement de la facette articulaire qui répond au cuboïde ainsi que le diamètre antéro-postérieur maximum relativement élevé du condyle interne (Fig.17 et Tab.24).

Lorsqu'on porte son attention sur les autres éléments du squelette, on constate chaque fois une différence de taille à l'avantage (si l'on peut dire) des pièces de Camp-de-Peyre. Nous remarquons aussi, et nous l'avons déjà noté au sujet des métacarpiens III, que dans les cas où la comparaison est possible, les os du Cheval de LunelViel se révèlent plus massifs. M.-F. Bonifay a d'ailleurs insisté sur la robustesse de cet Equidé.

\begin{tabular}{|c|c|c|c|c|c|}
\hline & \begin{tabular}{|c|}
$\begin{array}{c}\text { Longucur } \\
\text { externe en } \\
\text { mm }\end{array}$ \\
\end{tabular} & $\begin{array}{c}\text { Coefficients de } \\
\text { Kiesewalter } \\
(1889) \\
\end{array}$ & \begin{tabular}{|c|}
$\begin{array}{c}\text { Evaluation } \\
\text { minimum } \\
\text { en } \mathbf{~ c m ~}\end{array}$ \\
\end{tabular} & \begin{tabular}{|c|} 
Evaluation \\
maximum \\
en $\mathbf{~ c m}$ \\
\end{tabular} & $\begin{array}{c}\text { Evaluation } \\
\text { moyenne } \\
\text { en cm }\end{array}$ \\
\hline Humérus & 305,5 & $x 4,86(4,23-5,04)$ & 129,2 & 154,0 & 148,5 \\
\hline Radius & 349,5 & $\times 4,34(4,23-4,62)$ & 147,8 & 161,5 & 151,7 \\
\hline Métacarpien III & 238,7 & $\times 6,41,(5,99-6,90)$ & 743,0 & $.164,7$ & 153,0 \\
\hline Tibia & $\begin{array}{l}360.5 \\
361,0\end{array}$ & $x \quad 4,36(4,19.4,61)$ & $\begin{array}{l}151,0 \\
151,3\end{array}$ & & $\begin{array}{l}157.2 \\
157,4\end{array}$ \\
\hline Métatarsien III & 285,0 & $\times 5,33(4,89-5,73)$ & 139,4 & 163,3 & $\begin{array}{l}151,9 \\
151,9\end{array}$ \\
\hline & $\begin{array}{c}\begin{array}{c}\text { Longueur } \\
\text { basale en } \\
\text { mm }\end{array} \\
\end{array}$ & $\begin{array}{c}\text { Coefficients de } \\
\text { Nehring (in } \\
\text { Gromova.1949) }\end{array}$ & $\begin{array}{c}\begin{array}{c}\text { Fvaluation } \\
\text { minimum } \\
\text { en } \mathbf{~ c m ~}\end{array} \\
\end{array}$ & $\begin{array}{c}\begin{array}{c}\text { Evaluation } \\
\text { maximumn } \\
\text { en } \mathbf{~ c m ~}\end{array} \\
\end{array}$ & $\begin{array}{c}\begin{array}{c}\text { Evaluation } \\
\text { moyenne } \\
\text { en cm }\end{array} \\
\end{array}$ \\
\hline âne & 525,0 & $\times 3,00(2,75-3,25)$ & 144,4 & 170,6 & \\
\hline
\end{tabular}


Dans le matériel très abondant découvert à la Caune de l'Arago figurent peu de grands os longs. E. Crégut (1979) signale cependant la présence d'un métacarpien III de $263 \mathrm{~mm}$ et de deux métatarsiens III, I'un dépassant légèrement $290 \mathrm{~mm}$, l'autre ne mesurant que $285,9 \mathrm{~mm}$. L'auteur signale également (loc. cit. p.335) que, dans l'ensemble, les 22 talus "sont un peu plus hauts et larges" que ceux du Cheval de ChâtillonSaint-Jean et de La Micoque, tandis que les premières phalanges antérieures sont moins longues $(91,27 \mathrm{~mm}$ en moyenne) que celles de Mosbach $(93,63 \mathrm{~mm})$. L'Equidé de la Caune de l'Arago, d'abord nommé Equus mosbachensis tautavelensis par E. Crégut (1980) est rapproché du Cheval fossile de la presqu'île de Toungouze (Fédération de Russie) : Equus chosaricus Gromova, 1949 (Eisenmann et al, 1985).

II n'est pas d'ouvrage traitant des Equidés pléistocènes qui ne se réfère aux documents livrés par les alluvions rhénanes mindéliennes de Mosbach. II a été question plus haut de deux crânes relativement bien conservés mais nous connaissons aussi Equus mosbachensis par les diverses parties de son squelette appendiculaire représentées par de nombreux spécimens complets. Les mensurations pratiquées sur ces pièces par W. von. Reichenau (1915) et par G. Nobis (1971) indiquent un animal de haute taille, peut-être encore plus grand que ceux d'Achenheim et de la Caune de l'Arago et, a fortiori, que ceux de Châtillon-Saint-Jean, de LunelViel et de Camp-de-Peyre. La longueur moyenne de 28 os canons antérieurs, évaluée d'après les chiffres fournis par G. Nobis, atteint $258,17 \mathrm{~mm} \pm 3,06$ :

Elle dépasse donc la longueur du plus grand des 3èmes métacarpiens de Camp-de-Peyre. Ceux-ci seraient aussi relativement un peu moins massifs que leurs homologues du gisement allemand dont l'indice de robustesse oscille entre 15,3 et 18,2 et égale en moyenne $16,52 \pm 0,28$ $(n=26)$.

De ce qui précède il ressort que le Cheval de Camp-de-Peyre, aux formes relativement sveltes, était un animal de haute taille comparativement à ses congénères würmiens. Vraisemblablement moins grand que certains chevaux rissiens (cf. le Cheval des loess anciens d'Achenheim) et mindéliens (cf. le Cheval de Mosbach), il était sûrement plus élancé que l'Equidé de Lunel-Viel (interglaciaire Mindel/Riss).

A l'aide des coefficients déterminés par $L$. Kiesewalter pour les os longs et A. Nehring pour le crâne, nous avons évalué sa taille au sommet du garrot.

Très approximativement, le Cheval fossile de Sauveterre-la-Lémance devait mesurer en moyenne 1,50 à $1,55 \mathrm{~m}$ au garrot.
II-5) Caractères anatomiques remarquables de quelques os longs.

Si la morphologie de la tête osseuse, avec ses arcs dentaires, atteste l'appartenance de l'Equidé de Camp-de-Peyre au groupe des chevaux, il en va de même des autres parties du squelette. Pourtant nous avons relevé sur certaines d'entre elles quelques traits moins souvent rencontrés chez les formes plus récentes, les Chevaux würmiens en particulier. II s'agit, dans la grande majorité des cas, de dispositions considérées comme archaïques, ce qui n'est pas sans rappeler des observations effectuées sur le crâne et plusieurs portions de mâchoires.

II-5-1) Radius et ulna.

4 des 12 os dont la région distale est conservée ( $n^{\circ} 23,27,28$ et 34$)$ montrent dans le quart inférieur de la face palmaire du radius des vestiges, ténus certes mais à peu près continus, de la diaphyse de l'ulna.

A l'extrémité distale du spécimen $n^{\circ} 34$, l'incisure radio-ulnaire est si profonde que la face articulaire de l'ulna apparaît presque complètement séparée de celle du radius. Ce caractère se retrouve mais légèrement atténué sur l'épiphyse $n^{\circ} 33$ tandis que sur le spécimen $n^{\circ} 31$ la limite des deux os est "seulement» marquée par une rainure particulièrement accusée.

En général, chez le Cheval, à l'extrémité proximale du radius, le bord dorsal de la cavité glénoïde médiale est à peu près perpendiculaire dans son ensemble à l'axe longitudinal de l'os, moins souvent oblique de bas en haut dans le sens médio-latéral. Ce dernier caractère qui semble en revanche plus commun et plus net chez les Equidés du Pléistocène ancien s'observe sur 6 radius de Camp-de-Peyre (Fig.15c) tout en étant moins prononcé qu'il ne l'est habituellement chez Equus stenonis. En revanche il serait, selon toute vraisemblance, plus fréquent chez le Cheval fossile de Sauveterre-la-Lémance que chez la plupart des Chevaux du Pléistocène supérieur.

D'autre part, de façon quasi-systématique, sur le bord interne de la diaphyse, au dessous de la tubérosité bicipitale, la limite de la coulisse tendineuse du muscle brachial antérieur est tout à fait nette, plus nette qu'elle ne l'est le plus souvent chez les Chevaux würmiens et post-würmiens. En revanche cette disposition rappelle ce que l'on observe chez Equus stenonis (Prat, 1980, p.37 et Fig. $25 n^{\circ} 1 c$ et $\left.26 n^{\circ} 2\right)$.

\section{II-5-2) Les métacarpiens III \\ et métatarsiens III}

Les diagrammes relatifs à ces pièces ont été établis suivant le procédé déjà employé pour les crânes, c'est à dire, dans chaque cas, à partir de la différence entre deux logarithmes représentant l'un la moyenne des mesures se rapportant à l'Equidé de référence, ici Equus caballus arcelini du Magdalénien de Solutré, l'autre la moyenne 
d'une série de mesures se rapportant aux différentes formes considérées.

\section{II-5-2-1) Les métacarpiens III}

$1^{\circ}$ ) La facette de contact avec le trapézoïde n'attire l'attention que dans un seul cas $\left(n^{\circ} 22\right.$, Fig.15a). Sur les autres os canons elle est pratiquement inexistante ou de l'ordre du millimètre. M. F. Bonifay (1980) signale que cette facette est très inconstante sur les métacarpiens III de LunelViel.

$2^{\circ}$ ) Le graphique de la figure 16 ne fait pas apparaître de différence notable (sauf en ce qui concerne la taille) entre le Cheval de Mosbach et celui de Camp-de-Peyre. Cependant nous remarquons que chez ce dernier, la facette antérieure répondant à l'unciforme a un diamètre transversal maximum (mesure 8 ) relativement plus grand tandis que le diamètre transversal de la facette postérieure (mesure $8^{\prime}$ ) est relativement plus petit. D'après V. Eisenmann (1979, p.877 et p.880) Equus caballus mosbachensis serait plus évolué qu'Equus przewalskii car les deux facettes en contact avec l'unciforme sont proportionnellement plus vastes : il ne pourrait donc être son ancêtre. Or, chez le Cheval de Camp-de-Peyre, si les facettes articulaires du métacarpien III qui supportent l'unciforme sont également plus grandes que chez Equus przewalskii, comme chez ce dernier le diamètre transversal maximum de la facette postérieure est plus petit que celui de l'antérieure, disposition inverse de celle observée chez le Cheval de Mosbach.

$3^{\circ}$ ) L'indice de l'épaisseur proximale totale en fonction de la largeur proximale totale est élevé pour les 5 spécimens de Camp-de-Peyre : il varie de 66,7 à 73,3 avec une moyenne de 69,7 mais nous avons affaire à un petit effectif (Fig.16 et 17). Avec 81 métacarpiens III de Solutré provenant de fouilles anciennes, comptant donc des os d'Equus caballus gallicus et d'Equus caballus arcelini, on obtient les chiffres suivants : intervalle de variation :

$60,4-69,3$, moyenne $65,68 \pm 0,73$. Les valeurs correspondantes chez Equus stenonis (Prat, 1980) sont les suivantes : Equus stenonis vireti de Saint-Vallier : 63,2-71,3 (moyenne de 67,87 pour un effectif de 20), Equus stenonis de Senèze: 64,8-72,3 (moyenne de $68,20 \pm 1,42$ pour un effectif de 12).

II-5-2-2) L'extrémité distale des métacarpiens III et métatarsiens III.

Les figures $16,17,18,19$ et les tableaux 17 et 24 montrent que les tubercules sus-articulaires latéraux de l'extrémité distale des os canons, antérieurs et postérieurs, sont plus développés chez les Chevaux ayant vécu en Europe occidentale antérieurement au Riss III (ceux de Campde-Peyre ne font pas exception) que chez les Chevaux plus récents. En ce qui concerne les métatarsiens III il est fréquent que le rapport centésimal du diamètre transversal sus-articulaire au diamètre transversal articulaire atteigne presque ou égale 100 et parfois même dépasse cette valeur (cf. les métatarsiens III de Mosbach, Montmaurin, Lunel-Viel, La Caune de l'Arago, Steinheim, Châtillon-Saint Jean, Camp-dePeyre).

On sait que chez Equus stenonis la largeur de l'os mesurée au niveau des tubercules latéraux surpasse généralement le diamètre bicondylien non seulement sur les métatarsiens III mais aussi sur les métacarpiens III. Les os canons des chevaux du Pléistocène moyen auraient donc conservé plus nettement que ceux des chevaux du Pléistocène supérieur, le souvenir d'une disposition encore très accusée chez la plupart des Equus du Pléistocène ancien. Si cette observation était confirmée elle présenterait un intérêt certain du point de vue chronologique.

\section{III) CONCLUSIONS}

La morphologie des vestiges d'Equidés provenant du remplissage de la faille ouverte en 1976 à Camp-de-Peyre inspire d'emblée une première remarque : ces documents se rapportent à un caballin au sens large. Certains caractères dentaires, comme par exemple l'allongement du protocône des arrières-molaires et prémolaires supérieures, le dédoublement à peu près constant du parastyle et du métastyle de ces dernières, ainsi que la forme de la double-boucle des mâchelières inférieures, imposent en effet cette diagnose. Presque aussi évidente est la haute stature de l'animal, ce que ne tardent d'ailleurs pas à confirmer les mesures pratiquées sur les parties du squelette les mieux conservées. Elles indiquent une taille d'au moins $1,50 \mathrm{~m}$ au garrot ce qui, pour un Cheval fossile, est le signe d'une certaine ancienneté et, dans le cas qui nous occupe, s'accorde bien avec l'ensemble des données paléontologiques qui annoncent un âge Pléistocène moyen.

Un examen plus attentif des documents révèle un certain nombre de caractères non mesurables dont nous ne savons trop s'ils doivent ou non jouer un rôle dans la définition de notre Equidé : les uns n'apparaissent pas de façon constante, les autres affectent l'unique tête osseuse découverte.

Parmi les premiers il faut signaler :

- l'apparition des dents de loup ( $\left.\mathrm{D}^{1}-\mathrm{P}^{1}\right)$ supé- 
rieures et inférieures, qui pourrait être plus fréquente que chez les caballins plus récents(4),

- la forme de la 3ème molaire inférieure à bord distal parfois fortement concave en vue linguale ou vestibulaire,

- la régression non accomplie de l'ulna sur quelques rares spécimens d'os radio-ulnaires,

- certaines particularités du radius.

En ce qui concerne la tête osseuse nous avons surtout remarqué :

- la probable ensellure fronto-nasale du profil supérieur et l'existence d'une gouttière le long de la suture des os nasaux,

- la forme de la branche montante mandibulaire dont le bord caudal, au-dessous du condyle, fuit vers l'avant ce qui n'est pas fréquemment le cas chez les Chevaux du Pléistocène supérieur.

Parmi les caractères considérés comme archaïques il en est deux difficilement contestables en raison de leur quasi-constance. II s'agit de l'absence ou de l'insignifiance de la facette articulaire qui, chez la majorité des chevaux würmiens et actuels, répond au trapézoïde et de la saillie des tubercules latéraux sus-articulaires de l'extrémité distale des os canons qui se révèle moins accentuée que chez les Equus du Pléistocène ancien mais plus accusée que chez les Chevaux contemporains de la dernière glaciation.

Nous fondant sur les données de la littérature et notre propre expérience, nous sommes conduits à penser que le développement de ces reliefs, tel que nous le constatons sur les métacarpiens et métatarsiens III de Camp-de-Peyre, est selon toute probabilité l'une des caractéristiques des grands chevaux anté-würmiens, hormis peut-être ceux ayant vécu soit au cours du dernier interglaciaire, soit lors des derniers épisodes rissiens.

Nous devons aussi insister sur la présence, déjà signalée, d'un autre caractère qui se manifeste de façon à peu près systématique chez les grands chevaux que nous venons d'évoquer. $\mathrm{Ce}$ caractère est lié à l'évolution de l'indice protoconique dans la série des dents jugales supérieures (Eisenmann, 1980 et Guadelli et Prat, 1983). De $\mathrm{P}^{2}$ à $\mathrm{P}^{4}$ cet indice progresse, puis il reste stable ou diminue légèrement sur $M^{1}$ pour augmenter à nouveau de $\mathrm{M}^{1}$ à $\mathrm{M}^{3}$. Rien cependant, dans ce cas, ne nous autorise à dire pour l'instant qu'il s'agit d'une disposition archaïque. A partir du Riss III l'indice protoconique moyen des chevaux préhistoriques croît de $\mathrm{P}^{2}$ à $\mathrm{M}^{2}$ puis chute sur $\mathrm{M}^{3}$.
V. Eisenmann, E. Crégut-Bonnoure et A.-M. Moigne (1985) rappellent que chez les chevaux, comme chez d'autres mammifères, le raccourcissement du museau, qui tend à limiter les pertes de chaleur, peut constituer une adaptation à des conditions climatiques froides. Ces auteurs citent en exemple, entre autres, le Cheval de la Caune de l'Arago et Equus chosaricus de Toungouze dont il a déjà été question. L'Equidé de Camp-dePeyre, qui appartient à un ensemble faunique à Renne dominant assez semblable à celui de la Caune de l'Arago, en serait une autre illustration.

Quoi qu'il en soit le Cheval de Camp-dePeyre, soumis à des conditions steppiques froides, semble différer sensiblement de celui de Lunel-Viel, moins grand, aux formes lourdes, au museau modérément large et étroit qui, selon M.F. Bonifay (1980), évoluait dans un milieu tempéré et humide.

Les études paléontologiques traitant du Pléistocène moyen se heurtent à un certain nombre de difficultés parmi lesquelles la détermination de l'âge des documents n'est certainement pas la moindre. La sensibilité encore insuffisante des méthodes physico-chimiques de datation n'apporte généralement pas, pour cette période, de solution satisfaisante au problème. Aussi arrive-t-il que telle faune datée par certains du Mindel soit considérée comme rissienne par d'autres. Entre les deux évaluations, dans l'hypothèse la plus optimiste, il n'y a seulement, il est vrai, que la durée d'un interglaciaire! L'imprécision de la position chronologique de Camp-de-Peyre n'en constitue qu'un nouvel exemple.

Une deuxième difficulté tient à la rareté des ensembles fossilifères se rapportant à cette longue période et au fait que certaines pièces ne sont qu'exceptionnellement conservées. C'est notamment le cas des crânes de chevaux, rappelons-le, puisque 2 spécimens ont été découverts en France l'un à Lunel-Viel et l'autre à Camp-dePeyre. La portée des comparaisons dès lors se trouve fort limitée.

La réserve dont nous faisons preuve dans nos conclusions nous est dictée non seulement par les carences de notre documentation mais aussi par ce que l'on sait des faunes qui se sont succédé aux cours des cent cinquante derniers millénaires en réponse aux transformations parfois profondes de l'environnement. II paraît peu probable que les temps préhistoriques plus anciens aient été moins fertiles en changements dans ce domaine.

4. Nous remarquerons que sur les 3 séries dentaires supérieures représentées par W. von Reichenau (1915, pl. VIII n³, 4a, 4b) 2 comportent une D1(P1) et qu'un des 2 crânes figurés par cet auteur possédait une dent de loup, au moins du côté droit (loc. cit., planche $\left.X I, n^{\circ} 5\right)$. 
Enfin nos observations nous amènent à reposer la question de la position taxinomique du Cheval fossile lot-et-garonnais et, plus généralement, des Chevaux dits de Mosbach. Si, à l'évidence, il ne s'agit ni de Zébrins, ni d'Asiniens, peut-on les classer parmi les Caballins (stricto sensu) ou les distinguer d'Equus caballus suivant en cela V. Eisenmann (1980) et M.-F. Bonifay (1980) ? Le problème n'est pas simple à résoudre car, si la morphologie générale des dents et des os indique que nous avons affaire dans chaque cas à un Cheval (lato sensu), de nombreux caractères, souvent archaïques, distinguent les grands Equidés du Pléistocène moyen des Chevaux plus récents. Nous avons vu précédemment qu'il existe au sein du groupe "Mosbach" un polymorphisme certain dont l'interprétation n'est pas aisée. En conséquence, nous pensons comme les auteurs précités, qu'il est préférable de considérer que ces Chevaux appartiennent à la même espèce, Equus mosbachensis, et de donner à chaque morphotype un rang sous-spécifique. A l'appui de cette opinion notons qu'il existe plusieurs espèces parmi les Zébrins ainsi que l'ont souligné V. Eisenmann, E. Crégut-Bonoure et A.M. Moigne (1985, p.157).

L'étude du Cheval de Camp-de-Peyre nous a donc amenés à préciser la définition de l'espèce Equus mosbachensis. A la lumière de nos observations la situation taxinomique de certains Equidés comme ceux de Châtillon-Saint Jean et peut-être de Steinheim mériterait d'être révisé.

En tout cas nous croyons devoir donner au Cheval fossile de Camp-de-Peyre une place bien déterminée dans la nomenclature des Equidés pléistocènes.

Equus mosbachensis campdepeyri nov.ssp.

Holotype : Crâne ${ }^{\circ} 234$ couche 2

Paratype : Mâchoires complètes n ${ }^{\circ} 127$ c.3 et 252 c. 3

Localité-type : Camp-de-Peyre à Sauveterre-laLémance

Niveau-type : couche 2 et 3

Diagnose :

$1^{\circ}$ ) Cheval d'environ $1,54 \mathrm{~m}$ de hauteur au garrot,

$2^{\circ}$ ) Crâne. Caractères présents sur l'holotype:

- Museau court et face longue,

- Profil supérieur à ensellure fronto-nasale,

- Gouttière internasale présente mais peu accusée.

$\left.3^{\circ}\right)$ Mandibule :

- Bord distal de la branche montante oblique vers l'avant, au dessous du condyle.

$\left.4^{\circ}\right)$ Denture :

- Morphologie typiquement caballine mais à protocône peu allongé sur $M^{1}$ et relativement développé sur $\mathrm{M}^{3}$,

- Bord distal de la $M^{3}$ inférieure plus ou moins concave en vue linguale et vestibulaire. $\left.5^{\circ}\right)$ Squelette appendiculaire :

- Région proximale du radius :

Obliquité du bord antérieur de la face articulaire médiale fréquente,

Limite de la coulisse tendineuse du muscle brachial antérieur souvent bien marquée.

- Os canons

Tubercules latéraux sus-articulaires de l'extrémité distale plus proéminents que chez les chevaux plus récents.

Métacarpiens III : facette articulaire répondant au trapézoïde absente ou de dimensions très réduites.

Métatarsiens III : facette articulaire répondant au petit cunéiforme absente ou de dimensions très réduites. 


\section{BIBLIOGRAPHIE}

BARONE R. (1966) - Anatomie comparée des Mammifères domestiques. t.1 : Ostéologie. Laboratoire d'anatomie. Ecole Vétérinaire, Lyon, 811p., très nombreuses ill.

BONIFAY M.-F. (1968) - Carnivores quaternaires du Sud-Est de la France. Thèse de Doctorat ès-Sciences naturelles de la Faculté des Sci. de Paris, $n^{\circ}$ A.0.21-02, 3 tomes, 366p., 62 pl., 99 tab.

BONIFAY M.-F. (1971) - Carnivores quaternaires du Sud-Est de la France. Mémoires du Muséum National d'histoire naturelle, Paris. Nile série, (C), Sciences de la Terre, t.XXI, fasc. 2 et dernier. 377p., 76 fig., 109 tab., XXVII pl.

BONIFAY M.-F. (1980) - Le Cheval du Pléistocène moyen des grottes de Lunel-Viel (Hérault) Equus mosbachensis palustris, nov. ssp. Gallia-Préhistoire, Paris, t.23, $\mathrm{n}^{\circ} 2$, pp.233281, 24 fig., 33 tab.

BORDES F. (1960) - Compte rendu de l'ouvrage de $P$. Wernert «stratigraphie paléontologique et préhistorique des sédiments quaternaires d'Alsace Achenheim". L'Anthropologie, Paris. t.64, n¹-2, pp.77-85.

CAILLAT P. (1994) - La faune des milieux clos dans l'Antiquité gallo-romaine. Thèse de Doctorat d'Université $n^{\circ} 8217856 C$, Univ. Bordeaux III, Michel Montaigne, 2 tomes, t.1 : 582p., t.2 : 171p., nombreux ill. et tab.

CHAUVIRÉ C. (1962) - Les gisements fossilifères de Châtillon-Saint-Jean (Drôme). Thèse de Doctorat de 3ème cycle, Faculté des Sciences de Lyon, 216p., 19 fig., 2 pl.

CRÉGUT E. (1979) - La faune de mammifères du Pléistocène moyen de la Caune de l'Arago à Tautavel, Pyrénées-Orientales. Thèse de 3ème cycle de l'Univ. de Provence, Marseille, 3 tomes, 381p., 48 pl., 193 tab.,Trav. Lab. Paléont. hum. Préhist., Marseille, (3), fasc.1-3.

CRÉGUT E. (1980) - Equus mosbachensis tautavelensis nov. subsp., un nouvel Equidae (Mammalia, Perissodactyla) du gisement pléistocène moyen anté-rissien de la Caune de l'Arago (Tautavel, Pyrénées-Orientales, France). Géobios, Lyon, $\mathrm{n}^{\circ} 13,(1), \mathrm{pp} .121-$ 127, 2 tab., 2 pl.

DELPECH F., LE TENSORER J.-M., PRAT F., PINÉDA R. (1978) - Un nouveau gisement du Pléistocène moyen : Camp de Peyre à Sauveterre-la-Lémance (Lot-et-Garonne). Comptes Rendus de l'Académie des Sciences, Paris, t.286, (D), pp.1101-1103
DONARD E. (1982) - Recherches sur les Léporinés quaternaires (Pléistocène moyen et supérieur, Holocène). Thèse de 3ème cycle de I'Univ. Bordeaux I, n¹764, 2 tomes, 161p., 46 pl., 89 tab.

EISENMANN V. (1978) - Les chevaux (Equus sensu lato) fossiles et actuels: étude crâniologique et odontologique. Thèse de Doctorat d'état, ès Sciences Naturelles, Université Pierre et Marie Curie, Paris, 444p., 121 fig., 106 tab., 28 pl.

EISENMANN V. (1979) - Les métapodes d'Equus sensu lato (Mammalia, Perissodactyla). Géobios, Lyon, n¹2, fasc.6, pp.863-886, 19 fig., 11 tab.

EISENMANN V. (1980) - Les chevaux (Equus sensu lato) fossiles et actuels: étude craniologique et odontologique. Cahiers de Paléontologie, Ed. du CNRS, Paris, 186p., 67 fig., 72 tab., 22 pl.

EISENMANN V., CRÉGUT-BONOURE E., MOIGNE A.-M. (1985) - Equus mosbachensis et les grands Chevaux de la Caune de l'Arago et de Lunel-Viel : crâniologie comparée. Bulletin du Muséum National d'histoire naturel, Paris, (4), $n^{\circ} 7,1985$, section C, $n^{\circ} 2$, pp.157173, 5 fig., 2 tab.

GOUBAUX A., BARRIER G. (1884) - De l'extérieur du Cheval. Asselin et Cie éditeurs, Paris. (1ère édition), 1067p., 293 fig., 33 pl.

GOUBAUX A., BARRIER G. (1890) - L'extérieur du Cheval. Asselin et Houzeau éd., Paris. (2ème édition), 996p., 346 fig., 34 pl.

GROMOVA V. (1949) - Histoire des chevaux (genre Equus) de l'ancien monde (1ère partie). Revue et description des formes. Travaux Institut de Paléontologie de l'Académie des Sciences URSS, $n^{\circ} 17, \quad n^{\circ} 1$. Traduction Pietresson de Saint-Aubin. Annales du Centre d'Etudes et de documentation paléontologiques, $1955, \mathrm{n}^{\circ} 13$.

GUADELLI J.-L., PRAT F. (1983) - Les vestiges d'Equidés de l'aven de Vergranne (Doubs). Annales Scientifiques de l'Université de Besançon. Géologie, 4ème série, fasc.5, pp.83-92, 2 fig., 6 tab.

GUADELLI J.-L. (1986) - Révision de la sousespèce Equus caballus gallicus. Contribution du Cheval à la connaissance des paléoenvironnements. 11ème RST, 25-27 Mars 1986, Clermont-Ferrand.

GUADELLI J.-L. (1987) - Contribution à l'étude des zoocénoses préhistoriques en Aquitaine (Würm ancien et interstade würmien). Thèse 
de Doctorat de l'Université Bordeaux I, $\mathrm{n}^{\circ} 148$, 3 tomes, 568p., 163 fig., 424 tab.

GUADELLI J.-L. (1989) - Les Equidés. in La Baume de Gigny (Jura). Sous la dir. M. Campy, J. Chaline, M. Vuillemey. XXVIlème suppl. à Gallia-Préhistoire, Paris, pp.89-95, fig.37 à 39, tab.XIX à XXI.

GUADELLI J.-L. (1990) - Quelques données sur la faune de Coupe-Gorge à Montmaurin (Haute-Garonne, France). Paléo, Ed. SAMRA, Les Eyzies de Tayac. n², pp.107-126, 2 fig., 56 tab.

GUADELLI J.-L. (1991) - Les Chevaux de Solutré (Saône-et-Loire, France). Actes des Symposium de la 11ème RST, ClermontFerrand, 1986 "Datation et Caractérisation des Milieux Pléistocènes". Cahiers du Quaternaire. Ed. CNRS, Paris. n¹6, pp.261336, 9 fig., 62 tab., 1 ann.

KIESEWALTER L. (1889) - Skelettmessungen am Pferde. Dim. Leipzig.

LE TENSORER J.-M. (1973) - Le gisement du Martinet à Sauveterre-la-Lémance (Lot-etGaronne). Etude géologique et géochimique. Bulletin AFEQ, Paris, pp.215-237, 16 fig., 2 tab.

LE TENSORER J.-M. (1981) - Le Paléolithique de l'Agenais. Cahier du Quaternaire, Ed. du CNRS, Paris, $n^{\circ} 3,526$ p., 55 tab., 212 fig.

MARQUET J.-Cl. (1993) - Paléoenvironnement et chronologie des sites du domaine atlantique français d'âge pléistocène moyen et supérieur d'après l'étude des rongeurs. Tours, 1993, 345p., 120 fig., 233 tab.

MOURER-CHAUVIRÉ C. (1972) - Etude de nouveaux restes de vertébrés provenant de la carrière Fournier à Châtillon-Saint-Jean (Drôme). III Artiodactyles, Chevaux, Oiseaux. Bulletin de l'Association Française d'Etude du Quaternaire, Paris. t.33, nº, pp.271-305, 3 pl.

MOURER-CHAUVIRÉ C. (1980) - Le gisement pléistocène supérieur de la grotte de Jaurens à Nespouls, Corrèze ; les Equidés. Nouvelles Archives du Muséum d'Histoire naturelle de Lyon, Lyon, fasc.3, pp.17-60, 6 figs., 28 tab., 5 pl.

NOBIS G. (1971) - Vom Wildpferd zum Hauspferd. Böhlau Verlag Köln, Wien. 96p., Tafeln 1-6, Diagramme 1-58, Ma tabellen 1CXXVI.

PETIT M. (1939) - Anatomie des molaires des Equidés, Cheval et Ane. Thèse Faculté des Sciences de Paris, 1939 et Lio et Fils, Toulouse, 328p., 206 fig., 110 tabl.
POMMEROL F. (1879) - Le Mouflon Quaternaire. A.F.A.S., Montpellier, pp.600-609, fig.67-71, pl.III

POMMEROL F. (1881) - Recherche sur le Mouflon quaternaire (Ovis antiqua). A.F.A.S., Alger. pp.525-530, fig.68-71.

PRAT F. (1968) - Recherches sur les Equidés pléistocènes en France. Thèse de Doctorat ès Sciences Naturelles, $n^{\circ} 226$, Faculté des Sciences de Bordeaux, 4 volumes, 696p., 149 fig., 126 tab.

PRAT F. (1977) - L'Equidé du gisement Acheuléen de Torralba (Soria, Espagne). Equus caballus torralbae. nov. subsp. Recherches françaises sur le Quaternaire, INQUA, 1977, supp. Bulletin de l'Association Française d'Etude du Quaternaire, Paris. t.50, $\mathrm{n}^{\circ} 1$, pp.33-46, 6 tab., 3 pl.

PRAT F. (1980) - Les Equidés villafranchiens en France. Cahiers du Quaternaire, Ed. CNRS, Paris, n², 290p., 55 fig., 46 tab.

PRAT F., THIBAULT CI. (1976) - Le gisement de Nauterie à La Romieu (Gers). Fouilles de 1967 à 1973. Nauterie I. Mémoire du Muséum National d'Histoire naturelle, Paris, nouvelle série, (C), t.XXXV, 96 p., 35 fig., 7 pl. h. t.

REICHENAU W. VON (1915) - Beitrage zur näheren Kenntnis fossiler Pferde aus deutschem Pleistozän insbesondere über die Entwicklung die Abkaustadien des Gebisses vom Hochterrassenpferd (Equus mosbachensis v. R.). Abhandlungen der grossherzolich Hessischen Geologischen Landesanstalt zu Darmstadt, band VII, Heft 1, pp.1-155, nbreux tab., 14 pl.

RUDEL A. (1953) - La faune quaternaire des terrasses de l'Allier à Pont-du-Château. Revue des Sciences naturelles d'Auvergne, pp.43-47

SIMPSON G.G. (1941) - Large pleistocene felines of North America. Am. Mus. Novitates, NewYork, n¹136, pp.1-27, 11 fig.

WERNERT P. (1957) - Stratigraphie paléontologique et préhistorique des sédiments quaternaires d'Alsace : Achenheim. Mémoire du Service de la carte Géologique d'Alsace et de Lorraine, Strasbourg, $n^{\circ} 14$, pp.1-254, 111 fig., 24 pl., 1 carte, 6 coupes. 
Dans les tableaux ci-après figurent dans l'ordre :

le nombre de données

l' intervalle de variation

La moyenne et son intervalle de confiance si le nombre de données est supérieur à 5

Lorsque le nombre de données est égal à 3 la moyenne, entre parenthèses, n'est donnée qu'à titre indicatif.

Signification des abréviations utilisées dans les tableaux :

DAP : Diamètre antéro-postérieur

DDP : Diamètre dorso-plantaire ou dorso-palmaire

DMDalv : Diamètre mésio-distal au niveau alvéolaire

DMDo : Diamètre mésio-distal au niveau occlusal

DMDp : Diamètre mésio-distal au niveau du point «p»

DMDpF : Diamètre mésio-distal du post-flexide

DMDpr.o : Diamètre mésio-distal du protocône au niveau occlusal

DMDpr.p : Diamètre mésio-distal du protocône au niveau du point «p»

DT : Diamètre transversal

DVLo : Diamètre vestibulo-lingual au niveau occlusal

DVLp : Diamètre vestibulo-lingual au niveau du point «p»

IF : Indice flexidique

IPo : Indice protoconique au niveau occlusal

IPp : Indice protoconique au niveau du point «p»

IR : Indice de robustesse

prox : proximal

tub : tubérosité

\begin{tabular}{|l|c|}
\hline 1:Longueur basilaire & 525,0 \\
\hline 2:Longueur palatine & 285,0 \\
\hline 5:Longueur du museau & 129,0 \\
\hline 2-5:Longueur du palais & 156,0 \\
\hline 6:Longueur du diastème & 110,0 \\
\hline 7:Diamètre mésio-distal P2-P4 & 103,3 \\
\hline 8:Diamètre mésio-distal P2-M3 & 187,0 \\
\hline 13:Largeur frontale & 219,5 \\
\hline 17:Largeur du prémaxillaire & 78,0 \\
\hline 17b:Largeur minimum du museau & - \\
\hline 21:Diamètre antéro-postérieur de l'orbite & 64,0 \\
\hline 23:Longueur de la ligne oculaire antérieure & 395,0 \\
\hline 24:Longueur de la ligne oculaire postérieure & 200,0 \\
\hline
\end{tabular}

Tableau 1 : Camp-de-Peyre Dimensions du crâne $n^{\circ} 234$ 


\begin{tabular}{|l|c|c|c|}
\hline & $\mathrm{I}^{\mathrm{I}}$ & $\mathrm{I}^{2}$ & $\mathrm{I}^{3}$ \\
\hline DMDo & 4 & 4 & 4 \\
& $19,0-23,0$ & $20,7-22,8$ & $21,0-22,5$ \\
& 21,1 & 21,8 & 21,8 \\
\hline \multirow{2}{*}{ DVLo } & 3 & 4 & 4 \\
& $\begin{array}{c}12,0-13,2 \\
(12,7)\end{array}$ & $\begin{array}{c}10,8-12,5 \\
11,8\end{array}$ & $\begin{array}{c}11,0-11,8 \\
11,5\end{array}$ \\
\hline
\end{tabular}

Tableau 2: Camp-de-Peyre - Dimensions des incisives et canines supérieures

\begin{tabular}{|l|c|c|c|c|}
\hline & $\mathrm{P}^{2}$ & $\mathrm{P}^{3}$ & $\mathrm{P}^{4}$ & $\mathrm{P}^{3-4}$ \\
\hline \multirow{2}{*}{ DMDo } & $\mathbf{8}$ & 6 & 5 & 11 \\
& $39,0-42,8$ & $32,0-36,0$ & $31,4-35,0$ & $31,4-36,0$ \\
& $41,0 \pm 0,9$ & $34,3 \pm 1,5$ & 33,3 & $33,8 \pm 0,9$ \\
\hline \multirow{2}{*}{ DVLo } & $\mathbf{8}$ & 6 & 5 & 11 \\
& $25,1-30,0$ & $30,0-33,5$ & $29,0-33,4$ & $29,0-33,5$ \\
& $28,3 \pm 1,0$ & $31,9 \pm 1,2$ & 31,2 & $31,6 \pm 1,0$ \\
\hline \multirow{2}{*}{ DMDpr.o } & 8 & 6 & 5 & 11 \\
& $8,9-11,7$ & $14,4-17,0$ & $15,0-17,5$ & $14,4-17,5$ \\
& $10,2 \pm 0,8$ & $15,4 \pm 0,9$ & 16,0 & $15,7 \pm 0,6$ \\
\hline \multirow{2}{*}{ IPo } & 8 & 6 & 5 & 11 \\
& $21,0-27,9$ & $42,0-47,2$ & $46,1-50,0$ & $42,0-50,0$ \\
& $24,8 \pm 1,8$ & $45,0 \pm 1,5$ & 47,9 & $46,3 \pm 1,2$ \\
\hline \multirow{2}{*}{ DMDp } & 5 & 3 & 3 & 6 \\
& $38,0-42,7$ & $34,0-35,8$ & $33,0-34,6$ & $33,0-35,8$ \\
& 40,3 & $(35,1)$ & $(34,0)$ & $34,8 \pm 0,8$ \\
\hline \multirow{2}{*}{ IVVIp } & 5 & 3 & 2 & 5 \\
& $27,0-30,0$ & $31,7-33,7$ & $34,0-33,4$ & $31,7-34,0$ \\
& 28,5 & $(33,0)$ & & 33,3 \\
\hline \multirow{2}{*}{ DMDpr.p } & 5 & 3 & 3 & 6 \\
& $10,3-12,0$ & $14,2-15,5-$ & $15,0-16,5$ & $14,2-16.5$ \\
& 11,2 & 16,0 & $(16,0)$ & $15,6 \pm 0,7$ \\
\hline \multirow{2}{*}{ IPp } & 5 & 3 & 3 & 6 \\
& $26,3-28,9$ & $41,8-44,7$ & $45,5-47,8$ & $41,8-47,8$ \\
& 27,7 & $(43,4)$ & $(47,0)$ & $45,2 \pm 1,9$ \\
\hline
\end{tabular}

Tableau 3 : Camp-de-Peyre - Dimensions des prémolaires supérieures

\begin{tabular}{|l|c|c|c|c|}
\hline & $\mathrm{M}^{\mathrm{I}}$ & $\mathrm{M}^{2}$ & $\mathrm{M}^{1-2}$ & $\mathrm{M}^{3}$ \\
\hline \multirow{2}{*}{ IMIo } & 9 & 6 & 15 & 6 \\
& $28,0-31,5$ & $28,2-31,5$ & $28,0-31,5$ & $25,5-32,0$ \\
& $29,5 \pm 0,8$ & $30,1 \pm 1,0$ & $29,8 \pm 0,6$ & $29,1 \pm 2,1$ \\
\hline \multirow{2}{*}{ DVLo } & 9 & 5 & 14 & 6 \\
& $28,5-31,2$ & $26,5-29,5$ & $26,5-31,2$ & $20,2-26,5$ \\
& $29,8 \pm 0,6$ & 28,3 & $29,3 \pm 0,6$ & $23,9 \pm 2,1$ \\
\hline \multirow{2}{*}{ DMDpr.o } & 9 & 6 & 15 & 6 \\
& $13,0-15,1$ & $13,5-16,7$ & $13,0-16,7$ & $14,4-16,5$ \\
& $14,4 \pm 0,5$ & $15,3 \pm 1,1$ & $14,7 \pm 0,5$ & $15,5 \pm 0,7$ \\
\hline \multirow{2}{*}{ IPo } & 9 & 6 & 15 & 6 \\
& $45,1-53,9$ & $47,9-54,8$ & $45,1-54,8$ & $50,0-58,8$ \\
& $48,9 \pm 1,9$ & $50,6 \pm 2,5$ & $49,5 \pm 1,4$ & $53,3 \pm 3,0$ \\
\hline \multirow{2}{*}{ DMIDp } & 9 & 3 & 12 & 3 \\
& $28,0-30,5$ & $29,9-30,2$ & $28,0-30,5$ & $32,8-33,0$ \\
& $28,7 \pm 0,6$ & $(30,0)$ & $29,0 \pm 0,5$ & $(32,9)$ \\
\hline \multirow{2}{*}{ DVLp } & 8 & 3 & 11 & 3 \\
& $29,2-31,0$ & $28,0-31,0$ & $28,0-31,0$ & $25,0-26,5$ \\
& $30,1 \pm 0,4$ & $(29,7)$ & $30,0 \pm 0,5$ & $(26,0)$ \\
\hline \multirow{2}{*}{ DMDpr.p } & $13,2-15,1$ & $14,9-15,7$ & $13,2-15,7$ & $16,0-17,7$ \\
& $14,0 \pm 0,4$ & $(15,4)$ & $14,4 \pm 0,4$ & $(16,9)$ \\
\hline \multirow{2}{*}{ IPp } & 7 & 3 & 10 & 3 \\
& $44,0-53,9$ & $49,3-52,5$ & $44,0-53,9$ & $48,8-54,0$ \\
& $48,7 \pm 2,6$ & $(51,2)$ & $49,4 \pm 1,9$ & $(51,5)$ \\
\hline
\end{tabular}

Tableau 4 : (amp-de-Peyre - Dimensions des molaires supérieures

\begin{tabular}{|c|c|c|c|c|c|c|c|c|}
\hline & $\mathrm{P}^{2}$ & $\mathrm{P}^{3}$ & $\mathrm{P}^{4}$ & $\mathrm{P}^{3-4}$ & $\mathrm{M}^{\mathrm{I}}$ & $\mathrm{M}^{2}$ & $\mathrm{M}^{1-2}$ & $\mathrm{M}^{3}$ \\
\hline Camp-de-Peyre (1) & $\begin{array}{c}8 \\
24,8\end{array}$ & $\begin{array}{c}6 \\
45,0\end{array}$ & $\begin{array}{c}5 \\
47,9\end{array}$ & 11 & $\begin{array}{c}9 \\
48,9\end{array}$ & $\begin{array}{c}6 \\
50,6\end{array}$ & $\begin{array}{c}15 \\
49,5\end{array}$ & $\begin{array}{c}6 \\
53,3\end{array}$ \\
\hline $\begin{array}{l}\text { Moskach (2) } \\
\text { (Eisenmann, 1980) }\end{array}$ & $\begin{array}{c}11 \\
24,4\end{array}$ & $\begin{array}{c}14 \\
45,3 \\
\end{array}$ & $\begin{array}{c}15 \\
48,4 \\
\end{array}$ & $\begin{array}{c}29 \\
46,9 \\
\end{array}$ & $\begin{array}{c}14 \\
48,5\end{array}$ & $\begin{array}{c}13 \\
53,4 \\
\end{array}$ & $\begin{array}{c}27 \\
50,8 \\
\end{array}$ & $\begin{array}{c}7 \\
50,8 \\
\end{array}$ \\
\hline $\begin{array}{l}\text { Montoussé (3) } \\
\text { (Prat, 1968) }\end{array}$ & & & & 46,4 & & & 48,0 & 50,3 \\
\hline $\begin{array}{l}\text { La Micoque (4) } \\
\text { (Prat, 1968) }\end{array}$ & & & & 44,3 & & & 49,6 & 51,5 \\
\hline $\begin{array}{l}\text { Tilloux (5) } \\
\text { (Prat, 1968) }\end{array}$ & $\begin{array}{c}1 \\
27,0\end{array}$ & $\begin{array}{c}2 \\
39,3\end{array}$ & $\begin{array}{c}2 \\
47,7\end{array}$ & $\begin{array}{c}4 \\
43,5\end{array}$ & $\begin{array}{c}2 \\
46,5\end{array}$ & $\begin{array}{c}2 \\
44,2\end{array}$ & $\begin{array}{c}4 \\
45,4\end{array}$ & $\begin{array}{c}2 \\
44,6\end{array}$ \\
\hline $\begin{array}{l}\text { Vergramue (6) } \\
\text { (Guadelli, Prat, 1983) }\end{array}$ & & $\begin{array}{c}2 \\
46,7\end{array}$ & $\begin{array}{c}2 \\
50,2\end{array}$ & 48,5 & $\begin{array}{c}2 \\
49,7\end{array}$ & $\begin{array}{c}2 \\
54,2\end{array}$ & 52,0 & $\begin{array}{c}1 \\
53,8\end{array}$ \\
\hline $\begin{array}{l}\text { Lunel-Viel (7) } \\
\text { (Bonifay, 1980) }\end{array}$ & $\begin{array}{c}7 \\
23,7\end{array}$ & $\begin{array}{c}2 \\
40,5\end{array}$ & $\begin{array}{c}2 \\
47,0\end{array}$ & $\begin{array}{c}7 \\
43,3\end{array}$ & $\begin{array}{c}3 \\
47,3\end{array}$ & $\begin{array}{c}2 \\
50,0\end{array}$ & $\begin{array}{c}4 \\
48,0\end{array}$ & $\begin{array}{c}6 \\
49,0\end{array}$ \\
\hline $\begin{array}{l}\text { La Suard (A) (pt.p) } \\
\text { (Prat, 1968) }\end{array}$ & & $\begin{array}{c}13 \\
49,1\end{array}$ & $\begin{array}{c}7 \\
52,4\end{array}$ & $\begin{array}{c}26 \\
49,9\end{array}$ & $\begin{array}{c}11 \\
55,1\end{array}$ & $\begin{array}{c}8 \\
57,5\end{array}$ & $\begin{array}{c}28 \\
55,7\end{array}$ & $\begin{array}{c}12 \\
53,0\end{array}$ \\
\hline $\begin{array}{l}\text { Combe-Grenal (R) } \\
\text { (Guadelli, 1987) }\end{array}$ & $\begin{array}{c}10 \\
25,8\end{array}$ & $\begin{array}{c}39 \\
43,9\end{array}$ & $\begin{array}{c}32 \\
48,0\end{array}$ & $\begin{array}{c}71 \\
45,7\end{array}$ & $\begin{array}{c}41 \\
51,5\end{array}$ & $\begin{array}{c}23 \\
53,4\end{array}$ & $\begin{array}{c}70 \\
52,7\end{array}$ & $\begin{array}{c}46 \\
52,1\end{array}$ \\
\hline $\begin{array}{l}\text { Jaurens (C) (pt.p) } \\
\text { (Mourer-Chauviré, 1980) }\end{array}$ & & $\begin{array}{c}20 \\
43,2\end{array}$ & $\begin{array}{c}16 \\
47,8\end{array}$ & $\begin{array}{c}36 \\
45,5\end{array}$ & $\begin{array}{c}17 \\
54,2\end{array}$ & $\begin{array}{c}22 \\
53,7\end{array}$ & $\begin{array}{c}39 \\
53,9\end{array}$ & $\begin{array}{c}19 \\
48,8\end{array}$ \\
\hline $\begin{array}{l}\text { Solutré Magd. (D) (pt.p) } \\
\text { (Guadelli, 1991) }\end{array}$ & $\begin{array}{c}8 \\
25,0\end{array}$ & $\begin{array}{c}18 \\
45,8\end{array}$ & $\begin{array}{c}26 \\
50,8\end{array}$ & $\begin{array}{c}44 \\
48,8\end{array}$ & $\begin{array}{c}31 \\
52,9\end{array}$ & $\begin{array}{c}26 \\
57,2\end{array}$ & $\begin{array}{c}62 \\
54,9\end{array}$ & 28 \\
\hline
\end{tabular}

Tableau 5 : Valeurs de l'indice protoconique chez différents Equidés 


\begin{tabular}{|l|c|c|c|c|}
\hline & $\mathrm{I}_{1}$ & $\mathrm{I}_{2}$ & $\mathrm{I}_{3}$ & Canine ini. \\
\hline DMDo & $\begin{array}{c}5 \\
15,7-21,0 \\
18,1\end{array}$ & $\begin{array}{c}17,5-22,0 \\
19,8\end{array}$ & $\begin{array}{c}2 \\
17,5-17,7\end{array}$ & $\begin{array}{c}2 \\
2\end{array}$ \\
\hline DVLo & $\begin{array}{c}5 \\
9,6-13,6 \\
11,2\end{array}$ & $\begin{array}{c}10,0-11,5 \\
4\end{array}$ & 11,1
\end{tabular}

Tableau 6: Camp-de-Peyre - Dimensions des incisives et canines inférieures

\begin{tabular}{|l|c|c|c|}
\hline & $\mathrm{P}_{2}-\mathrm{M}_{3}$ & $\mathrm{P}_{2}-\mathrm{P}_{4}$ & $\mathrm{M}_{1}-\mathrm{M}_{3}$ \\
\hline \multirow{2}{*}{ DMDo } & 2 & 3 & 3 \\
& $196,0-197,0$ & $101,0-$ & $93,0-95,5$ \\
& & $102,0-103,5$ & $(94,2)$ \\
\hline \multirow{2}{*}{ DMDalv. } & & 2 & 1 \\
\hline
\end{tabular}

Tableau 7 : Camp-de-Peyre - Dimensions des séries dentaires inférieures

\begin{tabular}{|c|c|c|c|c|}
\hline & $\mathrm{P}_{2}$ & $\mathrm{P}_{3}$ & $\mathrm{P}_{4}$ & $\mathrm{P}_{3}-4$ \\
\hline DMDo & $\begin{array}{c}7 \\
34,5-40,0 \\
37,4 \pm 1,6\end{array}$ & $\begin{array}{c}6 \\
31,0-34,4 \\
33,4 \pm 1,0\end{array}$ & $\begin{array}{c}5 \\
31.5-34.5 \\
33,4\end{array}$ & $\begin{array}{c}31.0-34.5 \\
33.4 \pm 0.6\end{array}$ \\
\hline DVLo & $\begin{array}{c}6 \\
15,9-17,2 \\
16,4 \pm 0,4\end{array}$ & $\begin{array}{c}4 \\
18,0-20,0 \\
19,1\end{array}$ & $\begin{array}{c}5 \\
19,0-20,3 \\
19,7\end{array}$ & $\begin{array}{c}9 \\
18.0-20.3 \\
19.4 \pm 0,4\end{array}$ \\
\hline DMDpF & $\begin{array}{c}4 \\
10,5-20,0 \\
14,9\end{array}$ & $\begin{array}{c}3 \\
10,0-18,0 \\
(15,3)\end{array}$ & $\begin{array}{c}3 \\
15,2-17,0 \\
(16.2)\end{array}$ & $\begin{array}{c}6 \\
10,0-18.0 \\
15.8 \pm 2.5\end{array}$ \\
\hline IF & $\begin{array}{c}4 \\
30,0-53,3 \\
40,65\end{array}$ & $\begin{array}{c}3 \\
32,3-52,9 \\
(46,0)\end{array}$ & $\begin{array}{c}3 \\
45,4-52,4 \\
(49,1)\end{array}$ & $\begin{array}{c}6 \\
32.3-52,9 \\
47,6 \pm 6,6\end{array}$ \\
\hline DMDp & $\begin{array}{c}3 \\
36,0-37,0 \\
(36,3)\end{array}$ & $\begin{array}{c}4 \\
32,1-33,3 \\
32,6\end{array}$ & $\frac{2}{2}$ & $\begin{array}{c}7 \\
31.0-33.3 \\
32.1 \pm 0.6\end{array}$ \\
\hline DVLp & $\begin{array}{c}3 \\
16,0-17,0 \\
(16,6)\end{array}$ & $\begin{array}{c}4 \\
18,5-20,0 \\
19,1\end{array}$ & $\frac{2}{2}$ & $\begin{array}{c}7 \\
18,5-21.8 \\
19,3 \pm 0,9\end{array}$ \\
\hline
\end{tabular}

Tableau 8: Camp-de-Peyre - Dimensions des prémolaires inférieures

\begin{tabular}{|l|c|c|c|c|}
\hline & $\mathrm{M}_{1}$ & $\mathrm{M}_{2}$ & $\mathrm{M}_{1-2}$ & $\mathrm{M}_{3}$ \\
\hline \multirow{2}{*}{ DMDo } & 5 & 8 & 13 & 7 \\
& $28,7-31,0$ & $29,0-31,7$ & $28,7-31,7$ & $33,0-38,0$ \\
& 30,1 & $30,7 \pm 0,6$ & $30,5 \pm 0,4$ & $35,9 \pm 1,1$ \\
\hline \multirow{2}{*}{ DVLo } & 5 & 5 & 10 & 5 \\
& $17,4-18,5$ & $17,0-18,5$ & $17,0-18,5$ & $13,6-15,7$ \\
& 17,8 & 17,8 & $17,8 \pm 0,3$ & 14,9 \\
\hline \multirow{2}{*}{ DMDpF } & 3 & 4 & 7 & \\
& $11,4-12,8$ & $11,5-12,2$ & $11,4-12,8$ & \\
& $(12,0)$ & 11,9 & $11,9 \pm 0,4$ & \\
\hline \multirow{2}{*}{ IF } & 3 & 4 & 7 & \\
& $39,6-42,7$ & $37,5-42,1$ & $37,5-42,7$ & \\
\hline \multirow{2}{*}{ DMIp } & $(40,7)$ & 39,3 & $39,9 \pm 1,4$ & \\
& 4 & 6 & 10 & 5 \\
& $27,4-28,0$ & $26,9-31,0$ & $26,9-31,0$ & $35,0-38,7$ \\
\hline \multirow{2}{*}{ DVLp } & 27,7 & $28,9 \pm 1,3$ & $28,4 \pm 0,8$ & 37,8 \\
\hline & 4 & 6 & 10 & 5 \\
& $16,5-18,2$ & $15,5-17,8$ & $15,5-18,2$ & $14,0-15,0$ \\
& 17,3 & $16,6 \pm 0,6$ & $16,9 \pm 0,5$ & 14,8 \\
\hline
\end{tabular}

Tableau 9: (amp-de-Peyre - Dimensions des molaires inférieures

\begin{tabular}{|l|c|c|c|c|}
\hline & $\mathrm{D}^{2}$ & $\mathrm{D}^{3}$ & $\mathrm{D}^{4}$ & $\mathrm{D}^{3-4}$ \\
\hline \multirow{2}{*}{ DMDo } & 3 & 6 & 6 & 12 \\
& $44,0-46,3$ & $32,0-33,4$ & $32,5-36,7$ & $32,0-36,7$ \\
& $(44,8)$ & $32,5 \pm 0,4$ & $34,1 \pm 1,3$ & $33,3 \pm 0,7$ \\
\hline \multirow{2}{*}{ DVLo } & 2 & 5 & 5 & 10 \\
& $25,0-26,0$ & $25,0-27,4$ & $25,0-26,4$ & $25,0-27,4$ \\
& & 25,8 & 25,8 & $25,8 \pm 0,4$ \\
\hline \multirow{2}{*}{ DMDpr.o } & 2 & 6 & 6 & 12 \\
& $9,0-9,1$ & $9,7-11,0$ & $11,7-13,7$ & $9,7-13,7$ \\
& & $10,3 \pm 0,5$ & $12,2 \pm 0,7$ & $11,3 \pm 0,6$ \\
\hline \multirow{2}{*}{ IPo } & 2 & 6 & 6 & 12 \\
& $19,7-20,5$ & $30,3-34,0$ & $33,5-38,5$ & $30,3-38,5$ \\
& & $31,7 \pm 1,2$ & $35,8 \pm 1,6$ & $33,8 \pm 1,4$ \\
\hline
\end{tabular}

Tableau 10 : Canı-de-Peyre - Dimensions des dents déciduales supérieures

\begin{tabular}{|l|c|c|c|c|}
\hline & $\mathrm{D}_{2}$ & $\mathrm{D}_{3}$ & $\mathrm{D}_{4}$ & $\mathrm{D} 3-4$ \\
\hline DMDo & 2 & 3 & 3 & 8 \\
& $39,1-39,3$ & $\begin{array}{c}33,3-34,0 \\
(33,6)\end{array}$ & $\begin{array}{c}35,6-38,8 \\
(37,0)\end{array}$ & $\begin{array}{c}32,2-38,8 \\
34,7 \pm 1,4\end{array}$ \\
\hline \multirow{2}{*}{ DVLo } & 2 & 3 & 3 & 8 \\
& $11,0-14,0$ & $\begin{array}{c}14,8-14,8 \\
(14,8)\end{array}$ & $\begin{array}{c}13,4-14,4 \\
(13,9)\end{array}$ & $\begin{array}{c}13,4-16,0 \\
14,7 \pm 0,6\end{array}$ \\
\hline \multirow{2}{*}{ DMDpF } & 2 & 3 & 3 & 8 \\
& $18,0-19,9$ & $\begin{array}{c}14,8-15,2 \\
(15,1)\end{array}$ & $\begin{array}{c}15,5-15,5- \\
15,8\end{array}$ & $\begin{array}{c}10,5-16,5 \\
14,9 \pm 1,2\end{array}$ \\
\hline \multirow{2}{*}{ IF } & 2 & 3 & 3 & 8 \\
& $38,4-45,8$ & $\begin{array}{c}44,1-44,7- \\
45,7\end{array}$ & $\begin{array}{c}40,0-43,2- \\
43,5\end{array}$ & $\begin{array}{c}31,3-51,2 \\
43,0 \pm 3,8\end{array}$ \\
\hline
\end{tabular}




\begin{tabular}{|c|c|}
\hline DT minimum du col & $\stackrel{2}{6}$ \\
\hline $\begin{array}{l}\text { DAP maximum extrémité } \\
\text { inférieure }\end{array}$ & $\begin{array}{c}3 \\
102,0-103,0 \\
(102,3)\end{array}$ \\
\hline DAP cavité glénoïde & $\begin{array}{c}5 \\
60,0-66,5 \\
63,4 \pm 2,4 \\
\end{array}$ \\
\hline DT cavité glénoìde & $\begin{array}{c}5 \\
51,0-56,0 \\
54,2 \pm 2,0\end{array}$ \\
\hline
\end{tabular}

Tableau 12: Camp-de-Peyre - Dimensions des scapulums

\begin{tabular}{|l|c|}
\hline Hauteur maximum & 1 \\
\hline DT maximumn proximal & 410,5 \\
\hline DAP maximum proximal & $100,5-114,0$ \\
& 107,8 \\
\hline DT minimum diaphyse & $108,0-114,0$ \\
\hline & 111,1 \\
\hline DT maximum distal & $37,0-37,5$ \\
\hline DT articulaire distal & 3 \\
& $89,5-94,0$ \\
& 92,2 \\
\hline DAP maximum distal & 6 \\
& $84,0-87,0$ \\
& $84,9 \pm 1,1$ \\
\hline DAP distal & 6 \\
& $91,5-98,0$ \\
& $95,4 \pm 1,9$ \\
\hline
\end{tabular}

\begin{tabular}{|l|c|}
\hline Hauteur de l'os radio-unaire & 436,0 \\
\hline Hauteur du radius & 3 \\
& $347,0-360,0$ \\
$(352,8)$ \\
\hline DT maximum proximal du radius & 5 \\
& $93,0-96,0$ \\
& $94,4 \pm 1,1$ \\
\hline DT articulaire proximal du radius & 4 \\
& $85,0-86,0$ \\
& 85,4 \\
\hline DAP maximum proximal du & 9 \\
radius & $51,0-55,0$ \\
\hline DAP articulaire proximal du & $43,1 \pm 0,8$ \\
radius & $44,8 \pm 1,0$ \\
\hline DT milieu diaphyse du radius & 3 \\
& $46,0-46,5$ \\
& $(46,2)$ \\
\hline DT maximum distal & 9 \\
& $83,0-90,5$ \\
& $86,1 \pm 1,0$ \\
\hline DT articulaire distal du radius & 10 \\
& $70,5-74,5$ \\
& $72,8 \pm 0,8$ \\
\hline DAP maximum distal & 10 \\
& $49,0-55,0$ \\
& $51,7 \pm 1,3$ \\
\hline DAP distal & 12 \\
& $39,5-44,5$ \\
& $42,2 \pm 0,9$ \\
\hline
\end{tabular}

Tableau 14 : Camp-de-Peyre - Dimensions des os radio-ulnaires 


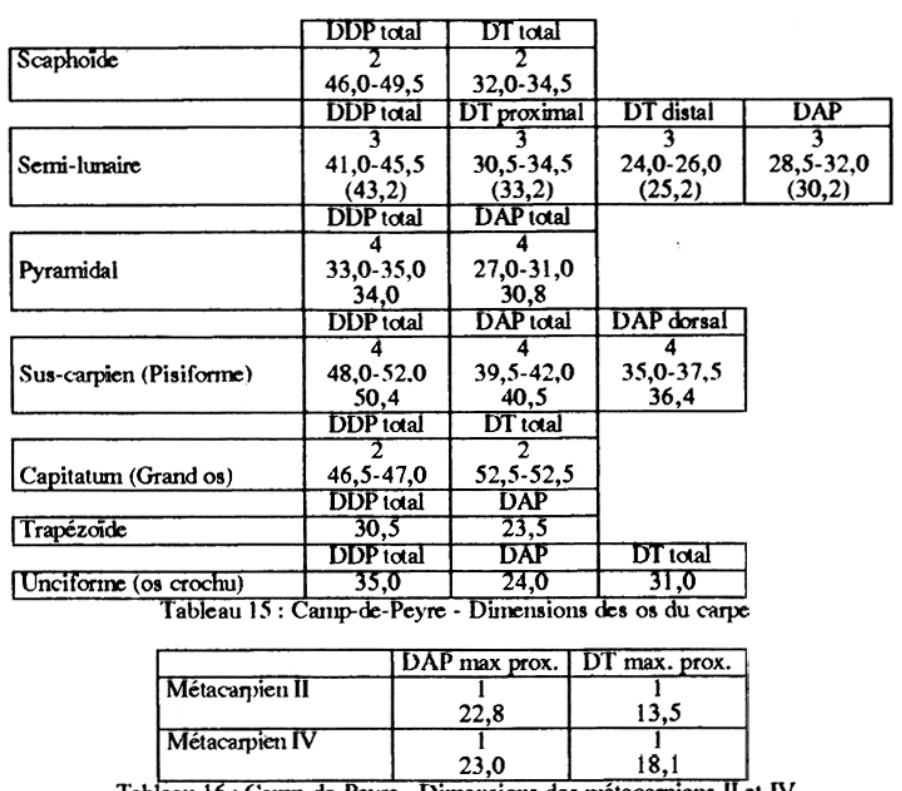

Tableau 16: C'amp-de-Peyre - Dimensions des métacarpiens II et IV

\begin{tabular}{|c|c|c|c|}
\hline Métacarpiens III & $\begin{array}{l}\text { Camp-de- } \\
\text { Peyre }\end{array}$ & $\begin{array}{c}\text { Mosbach } \\
\text { (Eisenmann, 79) }\end{array}$ & $\begin{array}{l}\text { Lunel-Viel } \\
\text { (Bonifay,80) }\end{array}$ \\
\hline 1:Hauteur totale & $\begin{array}{c}4 \\
238,5-248,0 \\
242,6\end{array}$ & $\begin{array}{c}39 \\
246,0-271,5 \\
261,0\end{array}$ & $\begin{array}{c}13 \\
222,0-241,7 \\
232,85\end{array}$ \\
\hline 2:Hauteur externe & $\begin{array}{c}4 \\
235,0-244,0 \\
238,7\end{array}$ & $\begin{array}{c}27 \\
240,0-261,5 \\
250,9\end{array}$ & \\
\hline 3:DT milieu diaphyse & $\begin{array}{c}4 \\
36,5-39,5 \\
37,4\end{array}$ & $\begin{array}{c}53 \\
37,0-47,5 \\
42,2\end{array}$ & $\begin{array}{c}13 \\
36,1-44,4 \\
40,05\end{array}$ \\
\hline $\begin{array}{l}\text { 4:DAP au milieu de la } \\
\text { diaphyse }\end{array}$ & $\frac{2}{28,0-29,8}$ & $\begin{array}{c}54 \\
27,5-35,5 \\
30,9\end{array}$ & \\
\hline $\begin{array}{l}\text { 5:DT articulaire } \\
\text { proximal }\end{array}$ & $\begin{array}{c}4 \\
50,5--56,5 \\
54,1\end{array}$ & $\begin{array}{c}39 \\
55,0-62,0 \\
58,2\end{array}$ & \\
\hline $\begin{array}{l}5 ' \text { 'DT maximum } \\
\text { proximal }\end{array}$ & $\begin{array}{c}5 \\
52,-56,5 \\
53,8 \pm 1,6\end{array}$ & & $\begin{array}{c}12 \\
50,0-62,6 \\
53,34\end{array}$ \\
\hline $\begin{array}{l}\text { 6:DAP articulaire } \\
\text { proximal }\end{array}$ & $\begin{array}{c}5 \\
33,5-37,5 \\
35,6 \pm 1,4\end{array}$ & $\begin{array}{c}40 \\
35,0-40,5 \\
37,9\end{array}$ & \\
\hline $\begin{array}{l}\text { 6':I)AP maximum } \\
\text { proximal }\end{array}$ & $\begin{array}{c}6 \\
35,0-39,5 \\
37,8 \pm 1,3\end{array}$ & & $\begin{array}{c}12 \\
31,1-39,5 \\
36,59\end{array}$ \\
\hline $\begin{array}{l}\text { 7:Diamètre de la } \\
\text { facette répondant au. } \\
\text { magmum }\end{array}$ & $\begin{array}{c}4 \\
40,5-47,0 \\
43,6\end{array}$ & $\begin{array}{c}40 \\
43,0-50,5 \\
46,8\end{array}$ & \\
\hline $\begin{array}{l}\text { 8:DT facette } \\
\text { antérieure répondant à } \\
\text { l'unciforme }\end{array}$ & $\begin{array}{c}4 \\
15,8-19,3 \\
17,9\end{array}$ & $\begin{array}{c}40 \\
14,0-20,5 \\
17,2\end{array}$ & \\
\hline $\begin{array}{l}\text { 8:DT facette } \\
\text { postérieure répondant } \\
\text { à l'unciforme }\end{array}$ & $\begin{array}{c}4 \\
6,9-9,6 \\
7,8\end{array}$ & $\begin{array}{c}24 \\
7,5-13,0 \\
9,7\end{array}$ & \\
\hline $\begin{array}{l}\text { 9:DT facette } \\
\text { répondant au } \\
\text { trapézoide }\end{array}$ & 6,0 & $\begin{array}{c}19 \\
0-9,0 \\
5,3\end{array}$ & \\
\hline $\begin{array}{l}\text { 10:DT sus-articulaire } \\
\text { distal }\end{array}$ & $\begin{array}{c}6 \\
51,0-58,0 \\
54,4 \pm 2,2\end{array}$ & $\begin{array}{c}29 \\
52,5-59,5 \\
56,5\end{array}$ & $\begin{array}{c}14 \\
48,2-58,3 \\
51,98\end{array}$ \\
\hline $\begin{array}{l}\text { 11:DT articulaire } \\
\text { distal }\end{array}$ & $\begin{array}{c}5 \\
51,0-56,0 \\
54,4 \pm 2,2\end{array}$ & $\begin{array}{c}27 \\
51,5-59,0 \\
56,5\end{array}$ & $\begin{array}{c}14 \\
50,2-57,3 \\
52,10\end{array}$ \\
\hline $\begin{array}{l}\text { 12:DAP articulaire } \\
\text { distal }\end{array}$ & $\begin{array}{c}4 \\
38,5-43,3 \\
40,3\end{array}$ & $\begin{array}{c}23 \\
39,0-46,5 \\
42,9\end{array}$ & $\begin{array}{c}13 \\
37,0-42,3 \\
40,04 \\
\end{array}$ \\
\hline $\begin{array}{l}\text { 12':DAP sus- } \\
\text { articulaire distal }\end{array}$ & $\begin{array}{c}5 \\
25,5-28,0 \\
26,6 \pm 1,0\end{array}$ & & \\
\hline $\begin{array}{l}\text { 13:DAP minimum du } \\
\text { condyle médial distal }\end{array}$ & $\begin{array}{c}4 \\
31,5-33,5 \\
32,9 \\
\end{array}$ & $\begin{array}{c}25 \\
31,0-37,0 \\
34,5\end{array}$ & \\
\hline
\end{tabular}




\begin{tabular}{|c|c|c|c|}
\hline $\begin{array}{l}\text { 14:DAP maximum du } \\
\text { condyle médial distal }\end{array}$ & $\begin{array}{c}4 \\
32,3-34,5 \\
34,0\end{array}$ & $\begin{array}{c}22 \\
32,5-39,5 \\
36,3\end{array}$ & \\
\hline IR $(3 / 1)$ & $\begin{array}{c}4 \\
14,72-16,56 \\
15,41\end{array}$ & & $\begin{array}{c}11 \\
15,8-18,6 \\
17,06\end{array}$ \\
\hline $10 / 11$ & $\begin{array}{c}5 \\
93,8-101,8 \\
98,76 \pm 3,1\end{array}$ & & $\begin{array}{c}12 \\
94-104 \\
99,08\end{array}$ \\
\hline 6.15 & $\begin{array}{c}5 \\
66,7-73.3 \\
69,7 \pm 2,4\end{array}$ & & \\
\hline $6 \% / 5$ & $\begin{array}{c}4 \\
68,14-70,27 \\
69,29\end{array}$ & & \\
\hline $6 / 5^{\prime}$ & $\begin{array}{c}4 \\
63,72-68,57 \\
65,08\end{array}$ & & \\
\hline $6 / 5$ & $\begin{array}{c}4 \\
63,72-66,34 \\
64,93\end{array}$ & & \\
\hline
\end{tabular}

\begin{tabular}{|c|c|c|c|}
\hline lère phalange antérieure & & 2ème phalange antérieure & \\
\hline Hauteur totale & $\begin{array}{c}5 \\
88,0-94,0 \\
91,1 \pm 2,6\end{array}$ & Hauteur totale & $\begin{array}{c}5 \\
50,0-52,0 \\
50,9 \\
\pm 0,7\end{array}$ \\
\hline Hauteur antérieure & $\begin{array}{c}5 \\
80,5-85,5 \\
82,0 \pm 2,0 \\
\end{array}$ & DT maximum proximal & $\begin{array}{c}4 \\
56,5-61,5 \\
58,9\end{array}$ \\
\hline DT maximum proximal & 60,5 & DT articulaire proximal & $\begin{array}{c}4 \\
51,0-57,5 \\
54,0\end{array}$ \\
\hline DT articulaine proximal & $\begin{array}{c}3 \\
53,0-57,0 \\
(55,2) \\
\end{array}$ & DAP proximal & $\begin{array}{c}4 \\
33,5-36,5 \\
35,0 \\
\end{array}$ \\
\hline DAP proximal & 41,0 & DT minimum & $\begin{array}{c}5 \\
48,5-53,5 \\
50,6 \pm 1,9\end{array}$ \\
\hline DT au milieu & $\begin{array}{c}5 \\
37,0-41,0 \\
39,2 \pm 1,4\end{array}$ & DT maximum distal & $\begin{array}{c}3 \\
55,0-57,5 \\
56,3\end{array}$ \\
\hline DT sus-articulaire distal & $\begin{array}{c}5 \\
50,0-53,0 \\
50,8 \pm 1,2\end{array}$ & DAP maximum distal & $\begin{array}{c}3 \\
27,5-29,5 \\
28,7 \\
\end{array}$ \\
\hline DT articulaire distal & $\begin{array}{c}4 \\
47,0-52,0 \\
49,8\end{array}$ & $\begin{array}{l}\text { IR (DT mini / Hauteur } \\
\text { totale) }\end{array}$ & $\begin{array}{c}5 \\
93,3-104,9 \\
99,4 \pm 4,2\end{array}$ \\
\hline IR (DT milieu/Haut. totale) & $\begin{array}{c}5 \\
40,4-45,2 \\
43,1 \pm 1,9\end{array}$ & & \\
\hline $\begin{array}{l}\text { DT art. dist./DT sus-art. } \\
\text { dist. }\end{array}$ & $\begin{array}{c}4 \\
94,0-103,0 \\
99,0\end{array}$ & & \\
\hline
\end{tabular}

Tableau 18: Camp-de-Peyre - Dimensions des lère et 2ème phalanges antérieures

\begin{tabular}{|l|c|}
\hline DAP articulaire proximal & 2 \\
\hline DT maximum distal & $66,0-67,0$ \\
\hline DT maximum de la trochlée & 103,0 \\
& 4 \\
& $102,0-109,0$ \\
& 105,3 \\
\hline
\end{tabular}

\begin{tabular}{|l|c|}
\hline DT total & 76,0 \\
\hline DAP total & 78,0 \\
\hline
\end{tabular}

Tableau 20: Camp-de-Peyre - Dimensions de la rotule (patella) 


\begin{tabular}{|c|c|}
\hline Hauteur totale & $\begin{array}{c}3 \\
367,0-388.5 \\
(379,7) .\end{array}$ \\
\hline DT proximal & $\begin{array}{c}4 \\
106,5-113,0 \\
110,1\end{array}$ \\
\hline DAP proximal & $\begin{array}{c}3 \\
95,0-99,0 \\
(96,7)\end{array}$ \\
\hline DT minimum de la diaptyse & $\begin{array}{c}3 \\
52,0-53,5 \\
(52,7)\end{array}$ \\
\hline DT distal & $\begin{array}{c}9 \\
84,0-90,0 \\
87,2 \pm 1,1 \\
\end{array}$ \\
\hline DAP distal & $\begin{array}{c}9 \\
50,0-53,5 \\
52,2 \pm 0,8\end{array}$ \\
\hline $\begin{array}{l}\text { IR (DT mini. diap./Haut. } \\
\text { tot.) }\end{array}$ & $\begin{array}{c}3 \\
13,6-14,3 \\
(13,8)\end{array}$ \\
\hline
\end{tabular}

\begin{tabular}{|c|c|c|c|c|c|}
\hline & Hauteur & $\begin{array}{l}\text { DT entre les } \\
\text { lèves de la } \\
\text { troclulie }\end{array}$ & $\begin{array}{c}\text { DT } \\
\text { articulaine } \\
\text { distal }\end{array}$ & $\begin{array}{c}\text { DDP } \\
\text { articulaire } \\
\text { distal }\end{array}$ & \\
\hline \multirow[t]{2}{*}{ Takus } & $\begin{array}{c}5 \\
65.5-69,0 \\
66,6 \pm 1,3\end{array}$ & $\begin{array}{c}4 \\
49,0-52,0 \\
50,1\end{array}$ & $\begin{array}{c}4 \\
60,5-61,0 \\
60,9\end{array}$ & $\begin{array}{c}3 \\
40,0-41,0 \\
(40,7)\end{array}$ & \\
\hline & Hauteur & $\begin{array}{l}\text { DT max. } \\
\text { tub. prox. }\end{array}$ & $\begin{array}{l}\text { DDP max. } \\
\text { tub. prox. }\end{array}$ & $\begin{array}{l}\text { DDP mini. } \\
\text { tub. prox. }\end{array}$ & $\begin{array}{c}\text { DDP niv. } \\
\text { bec }\end{array}$ \\
\hline \multirow[t]{2}{*}{ Calcanéum } & $\begin{array}{c}121,0131,0 \\
124,7 \pm 4,1 \\
\end{array}$ & $\begin{array}{c}4 \\
37,0-41,0 \\
39,1\end{array}$ & $\begin{array}{r}5 \\
53,5-60,5 \\
56,8 \pm 2,9 \\
\end{array}$ & $\begin{array}{c}46,0-54,0 \\
50,4 \\
\end{array}$ & $\begin{array}{r}5 \\
55,5-60,0 \\
57,5 \pm 1,8 \\
\end{array}$ \\
\hline & DDPtoal & DTioal & & & \\
\hline \multirow[t]{2}{*}{ Naviculaire } & $\begin{array}{c}3 \\
47,5-50,0 \\
48,5\end{array}$ & $\begin{array}{c}3 \\
26,0-59,0 \\
54,5\end{array}$ & & & \\
\hline & DDPtoal & IT toal & & & \\
\hline \multirow[t]{2}{*}{ Cuboide } & $\begin{array}{c}3 \\
41.5-43,0 \\
42,3\end{array}$ & $\begin{array}{c}3 \\
24,0-27,0 \\
25,3\end{array}$ & & & \\
\hline & DDPtoal & DT total & & & \\
\hline $\begin{array}{l}\text { Grand } \\
\text { cunćiforme }\end{array}$ & $\begin{array}{c}3 \\
47,5-50,0 \\
48,7\end{array}$ & $\begin{array}{c}3 \\
53,0-55,5 \\
54,7\end{array}$ & & & \\
\hline
\end{tabular}

\begin{tabular}{|l|c|}
\hline DAP max prox. & 23,5 \\
\hline DT max. prox. & 14,3 \\
\hline
\end{tabular}

Tableau 23 : Camp-de-Peyre - Dimensions du métatarsien II

\begin{tabular}{|c|c|c|c|c|c|}
\hline Metatarsiens III & $\begin{array}{l}\text { Canmp-de- } \\
\text { Pesre }\end{array}$ & $\begin{array}{c}\text { Mosbach } \\
\text { (Eisenmann, 79) }\end{array}$ & \begin{tabular}{|l|} 
Linel-Viel \\
(niv. inf.) \\
(Ronifay,80)
\end{tabular} & \begin{tabular}{|c|} 
Chátillon-St Jean \\
(Mourer- \\
Chauviré,72)
\end{tabular} & $\begin{array}{l}\text { Coupe-Gorge } \\
\text { (Guadelli,90) }\end{array}$ \\
\hline 1:Hauteur totale & \begin{tabular}{|c|}
6 \\
$284,5-301,5$ \\
$288,6 \pm 5,3$ \\
\end{tabular} & $\begin{array}{c}42 \\
292,0-322,0 \\
308,5\end{array}$ & $\begin{array}{c}9 \\
269,0-286,0\end{array}$ & $\begin{array}{c}17 \\
285,9 \pm 4,57\end{array}$ & $\begin{array}{c}3 \\
286,0-306,0 \\
(294,7)\end{array}$ \\
\hline 3:DT milieu de la diaphyse & \begin{tabular}{|c|}
6 \\
$36,0-39,0$ \\
$37,9 \pm 0,8$ \\
\end{tabular} & $\begin{array}{c}62 \\
37,5-44,5 \\
41,0\end{array}$ & $\begin{array}{c}13 \\
36,3-44,4 \\
40,11 \\
\end{array}$ & $\begin{array}{c}17 \\
37,8 \pm 1,82\end{array}$ & $\begin{array}{c}38,0-43,0 \\
(36,7)\end{array}$ \\
\hline $\begin{array}{l}\text { 4:DAP milieu de la } \\
\text { diaplyse }\end{array}$ & $\begin{array}{c}5 \\
34,9-37,5 \\
35,9\end{array}$ & $\begin{array}{c}62 \\
34,5-41,5 \\
38,2\end{array}$ & & & $\begin{array}{c}3 \\
35,5-40.8 \\
(38,7)\end{array}$ \\
\hline S:DT maximum proximal & $\begin{array}{c}6 \\
55,0-60,0 \\
58,2 \pm 1,7\end{array}$ & $\begin{array}{c}49 \\
56,0 \cdot 64,0 \\
59,7\end{array}$ & 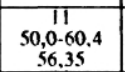 & $\begin{array}{c}17 \\
56,9 \pm 1,61\end{array}$ & $\begin{array}{c}3 \\
54,0-60,0 \\
(57,3)\end{array}$ \\
\hline 6:DAP maximum proximal & $\begin{array}{c}6 \\
48.5-54.5 \\
51,6 \pm 1,7\end{array}$ & $\begin{array}{c}49 \\
46,0-56,0 \\
50,1\end{array}$ & $\begin{array}{c}11 \\
44,2-53,4 \\
48,03\end{array}$ & & $\begin{array}{c}3 \\
47,0-53,5 \\
(51,1)\end{array}$ \\
\hline \begin{tabular}{|l|l|}
$\begin{array}{l}\text { 7:Dianćtre de la facetle } \\
\text { répondant au grand } \\
\text { cunćiforme }\end{array}$ \\
\end{tabular} & $\begin{array}{r}6 \\
49.0-53,0 \\
51,1 \pm 1,3 \\
\end{array}$ & $\begin{array}{c}29 \\
51,0-57,5 \\
53,8\end{array}$ & & & $\begin{array}{c}3 \\
45,0-53,3 \\
(48,9) \\
\end{array}$ \\
\hline $\begin{array}{l}\text { 8:Diamètre de la facette } \\
\text { répondant au cuboide }\end{array}$ & $\begin{array}{c}7 \\
12,7-15,5 \\
14,0 \pm 0,7\end{array}$ & $\begin{array}{c}27 \\
9.0-14,0 \\
11,7\end{array}$ & & & \\
\hline $\begin{array}{l}\text { 9:Diancetre de la facette } \\
\begin{array}{l}\text { répondant aut petit } \\
\text { cunéforme }\end{array}\end{array}$ & 2,9 & $\begin{array}{l}10 \\
0-8 \\
5,3 \\
\end{array}$ & & & \\
\hline 10:IDT sus-articulaire distal & $\begin{array}{c}8 \\
53.0-59.0 \\
55,1 \pm 1,3 \\
\end{array}$ & $\begin{array}{c}53,5 \cdot 61,5 \\
58,3 \\
\end{array}$ & $\begin{array}{c}12 \\
50,2 \cdot 60,5 \\
55,10\end{array}$ & $\begin{array}{c}17 \\
54,9 \pm 1,59 \\
\end{array}$ & $\begin{array}{c}51,2 \cdot 60.5 \\
(56,6) \\
2\end{array}$ \\
\hline 11:DT articulaire distal & $\begin{array}{c}7 \\
53,5-57,5 \\
55,7 \pm 1,1\end{array}$ & $\begin{array}{c}27 \\
53,0-59,5 \\
56,9\end{array}$ & $\begin{array}{c}11 \\
49,5-59,6 \\
53,43\end{array}$ & $\begin{array}{c}17 \\
53,7 \pm 1,06\end{array}$ & $\begin{array}{c}3 \\
52,5-58,2 \\
(55,2)\end{array}$ \\
\hline 12:DAP articulaire distal & $\begin{array}{c}8 \\
40.0 \cdot 44,0 \\
42.1 \pm 1,0\end{array}$ & $\begin{array}{c}27 \\
40.0 \cdot 48,5 \\
43.9\end{array}$ & $\begin{array}{c}12 \\
40,0 \cdot 43,7 \\
41,63\end{array}$ & $41,6 \pm 0,82$ & $\begin{array}{c}40.5-44.0 \\
(42,0)\end{array}$ \\
\hline $\begin{array}{l}\text { 13:DAP nuinimum condyle } \\
\text { nédial distal }\end{array}$ & $\begin{array}{c}8 \\
30,0 \cdot 33,2 \\
31,8 \pm 0,8 \\
\end{array}$ & $\begin{array}{c}30 \\
30.5-38,0 \\
53,6 \\
\end{array}$ & & & $\begin{array}{c}3 \\
30,2-32,5 \\
(31,6)\end{array}$ \\
\hline $\begin{array}{l}\text { 14:1)AP maximum modyle } \\
\text { médial distal }\end{array}$ & $\begin{array}{c}8 \\
31.7-36,7 \\
35,0 \pm 1,2\end{array}$ & $\begin{array}{c}28 \\
32,5-40,0 \\
36,8\end{array}$ & & & $\begin{array}{c}3 \\
29,3-36,2 \\
(33,6)\end{array}$ \\
\hline IR (3/1) & $\begin{array}{c}6 \\
12.6-13,4 \\
13,1 \pm 0,2 \\
\end{array}$ & & \begin{tabular}{|c|}
9 \\
$131.0-152.0$ \\
142,55 \\
\end{tabular} & $\begin{array}{c}17 \\
13,25 \pm 5,28 \\
\end{array}$ & \begin{tabular}{|c|}
3 \\
$12,81 \cdot 14,73$ \\
$(13,61)$
\end{tabular} \\
\hline $10 / 11$ & $\begin{array}{c}6 \\
96,5 \cdot 103,5 \\
99,4 \pm 1,9\end{array}$ & & $\begin{array}{c}1 \\
97,0-107,0 \\
102,81\end{array}$ & $\begin{array}{c}17 \\
102,66 \pm 1,49\end{array}$ & $\begin{array}{c}3 \\
97,5-105,5 \\
(102,31)\end{array}$ \\
\hline
\end{tabular}




\begin{tabular}{|c|c|c|c|}
\hline Tére phalange postérieure & & Leme phalange postéricure & \\
\hline Hauleur totale & 85,0 & Hauteur totale & 54,0 \\
\hline $\begin{array}{l}\text { Hautcur antérieure } \\
\text { D' miax imm proximal }\end{array}$ & $\begin{array}{c}2 \\
62,0-63,0\end{array}$ & $\begin{array}{l}\text { DT maximum proximal } \\
\text { DT articulaire proximal }\end{array}$ & $\begin{array}{r}60,0 \\
51,0\end{array}$ \\
\hline DT articulaire proximal & $\begin{array}{c}2 \\
58,0-58,0\end{array}$ & DAP proximal & 36,0 \\
\hline DAP proximal & $\frac{2}{44,0-44,5}$ & DT minimum & 50,0 \\
\hline DT au milieu & 39,0 & DT maximum distal & 34,0 \\
\hline DT sus-articulaire distal & 49,5 & DAP maximum distal & 30,0 \\
\hline DT arliculaire dista & 48,0 & $\begin{array}{l}\text { IR (OT mint/lauteur } \\
\text { (ctale) }\end{array}$ & 92,59 \\
\hline $\begin{array}{l}\text { IR (DT milieu/lau . totale) } \\
\text { DT art. dist. ITT nus-art. } \\
\text { dist. }\end{array}$ & $\frac{45,88}{96,91}$ & & \\
\hline
\end{tabular}

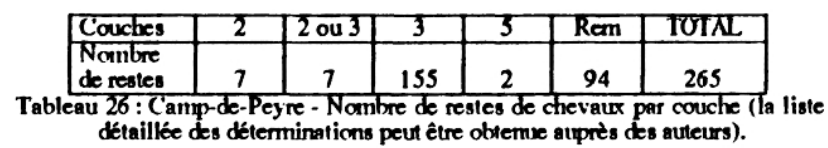

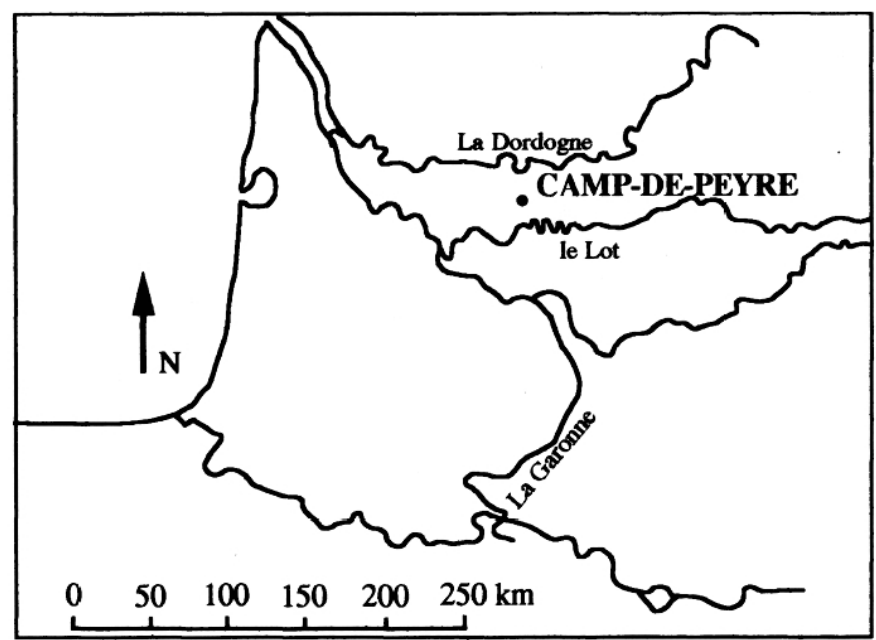

Figure 1 : Carte de localisation géographique du gisement

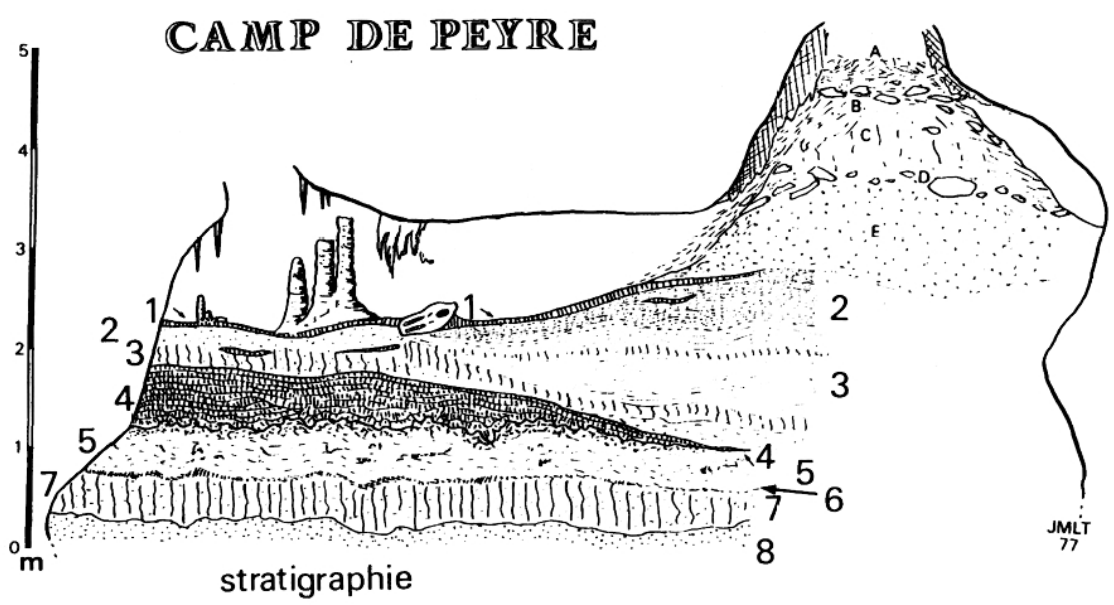

Figure 2 : Stratigraphie de Camp-de-Peyre d'après J.-M. Le Tensorer (1981). Salle dite du Cheval et cône d'éboulis. Au Nord de celui-ci se trouvait la Petite Salle. 


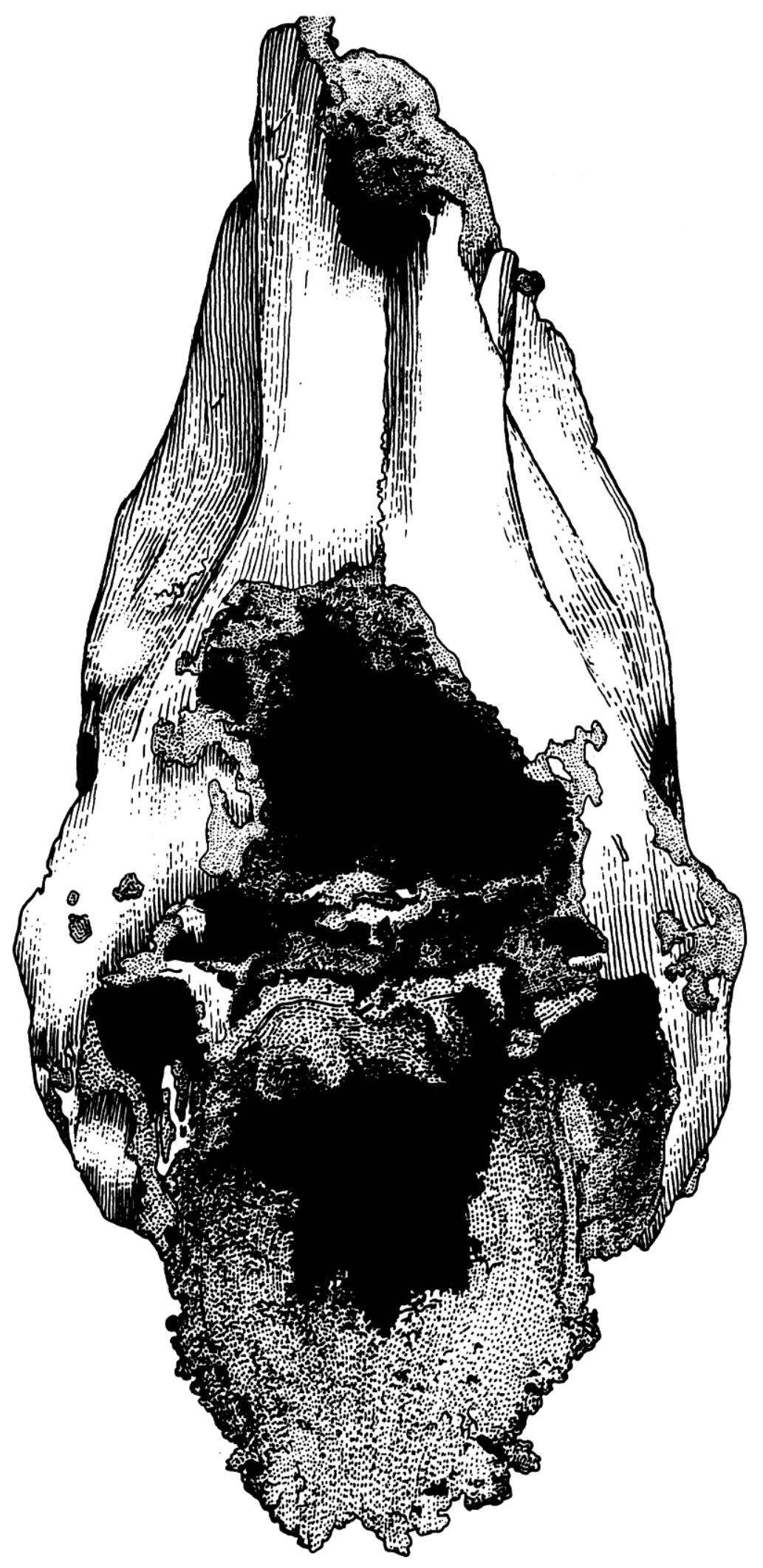

$\frac{2}{3}$

ミ

กิ

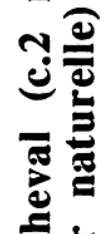

는

을

ํㅜㄹ

苞

نั. 


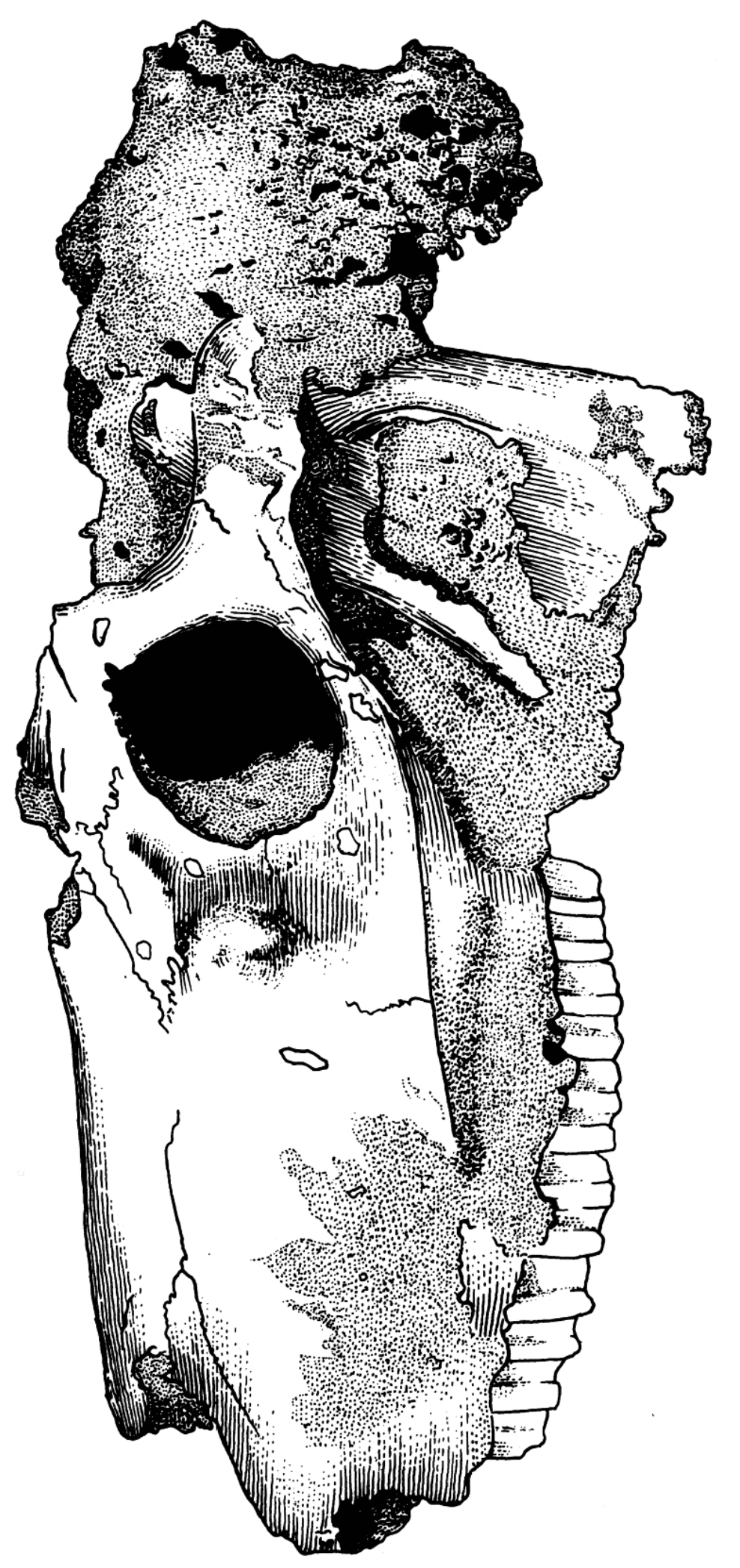

$\frac{5}{3}$

ญัฐ

ָิ

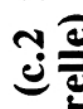

零

ป

월

苞

况

อُ 


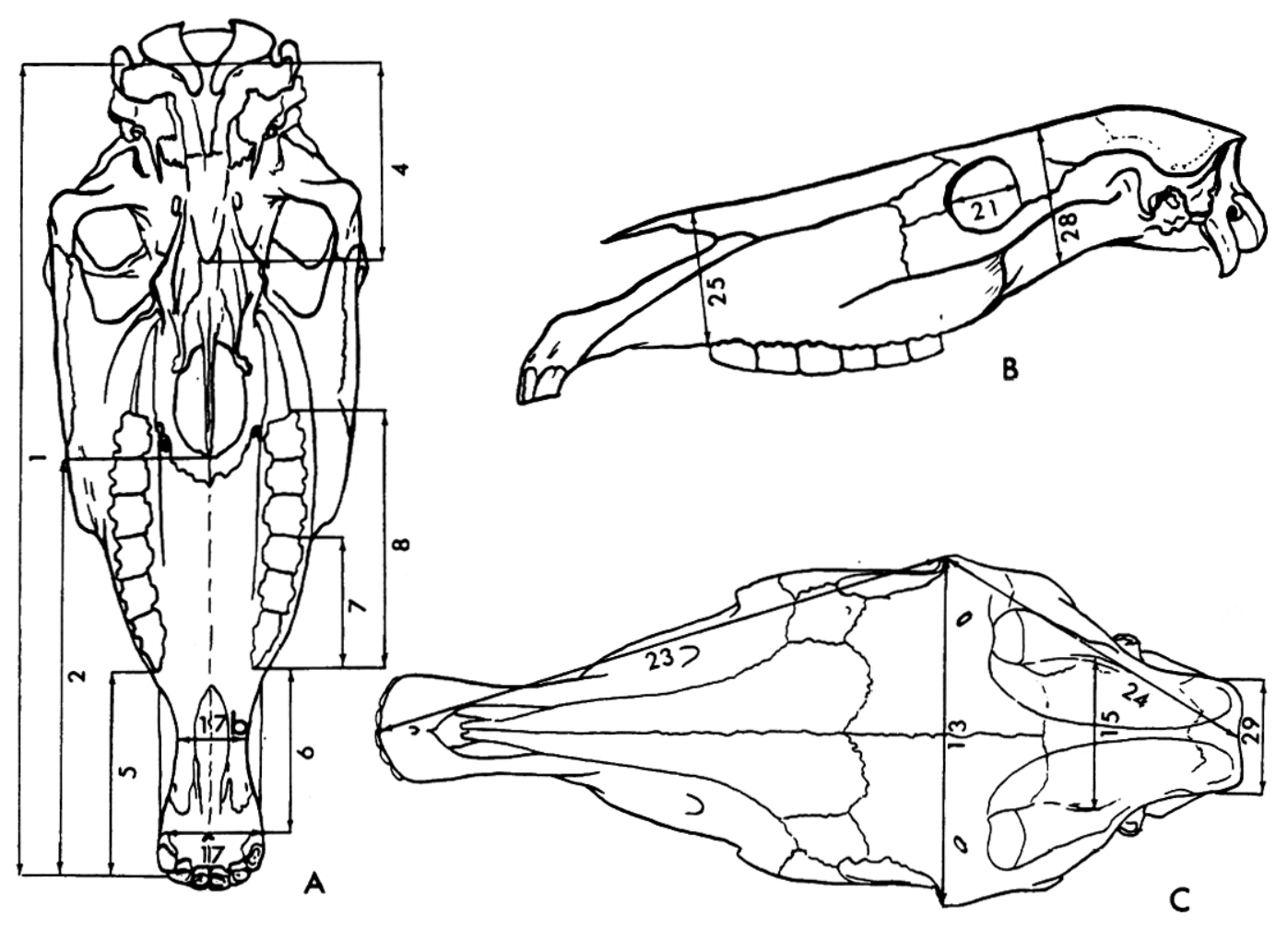

Figure 5 : Equus. Dessins d'après R. Barone (1966).

Mensurations crâniennes d'après V. Eisenmann (1980).

$A$ : vue ventrale, $B$ : vue latérale, $C$ : vue frontale.

1-Longueur basilaire, 2-Longueur palatine, 4-Longueur Vomer/Basion, 5-Longueur du museau, "2-5"-Longueur du palais, 6-Longueur du diastème, 7-Diamètre mésio-distal $\mathrm{P}^{2}-\mathrm{P}^{4}, 8$-Diamètre mésio-distal $\mathrm{P}^{2}-\mathrm{M}^{3}$, 13-Largeur frontale, 15-Largeur maximum de la boîte crânienne, 16Largeur maximum de la protubérance occipitale externe, 17-Largeur du prémaxillaire, 17b-Largeur minimum du museau, 21 Diamètre antéropostérieur de l'orbite, 23-Longueur de la ligne oculaire antérieure, 24Longueur de la ligne oculaire postérieure, 25-Hauteur de la face en avant de $\mathrm{P}^{2}, 28$-Hauteur de la face en arrière des orbites. 


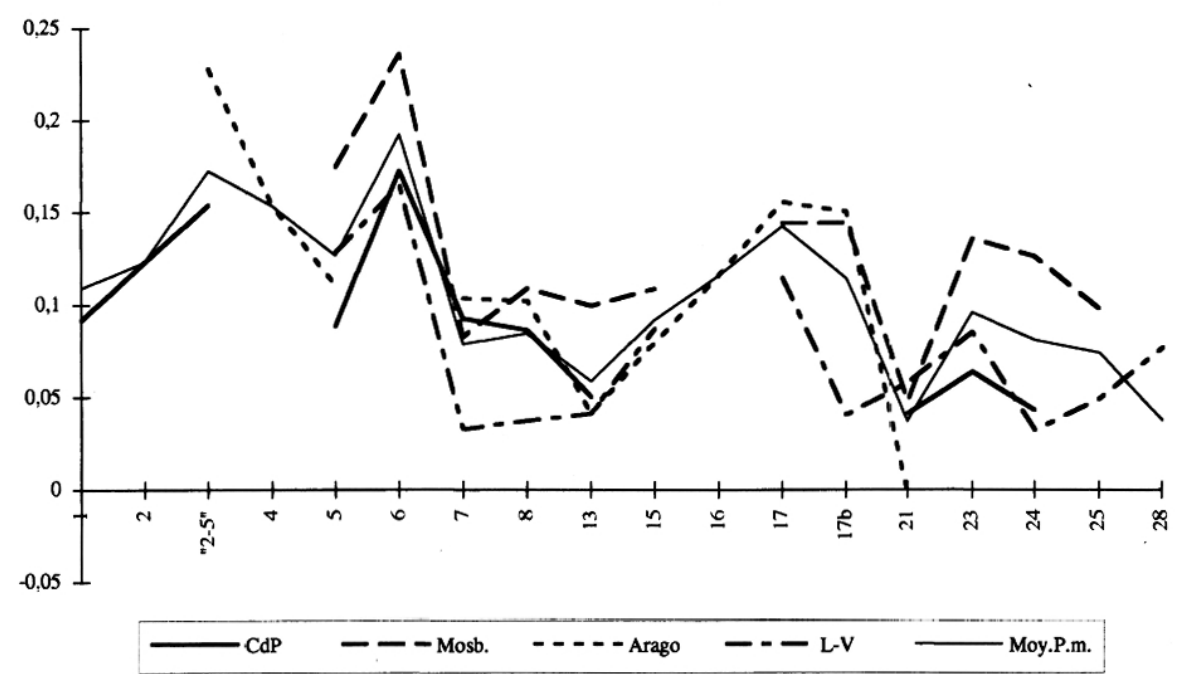

Figure 6 : Comparaison des crânes de chevaux du pléistocène moyen. Diagramme établi selon la méthode de Simpson. En référence (0) : les dimensions moyennes du crâne chez Equus hemionus onager d'après v. Eisenmann et al. 1985.

CdP:Camp-de-Peyre, Mosb:Mosbach, L-V:Lunel-Viel, Moy.P.m.:Moyenne pour le Pléistocène moyen

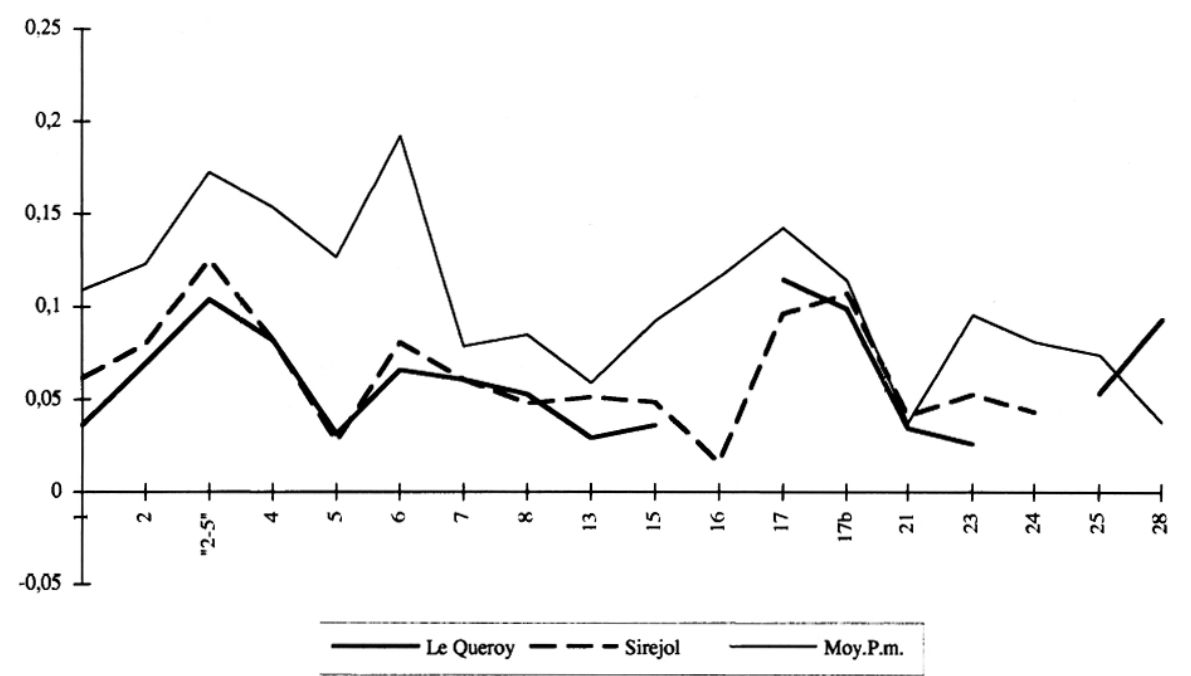

Figure 7 : Comparaison de deux crânes de chevaux du pléistocène supérieur. Diagramme établi selon la méthode de Simpson. En référence $(0)$ : Ies dimensions moyennes du crâne chez Equus hemionus onager d'après V. Eisenmann et al., 1985. Le trait fin indique pour les 3 crânes de chevaux du Pléistocène moyen la valeur "moyenne" de chaque mesure. 


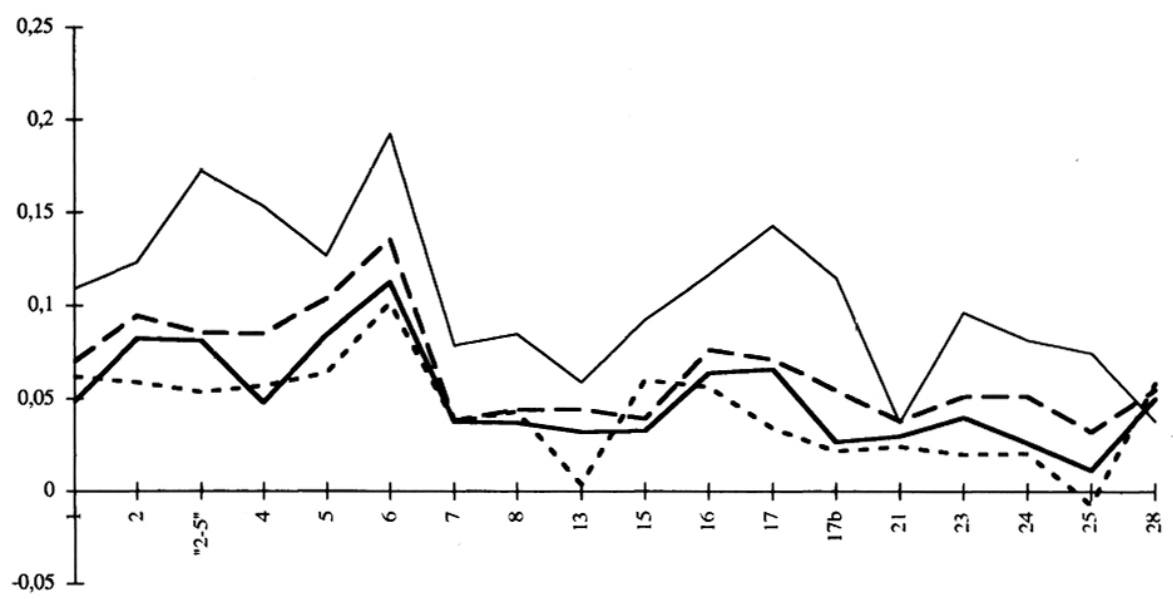

Przewalsky - - E.cab. actuels - - - - Saintes Moy.P.m.

Figure 8 : Comparaison de deux crânes de chevaux holocène. Diagramme établi selon la méthode de Simpson. Mesures V. Eisenmannet al. (1985) et P. Caillat (inédit). En référence (0) : les dimensions moyennes du crâne chez Equus hemionus onager d'après V. Eisenmann et al., 1985. Le trait fin indique pour les 3 crânes de chevaux du Pléistocène moyen la valeur "moyenne" de chaque mesure.

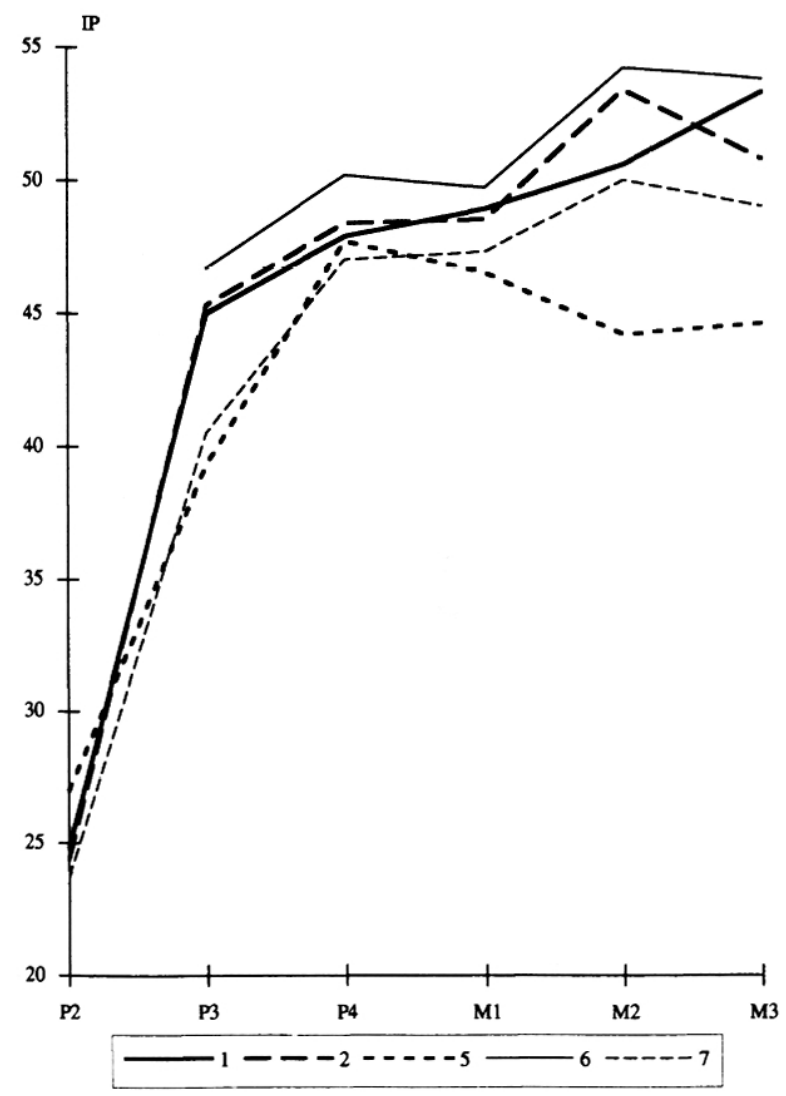

Figure 10 : Evolution de l'indice protoconique dans la série $\mathbf{P}^{2}, \mathbf{P}^{3}, \mathbf{P}^{4}, \mathbf{M}^{1}, \mathbf{M}^{2}, \mathbf{M}^{3}$ (chevaux du Pléistocène moyen). 1-Camp-de-Peyre, 2-Mosbach, 5-Tilloux, 6-Vergranne, 7-Lunel-Viel. 

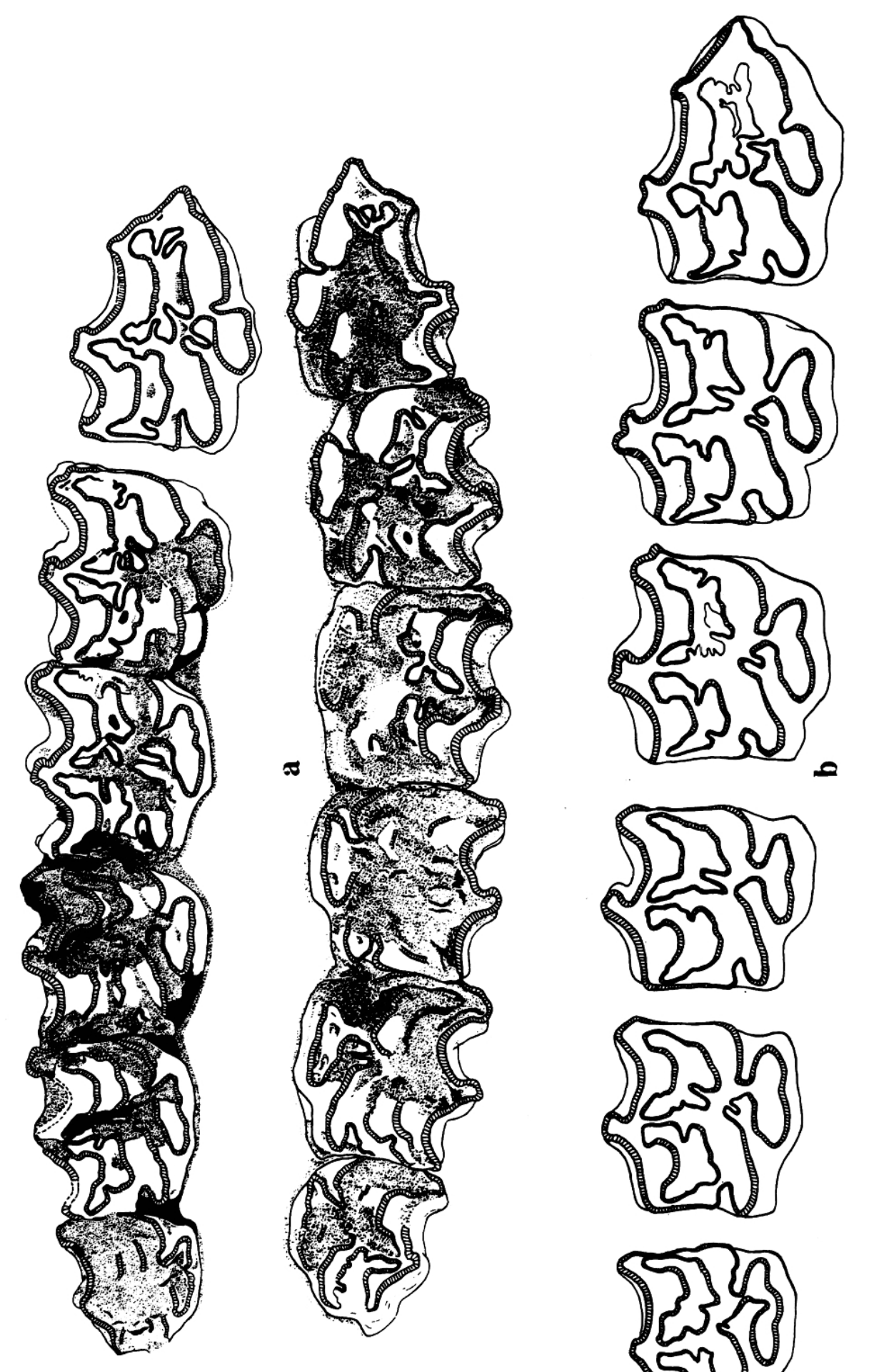

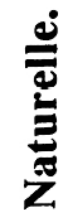

光

층

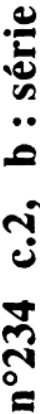
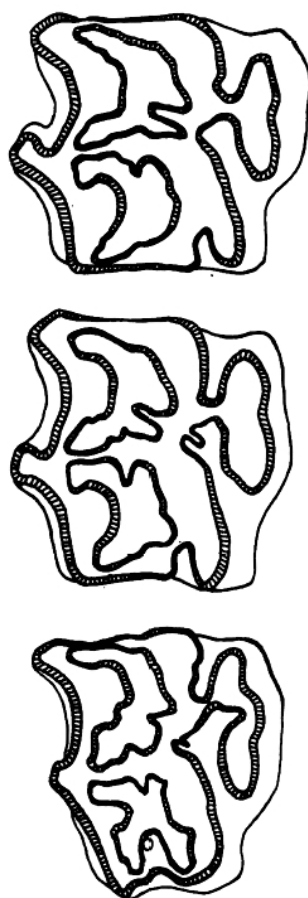

U 


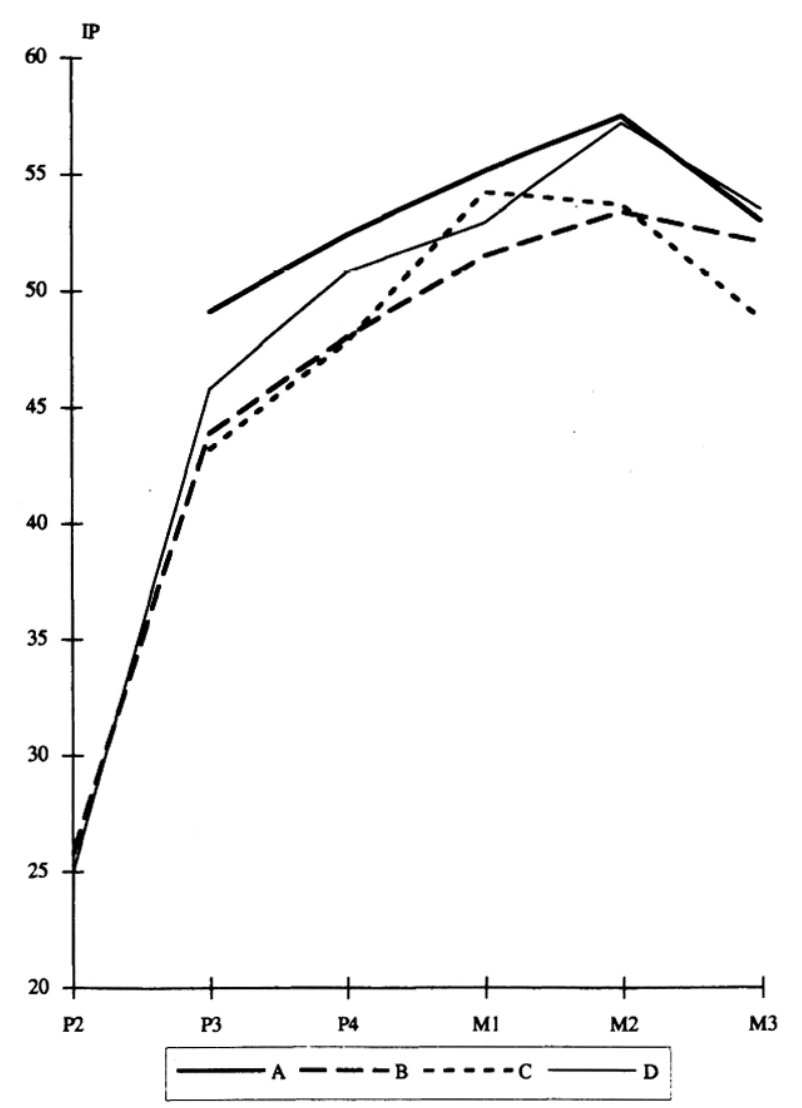

Figure 11 : Evolution de l'indice protoconique dans la série $\mathbf{P}^{2}, \mathbf{P}^{3}, \mathbf{P}^{4}, \mathbf{M}^{1}, \mathbf{M}^{2}, \mathbf{M}^{3}$ (chevaux du Pléistocène supérieur et de l'Holocène).

A-Abri Suard, B-Combe-Grenal, C-Jaurens, D-Solutré (Magdalénien).

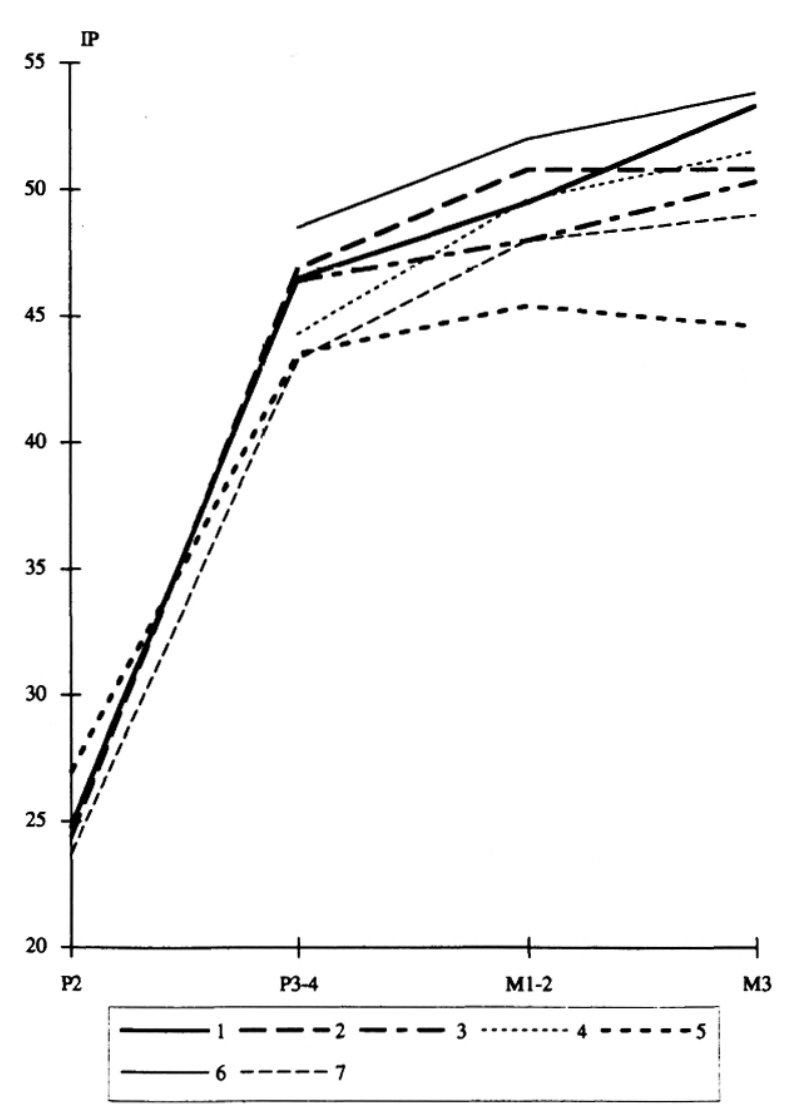

Figure 12 : Evolution de l'indice protoconique dans la série $\mathrm{P}^{2}, \mathrm{P}^{3-4}, \mathrm{M}^{1-2}, \mathrm{M}^{3}$ (chevaux du Pléistocène moyen). 1-Camp-de-Peyre, 2-Mosbach, 3-Montoussé, 4-La Micoque, 5-Tilloux, 6-Vergranne, 7-Lunel-Viel.

115 


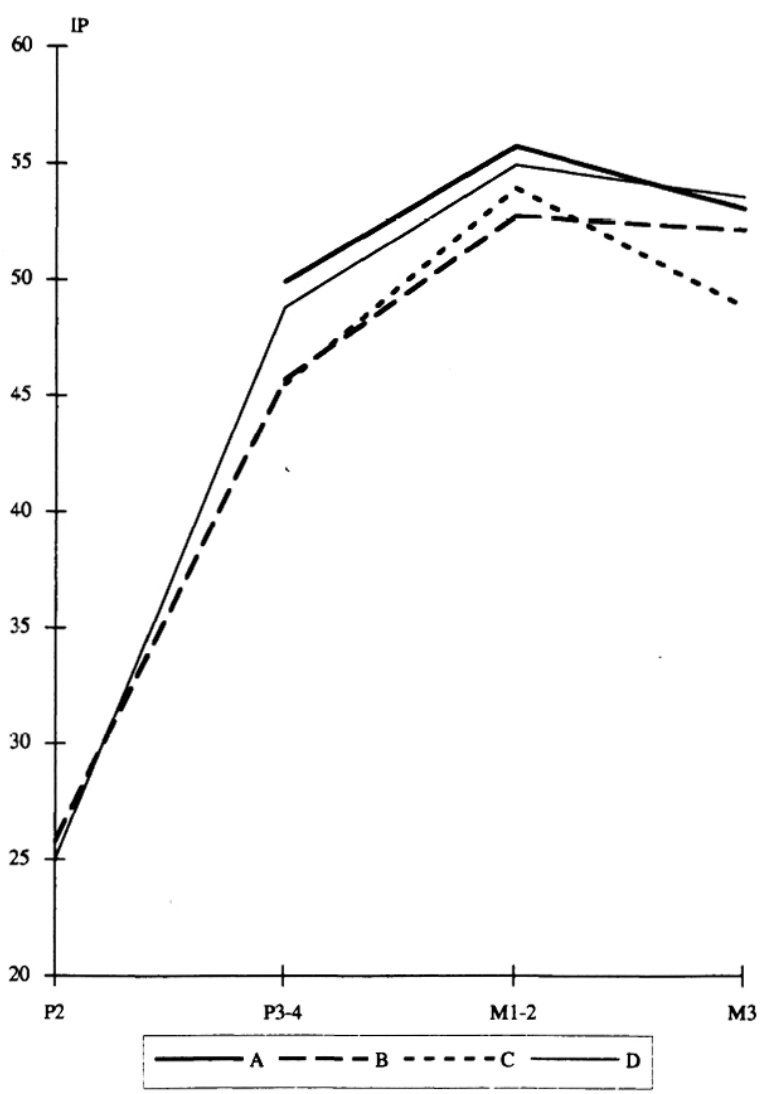

Figure 13 : Evolution de l'indice protoconique dans la série $\mathbf{P}^{2}, \mathbf{P}^{3-4}, \mathbf{M}^{1-2}, M^{3}$ (chevaux du Pléistocène supérieur). A-Abri Suard, B-Combe-Grenal, C-Jaurens, D-Solutré (Magdalénien).

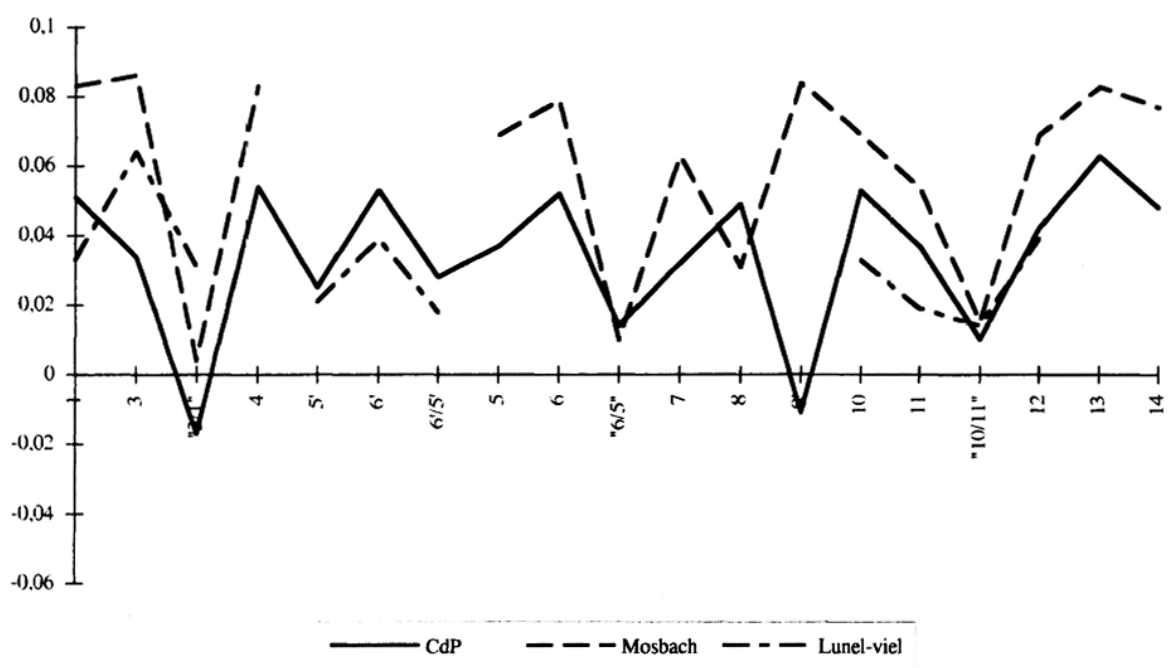

Figure 16 : Dimensions moyennes des métacarpiens III de chevaux du pléistocène moyen. Diagramme établi selon la méthode de Simpson. En référence (0) : les dimensions moyennes des métacarpiens III d'Equus caballus arcelini (Würm récent) d'après J.-L. Guadelli (1991). 

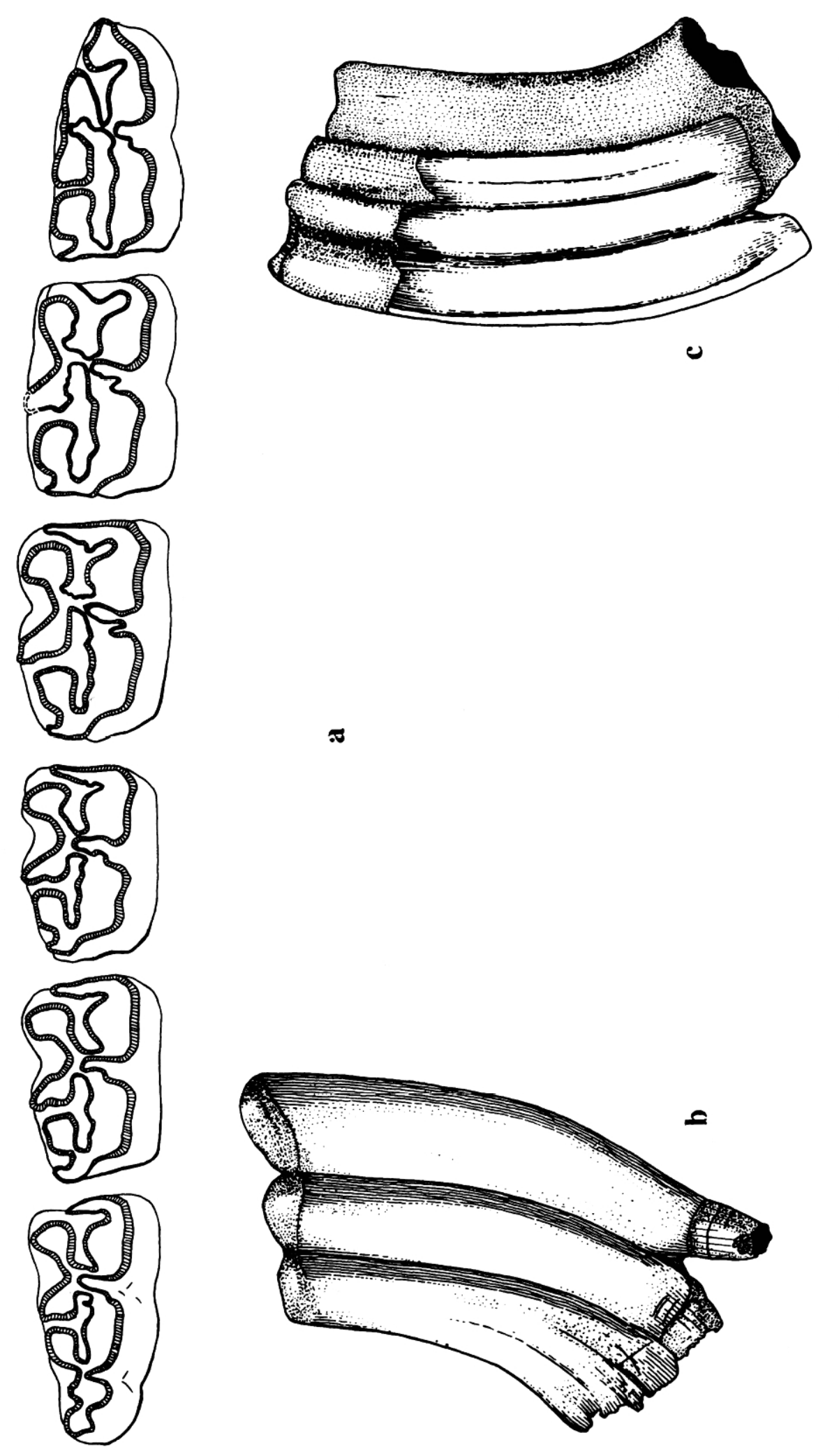

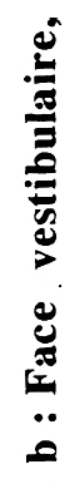

ิㅗㅀ

ن

ร

نَّ

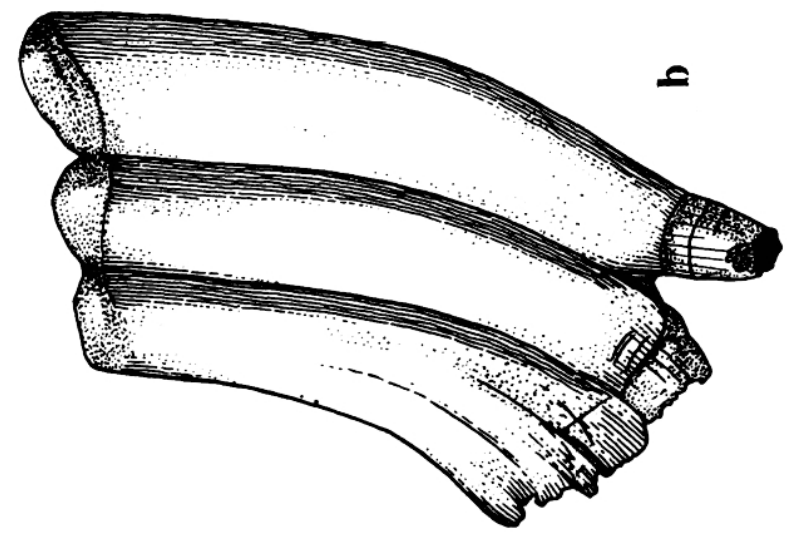

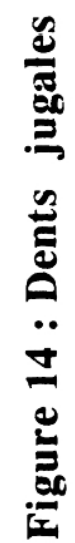




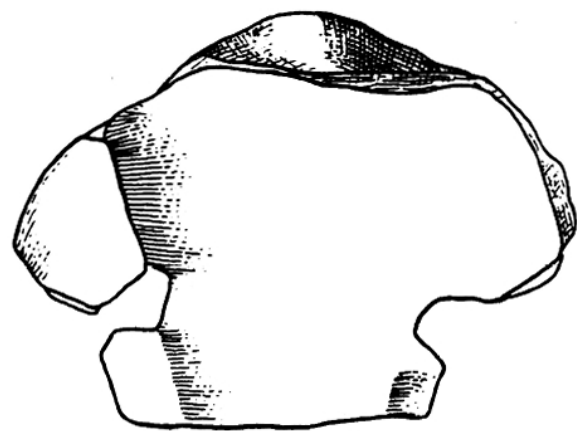

a

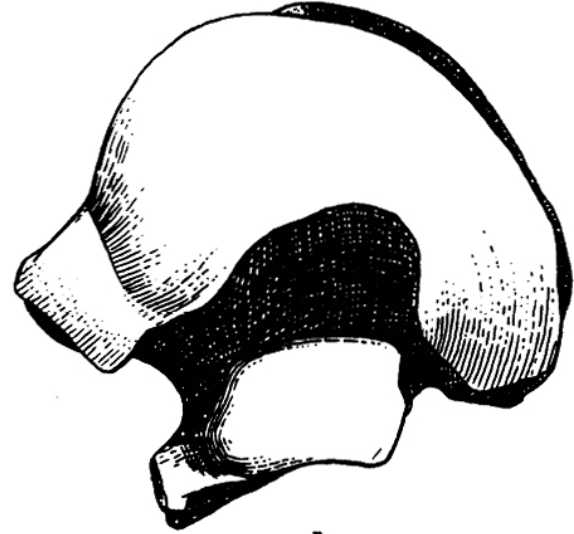

b

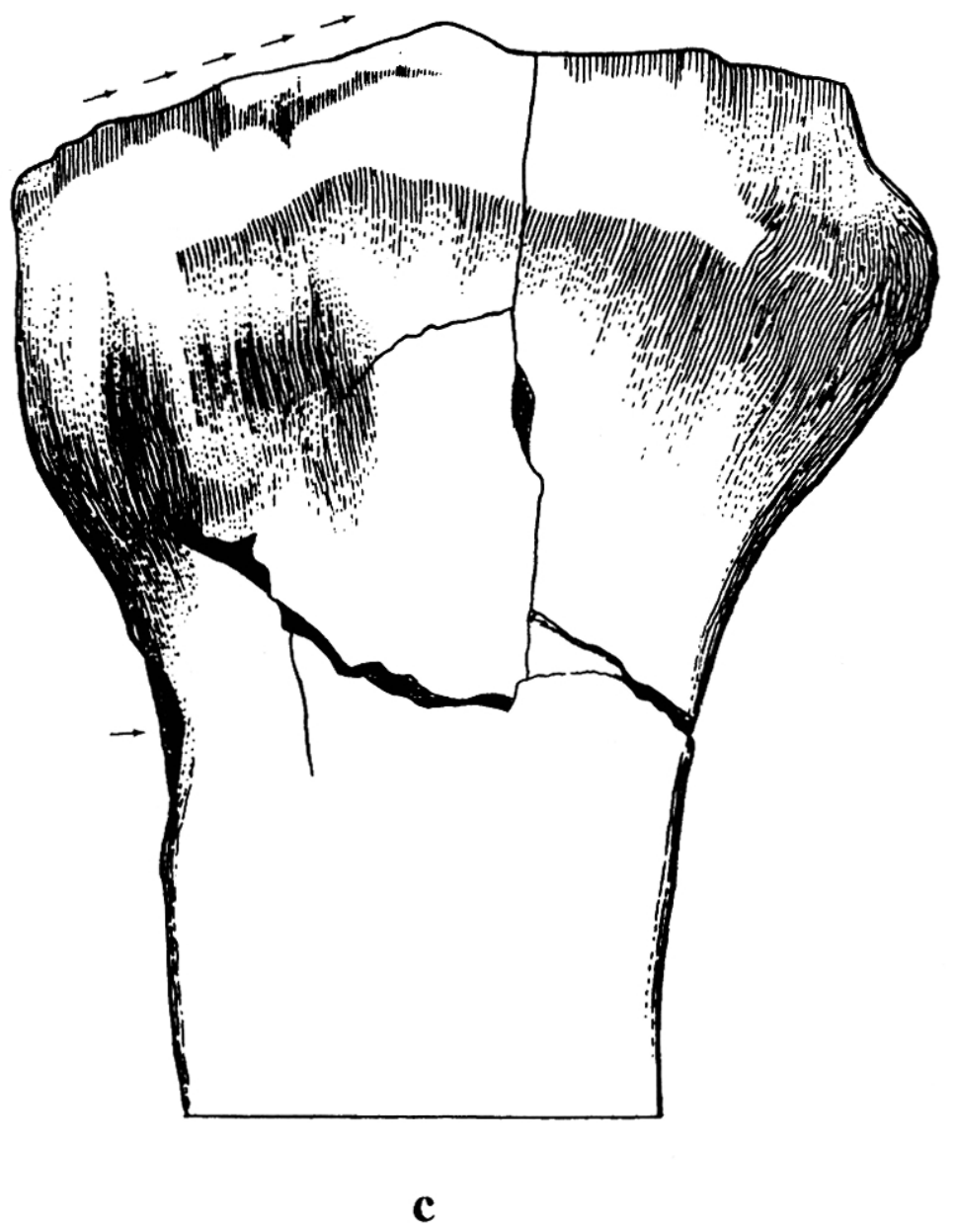

Figure 15 : a : Face articulaire proximale de métacarpien III gauche $\left(n^{\circ} 22\right)$, b : Face articulaire de métatarsien III gauche $\left(n^{\circ}\right)$ c : portion proximale de radius gauche $\left(n^{\circ} 25\right)$. Grandeur Naturelle. 


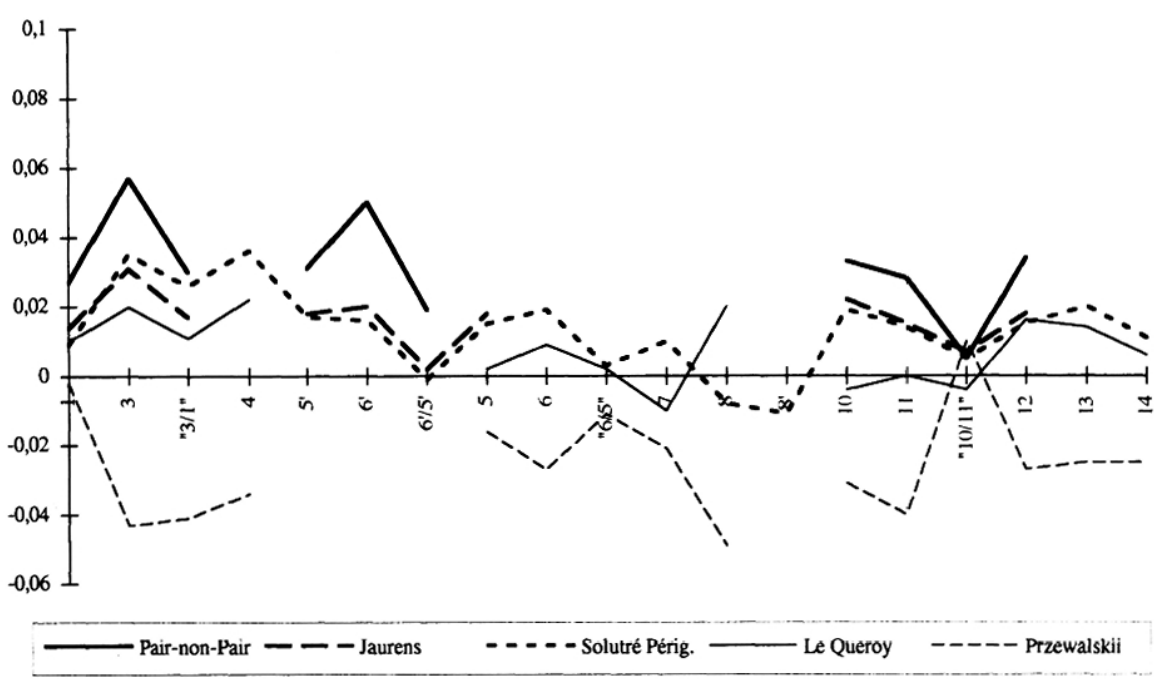

Figure 17 : Dimensions moyennes des métacarpiens III de chevaux du pléistocène supérieur et de I'holocène. Mesure C. Mourer-Chauviré (1980) et V. Eisenmann (1979). Diagramme établi selon la méthode de Simpson. En référence (0): les dimensions moyennes des métacarpiens III d'Equus caballus arcelini (Würm récent) d'après J.-L. Guadelli (1991).

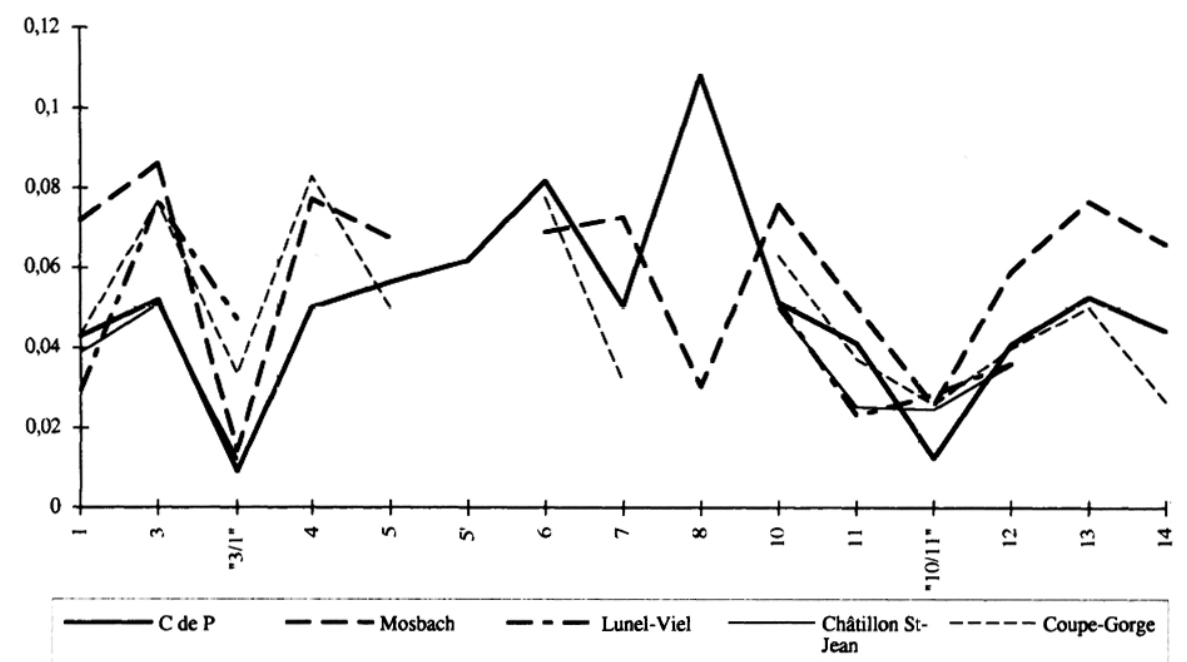

Figure 18 : Dimensions moyennes des métatarsiens III de chevaux du pléistocène moyen. Diagramme établi selon la méthode de Simpson. En référence (0): les dimensions moyennes des métatarsiens III d'Equus caballus arcelini (Würm récent) d'après J.-L. Guadelli (1991). 


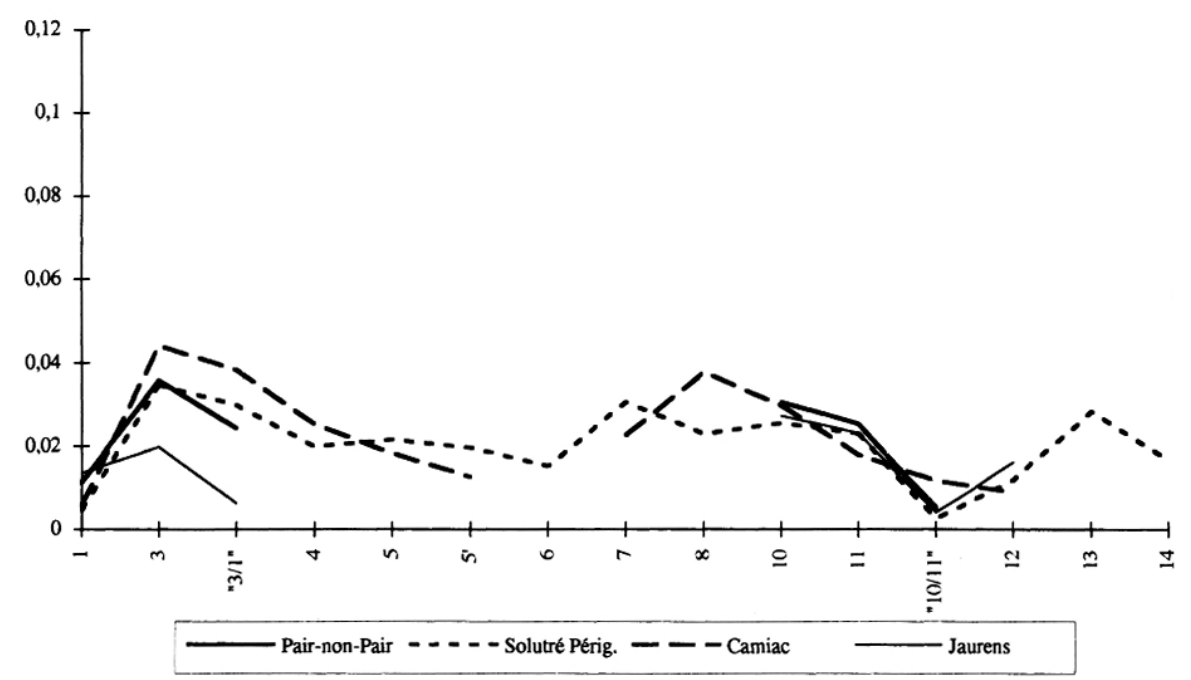

Figure 19 : Dimensions moyennes des métatarsiens III de chevaux du pléistocène supérieur et de l'holocène. Jaurens : mesures C. Mourer-Chauviré (1980). Diagramme établi selon la méthode de Simpson. En référence (0) : les dimensions moyennes des métatarsiens III d'Equus caballus arcelini (Würm récent) d'après J.-L. Guadelli (1991).

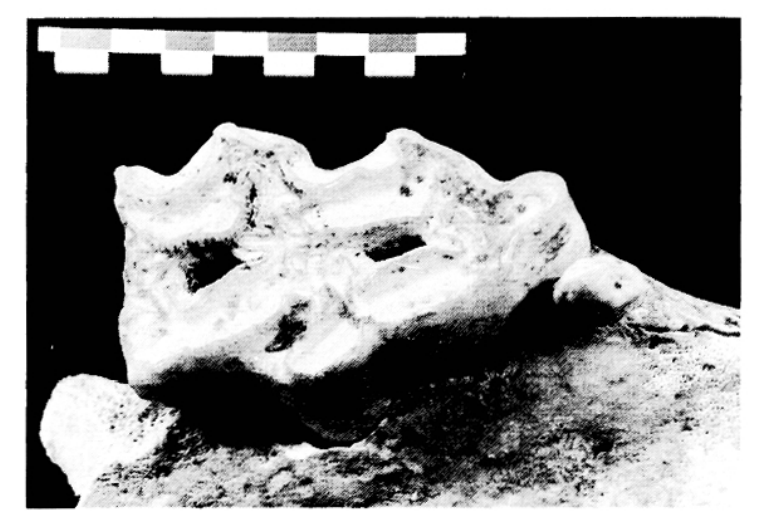

Planche I : Camp-de-Peyre. Equus mosbachensis campdepeyri Crâne Norma lateralis (c.2, $\left.\mathbf{n}^{\circ} 234\right)$. $1 / 2$ grandeur naturelle.

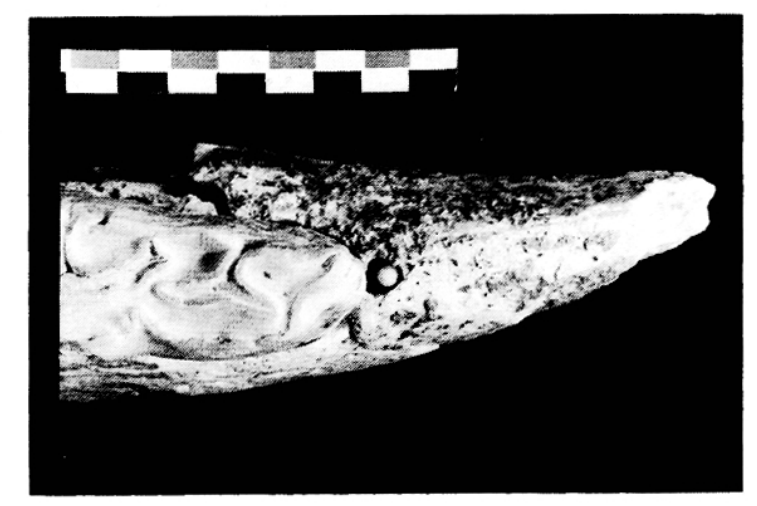

Planche II : Portion de maxillaire droit $\left(n^{\circ} 146\right.$ c.2) portant D(P)1-D4. Grandeur naturelle. 


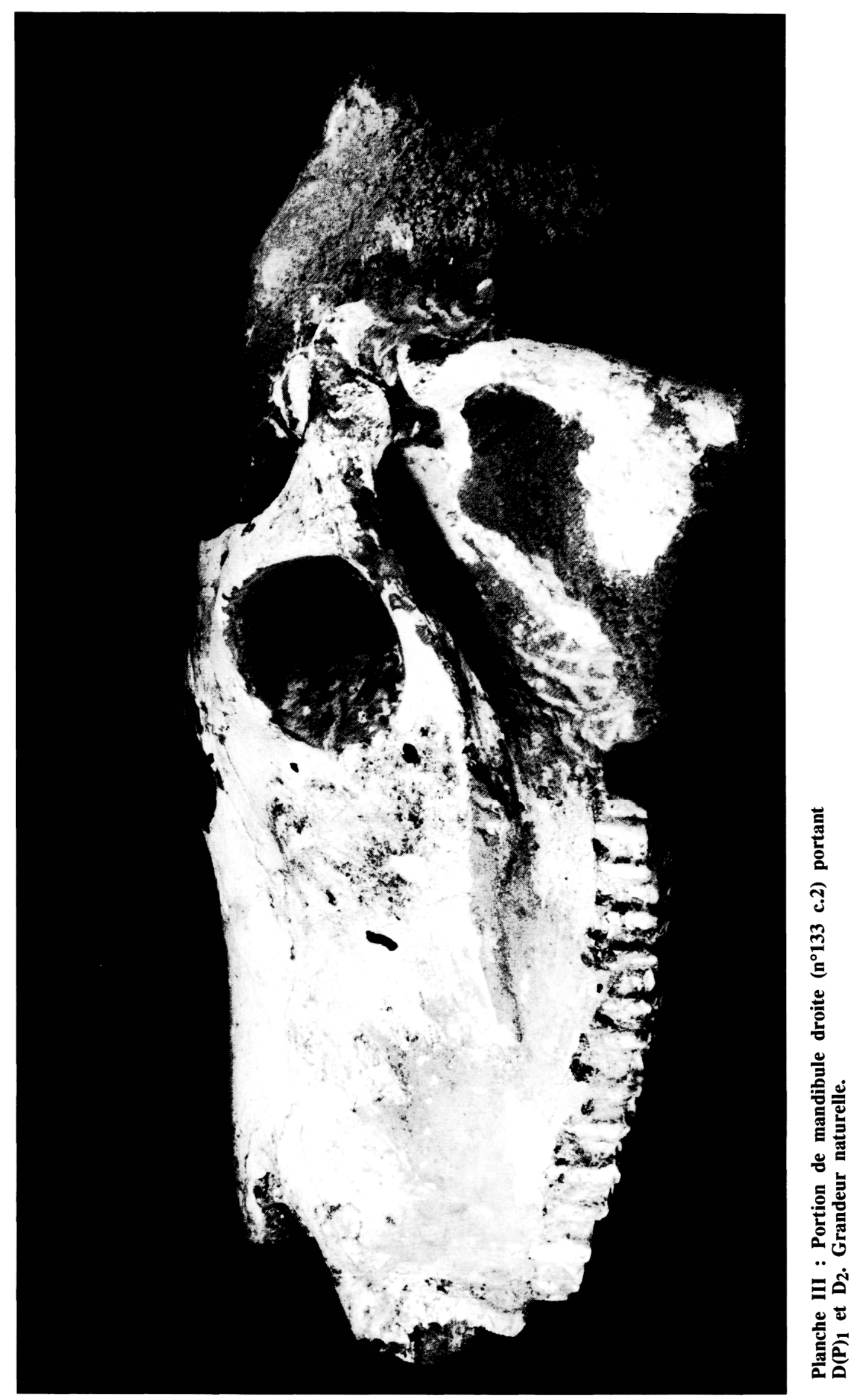

